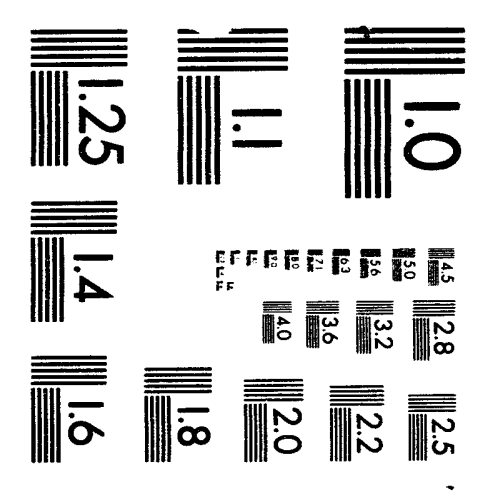

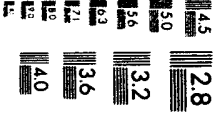



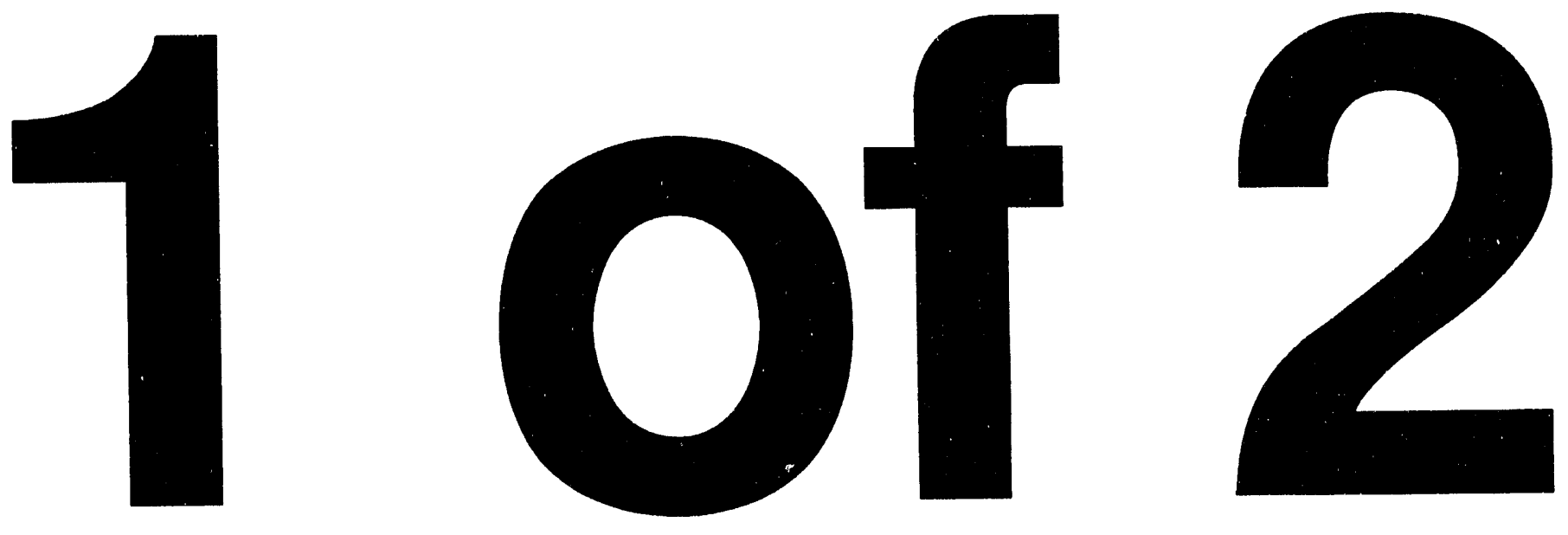


\title{
Theoretical Studies of Zirconium and Carbon Clusters with Molecular Dynamics Simulations
}

\author{
by Bulin Zhang
}

\author{
thesis submitted to Iowa State University \\ Ames Laboratory, U. S. DOE \\ Iowa State University \\ Ames, Iowa 50011
}

Date Transmitted: August 1993

\author{
Prepared for the U. S. Department of Energy \\ Under contract no. W-7405-eng-82.
}

\section{DISCLAIMER}

This report was prepared as an account of work sponsored by an agency of the United States This report was prepared as an account of work sponsored by an agency thereof, nor any of their employees, makes any warranty, express or implied, or assumes any legal liability or responsibility for the accuracy, completeness, or usefulness of any information, apparatus, product, or process disclosed, or represents that its use would not infringe privately owned rights. Reference herein to any specific commercial product, process, or service by trade name, trademark, manufacturer, or otherwise does not necessarily constitute or imply its endorsement, recommendation, or favoring by the United States Government or any agency thereof. The views and opinions of authors expressed herein do not necessarily state or reflect those of the United States Government or any agency thereof. 
TABLE OF CONTENTS

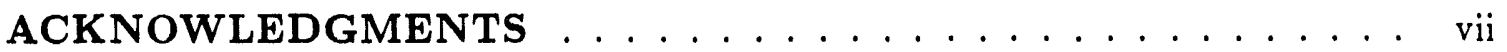

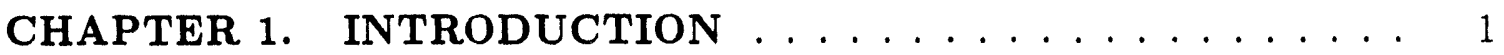

CHAPTER 2. EMBEDDED-ATOM METHOD POTENTIAL FOR

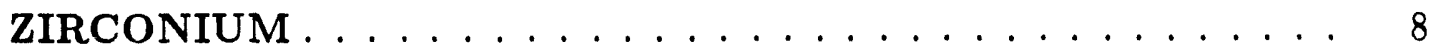

CHAPTER 3. MOLECULAR DYNAMICS SIMULATION . . . . 15

Molecular Dynamics . . . . . . . . . . . . . . . . 15

Constant Pressure and Constant Temperature Simulation . . . . . . . 18

Thermal Expansion and Melting . . . . . . . . . . . . 21

CHAPTER 4. ANOMALOUS PHONON BEHAVIOR AND PHASE

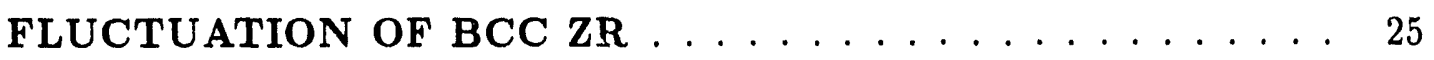

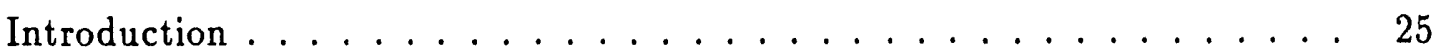

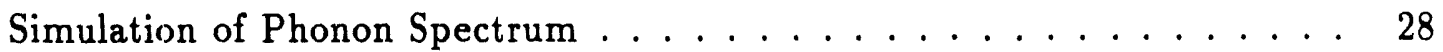

Anomalous Phonon Behavior and Phase Fluctuation at high temperature $b c c$ phase . . . . . . . . . . . . . . 30

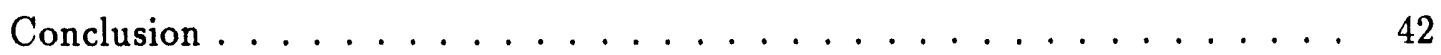

CHAPTER 5. TIGHT-BINDING MOLECULAR DYNAMICS . . 44

Concepts and Applications in Carbon System . . . . . . . . . . 44 
Implementation of Electronic Temperature . . . . . . . . . . . ts

CHAPTER 6. STRUCTURES AND STABILITIES OF FULLERENE

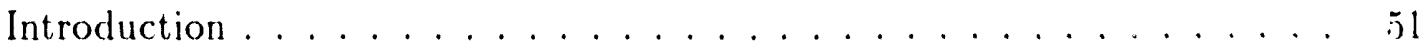

Ground-State Structure Search Scheme . . . . . . . . . . . . . 5t

Results and Discussion ................... . . . . . . . . .

Stabilities of Fullerenes . . . . . . . . . . . . . . . . 82

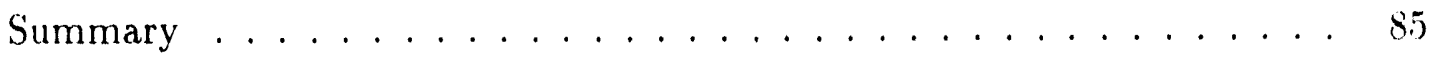

CHAPTER 7. THREE DIMENSIONAL CARBON NETWORKS $8 \&$

Introduction . . . . . . . . . . . . . . . . . . 88

Search Scheme . . . . . . . . . . . . . . . . 90

Structural, Electronic and Vibrational Properties . . . . . . . . . 91

Summary .......................... 96

CHAPTER 8. THERMAL DISINTEGRATION OF FULLERENES 99

Introduction . . . . . . . . . . . . . . . . . . . . 99

Details of Simulations . . . . . . . . . . . . . . . 100

Results and Discussion .................. 102

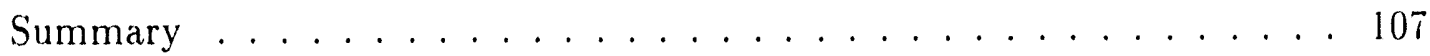

CHAPTER 9. COLLISIONS OF FULLERENES . . . . . . . . . . . 109

Introduction . . . . . . . . . . . . . . . . . 109

Simulations of Fullerene Collisions . . . . . . . . . . . . . 110

Results and Discussions .................. 111

Summary ......................... 123

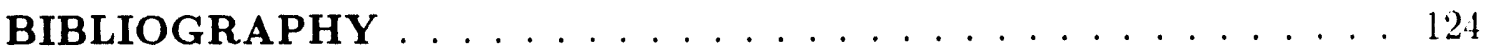




\section{LIST OF TABLES}

Table 2.1: Parameters used to calculate the atomic density of $\mathrm{Zr}$. . . . 11

Table 2.2: $\quad$ Parameters of potentials EAM1 and EAM2 for $\mathrm{Zr}$. . . . . . 12

Table 2.3: Quantities used for determination of the functions $F(\rho)$ and $\mathrm{Z}(\mathrm{r})$ for $\mathrm{Zr}$ and their fitted values. . . . . . . . . . 12

Table 6.1: Enumeration of distinct isolated-pentagon $\mathrm{C}_{n}$ fullerene isomers. 53

Table 6.2: $\quad$ Structural and electronic properties of carbon clusters. . . . . 57

Table 7.1: Binding energies of three-dimensional carbon networks. . . . 95

Table 7.2: $\quad$ Gaps and densities of three-dimensional carbon networks. . . 95

Table 9.1: Fusion energies of fullerene collisions. . . . . . . . . 116

Table 9.2: Fragmentation energies of fullerene collisions. . . . . . . . 116 


\section{LIST OF FIGURES}

Figure 2.1: Phonon dispersion curves of $b c c$ and $h c p \mathrm{Zr}$ for EAM1. . . 13

Figure 2.2: $\quad$ Phonon dispersion curves of $b c c$ and $h c p \mathrm{Zr}$ for EAM2. . . . 14

Figure 3.1: Thermal expansion of Zr. . . . . . . . . . . . . . . 23

Figure 3.2: Melting of $\mathrm{Zr} . \ldots \ldots \ldots \ldots$

Figure 4.1: Geometric relations between $b c c, h c p$ and $\omega . \ldots . \ldots 2 \tau$

Figure 4.2: The phonon spectra of $h c p$ Zr. . . . . . . . . . . . . 31

Figure 4.3: The radial distribution functions of $b c c$ and $h c p \mathrm{Zr} \ldots \ldots 33$

Figure 4.4: Dynamical structure factors at selected $Q$ points of high tem-

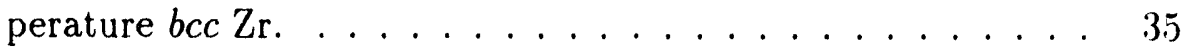

Figure 4.5: Contour plots of the integrated scattering intensity . . . . . 36

Figure 4.6: Time evolutions of $S(\mathrm{t})$ and $N_{h c p}(\mathrm{t}) \ldots \ldots \ldots \ldots$

Figure 4.7: The snapshot picture of atomic configuration . . . . . . . 39

Figure 4.8: Geometric structure factors of strong and weak $\omega$ points along the $b c c$ to $h c p$ transformation path. . . . . . . . . 42

Figure 6.1: Line drawings of carbon fullerenes. . . . . . . . . . . . . . . 60

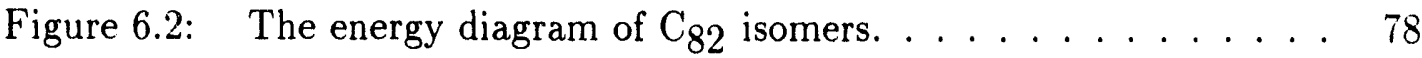

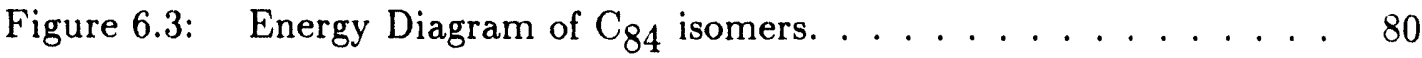


Figure 6.4: Ball and stick models of $\mathrm{C}_{84}$ isomers $\ldots \ldots \ldots \ldots 1$

Figure 6.5: The heat of formation of carbon clusters. . . . . . . . . 8.3

Figure 6.6: The fragmentation energy and HOMO-LUMO energy separa-

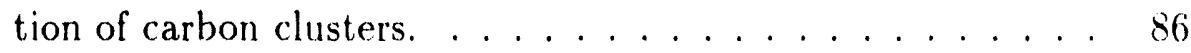

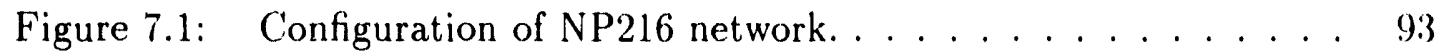

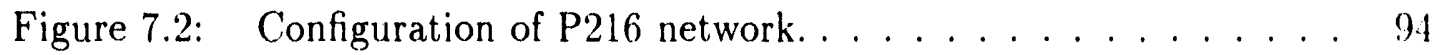

Figure 7.3: Vibrational spectra of NP216 network, graphite and diamond. 97

Figure 8.1: The potential energy of $\mathrm{C}_{60}$ as function of temperature. . . 101

Figure 8.2: Fragmentation temperatures of carbon fullerenes. . . . . . . 104

Figure 8.3: Fragmentation process of $\mathrm{C}_{60} \ldots \ldots \ldots \ldots \ldots$

Figure 8.4: Temperature dependence of HOMO-LUMO gap for $\mathrm{C}_{60} \ldots 108$

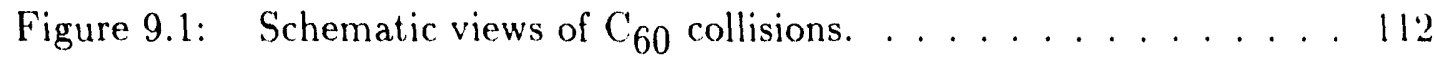

Figure 9.2: Illistrations of three different collision stages. . . . . . . 114

Figure 9.3: Energy transfer ratio and final temperature of fullerene collisions. . . . . . . . . . . . . . . . 117

Figure 9.4: Time evolution of kinetic energy, potential energy and center of mass separation in fullerene collision. . . . . . . . . 120

Figure 9.5: Products of collisions between two $\mathrm{C}_{60} \ldots \ldots \ldots 122$ 


\section{ACKNOWLEDGMENTS}

I wish to express my sincere thanks to my major Professor K. M. Ho for his kind and enthusiastic guidance. I also wish to thank Drs. C. T. Chan and C. Z. Wang for their patient help in every detail of this work. I am grateful for Professor B. N. Harmon for his suggestions and encouragements. I am also grateful for Dr. D. E. Turner, Professor C. Stassis, Ms. Y. Y. Ye, and Dr. J. R. Morris for their help and stimulating discussions.

I thank my wife, Hui Wang, for her understanding and support throughout our marriage. My thanks extend to my parents back China for their constant support in my whole life.

This work was performed at Ames Laboratory under contract no. W-7405-eng82 with the U. S. Department of Energy. The United States government has assigned the DOE Report number IS-T 1662 to this dissertation. I acknowledge the Sandia National Laboratories and Scalable Computing Laboratory at Iowa State university for providing time on their n-CUBE. Part of this work is performed on the Intel/IP860 machine at Oak Ridge National Laboratories. 


\section{CHAPTER 1. INTRODUCTION}

As a model system for martensitic phase transitions (MT), $\mathrm{Z}_{\mathbf{r}}$ and $\mathrm{Zr}$ based alloys have received a lot of attention from both experimentalists and theorists $[1,2$, $3,4,5,6,7,11,12,13,14,15,16,17,18]$. Zr has a $h c p$ lattice ( $\alpha$ phase) at room temperature and a $b c c$ lattice ( $\beta$ phase) above $1136 \mathrm{~K}$. Another hexagonal crystalline structure, the $\omega$ phase, appears in pure $\mathrm{Zr}$ under high pressure or upon quenching in some $\mathrm{Z}_{\mathrm{r}}$ alloys [1]. The high temperature bcc lattice exhibits intrinsic instability toward $h c p$ and $\omega$ structures through various anomalous phonon behavior.

Inelastic neutron measurements on bcc $\mathrm{Zr}$ and $\mathrm{Zr}$ alloys indicate that the longitudinal $[\xi \xi \xi]$ phonon at $\frac{2}{3}(1,1,1)\left(\omega\right.$ point) as well as the $\mathrm{T}_{1}$ phonon at $\frac{1}{2}(1,1,0)$ ( $N$ point) have very low frequencies and strong quasi-elastic intensities $[19,20,21]$. Another most intriguing feature is that the measured phonon spectrum and the quasielastic scattering exhibit a strong asymmetry around the $(2,2,2)$ bcc Bragg peak along the $[11 \overline{2}]$ direction $[4,5,11]$. This violates the $b c c$ symmetry which would hold for a normal crystal. It is very interesting that this anomalous phonon behavior appears to be generic for a wide range of systems in which the $b c c$ phase transforms to another structure at low temperatures $[19,20,21,22,23]$.

A low frequency $\mathrm{T}_{1}$ phonon at the $N$ point is a precursor of the $b c c-h c p$ martensitic phase transition, while the sharp dip in the longitudinal phonon dispersion in 
the $[\xi \xi \xi]$ direction at the $\omega$ point is associated with bcc to $\omega$ phase transition. It was generally accepted that the anomalous phonon behavior observed in the bcc phase at high temperature is associated with the bcc to $\omega$ phase transition since the quasielastic peaks are observed at wavevector positions equivalent to the $\omega$ point in $\mathrm{Zr}$ alloys. However, recent work by Heiming et al. [20] on pure $\mathrm{Zr}$ crystals raised the possibility that $b c c-h c p$ phase fluctuations may also be important.

On the other hand, the first-principles calculations $[12,14]$ found that energy dependence on displacement of the longitudinal $\omega$ point phonon is extraordinarily anharmonic, while the $b c c$ phase is unstable against the displacement of the $T_{1} N$ point phonon. The stability of the $b c c$ phase against the hcp phase is achieved by strong phonon-phonon coupling [14]. But these first-principles calculations are all performed at zero temperature and the anharmonicity is included only through perturbation theory. There are extensive efforts $[13,15,16,17,18]$ to study the relations between these soft phonons and phase transitions. However, a complete picture, which can account for all the above experimental observations, is still lacking.

In the first part of this dissertation, we employ the embedded-atom method (EAM) $[24]$ to study anomalous phonon behavior and phase fluctuation phenomena in high temperature bcc $\mathrm{Zr}$. The EAM potential is composed of a many-body embedding function and a two-body Coulomb-type interaction. This kind of potential model is far superior to two-body potentials (e.g. Lennard-Jones potential) but is still simple enough to handle large systems. This method includes the essential physics of metal systems with closed-packed structure and it has been successfully used to study metal surfaces, liquids, phonons and alloy systems $[25,26,27,28]$. This kind of potential is particularly popular for molecular dynamics simulations since the 
program can be run efficiently on massively parallel computers. Studies with large systems up to a million atoms or long simulation time up to microseconds have been reported [29]. In our molecular dynamic simulations, we studied both the low temperature $h c p$ phase and the high temperature $b c c$ phase of $\mathrm{Zr}$. At high temperatures, the system is highly anharmonic and the usual assumption that the scattering crosssections are dominated by one-phonon processes no longer holds. The commonly used experimental interpretations of zone dependence of phonon shape as well as the usual approximations employed in the theoretical calculations of phonon spectra need to be reexamined.

Our simulations reproduced all the anomalous phonon behavior observed in experiments for high temperature $b c c \mathrm{Zr}$. From the analysis of the real space trajectories of atoms, we found that the phase fluctuations between $b c c$ and $h c p$ play the most important role. The asymmetry in neutron scattering measurement is related to the incompleteness of the $b c c$ to $h c p$ transition.

The second part of this dissertation involves a study of carbon clusters. Carbon is a kind of natural backbone: the all-important element that anchors the molecules of everything from crude oil to DNA. Carbon clusters have been a subject of interest for more than a decade. The first observation of carbon clusters was made by laser vaporization of graphite in 1984 [30]. In the mass spectrum, the clusters start from high 30 s and extend out well beyond $C_{100}$ and this mass distribution contains only even-number clusters. This is rather remarkable, since nothing like this even-number distribution had ever been seen for any other element [31]. Shortly after that, Kroto et al. [32] came up with the brilliant idea that this mass distribution can be explained if the carbon clusters possess a hollow spherical structure. For carbon networks with 
coordination number of three, only even-numbered atoms can enclosed themselves without the presence of dangling bonds which are energetically very unfavourable. This new family of carbon clusters are called fullerenes because they resemble the geodesic domes designed by the architect R. Buckminster Fuller in 1983.

Among all the fullerenes, $\mathrm{C}_{60}$ is the most abundant in the mass spectrum [32]. The structure proposed for $\mathrm{C}_{60}$ is a truncated icosahadron, resembling a soccer ball. All the carbon atoms are located at the 60 vertices of a truncated icosahedron, forming 12 five-membered rings and 20 six-membered rings. In the $\mathrm{C}_{60}$ structure, all the carbon atoms are equivalent from symmetry. This structure for $\mathrm{C}_{60}$ was not confirmed until the breakthrough [33] in 1990 when it was discovered that macroscopic quantities of $\mathrm{C}_{60}$ can be isolated from the soot formed when graphite rods are burned in an inert helium atmosphere. Detailed NMR [34] and infrared spectrum [35] obtained on the $\mathrm{C}_{60}$ samples unambiguously determined that the structure of the $\mathrm{C}_{60}$ molecr:le is indeed the proposed truncated icosahedral cage. Subsequently, numerous fascinating properties of fullerene and fullerene derivatives, such as superconductivity [36], ferromagnetism [37] and encapsulation of metal atoms [38] have been reported. The emergence of the carbon fullerene family led to a rapid surge in interest of scientists from various areas and $\mathrm{C}_{60}$ is regarded as the molecule of the decade.

To study the behavior of carbon clusters with molecular dynamics, we need an accurate potential describing the interatomic interactions among carbon atoms. Carbon is an unique element in the sense that it can form structures with coordination number three and four, corresponding to the two dimensional graphite sheets (coordination number three) and the three dimensional diamond (coordination number four). Although there are several classical potential models for carbon $[39,40]$, they 
are not satisfactory for the description of small carbon clusters because of the importance of quantum-mechanical electronic structure effects in the carbon system. In this dissertation, we employ an empirical tight-binding (TB) potential model [41] which has several advantages compared with classical potentials and ab-initio calculations. First, the inclusion of the basic features of the electronic structures in the potential allows an natural and accurate description of the directional covalent bonding between carbon atoms. Secondly, only a small basis set is used in the empirical TB model, making it computationally much faster than ab-initio calculations.

Besides $\mathrm{C}_{60}$, larger fullerene clusters can also be isolated from the soot $[42,43$, $44,45,46]$, but the structures of many of these large fullerenes are still not clear. Characterization of the structures of large fullerenes is difficult both in theory and experiment due to the large number of possible structures as the size of the cluster increases. In this dissertation, we will present a systematic study of structures of fullerenes ranging from $\mathrm{C}_{20}$ to $\mathrm{C}_{100}$ by introducing a novel scheme [47]. Using our new scheme, we not only reproduce all known fullerene structures but also successfully predicted several other fullerene structures which were confirmed by experiments $[48,49,50]$. By utilizing the tight-binding molecular-dynamic (TBMD) simulation, we also studied the dynamical behavior of fullerenes: vibrations [51], thermal disintegration [52] of individual clusters as well as collisions between fullerenes [53]. If the beauty of carbon fullerene is not enough, people found that carbon can also form tubules [54] and even speculated that they can form three-dimensional graphite-like networks $[117,118,119]$. By extending our fullerene structure searching scheme, we performed a search for the ground-state structure of three dimensional carbon network [58]. We found the most stable structure people ever proposed for simple cubic 
based networks. From the difference of this new form of carbon and graphite in the electronic and vibrational properties, we propose an experimental probe to identify these novel three-dimensional carbon networks.

In Chapter 2, the fitting procedure of EAM potential will be described and some relevant physical properties of $\mathrm{Zr}$ calculated from this potential model are presented.

In Chapter 3, the basic concepts of molecular dynamics are reviewed.

In Chapter 4, the details of molecular dynamics simulation of high temperature bcc $\mathrm{Zr}$ is described. Massively parallel computers are utilized to simulate large unit cells for long times. Our simulation can well reproduce all experimental observations and the origin of these anomalous phonons is proposed based on evidences extracted our simulations.

In Chapter 5, the theoretical background of the empirical tight-binding model is reviewed. The details of tight-binding molecular dynamics simulation and the implementation of electronic temperature is described.

In Chapter 6, the fullerene structures search (FSS) scheme is introduced. The results of our search along with the discussions of their implications are presented. Some of our theoretical predictions have already been confirmed by later experimental results.

In Chapter 7, a topological overview of carbon networks is presented. By extending FSS scheme, we search the ground-state three dimensional carbon networks. The similarities and differences in electronic structures and vibrational spectra between these novel carbon form and graphite are discussed.

In Chapter 8, we simulate the thermal disintegration of carbon clusters ranging from $\mathrm{C}_{20}$ to $\mathrm{C}_{90}$. The process of thermal disintegration of fullerenes is studied and 
the general trend of the disintegration temperature as a function of cluster size is discussed.

In Chapter 9, we simulated the collisions between carbon clusters. The collisions between $\mathrm{C}_{60}$ molecules are carefully studied under different conditions: cluster temperature, collision energies and orientations. To get statistical meaningful results, we used the parallel computers to simulate different trajectories simultaneously. 


\section{CHAPTER 2. EMBEDDED-ATOM METHOD POTENTIAL FOR ZIRCONIUM}

The theoretical investigation of the structural and dynamical properties of complex metallic systems, until recently, mostly relied on the use of interatomic potentials. The pair-potential scheme is the simplest form among the interatomic potential models, where the cohesive energy of a solid is given by a sum over pair bonds, plus a volume-dependent energy (one body interaction). Using pseudopotential theory, a perturbation series can be set up which expresses the interaction of atoms in jellium in terms of a one-body interaction as well as the two-body and higher interactions. The use of interatomic pair potentials suffers from the neglect of many-body interactions, which obviously play an important role in many physical phenomena. For example, two impurities in a metal interact through the host via a many-body interaction. In 1984, Daw and Baskes proposed a new framework for calculating the energetics of metals, which is called the embedded-atom method (EAM) [24]. In this approach, the energy of the metal is viewed as the energy to embed an atom into the local electron density contributed from the neighbor atoms. The remaining part of the total energy is expressed by a repulsive pair potential in the form of screened 
electrostatic interaction. The ansatz that they used is

$$
E_{c o h}=\sum_{i} F_{i}\left[\sum_{j(j \neq i)} \rho_{j}^{a}\left(R_{i j}\right)\right]+\frac{1}{2} \sum_{i, j(j \neq i)} \frac{Z\left(R_{i j}\right) Z\left(R_{i j}\right)}{R_{i j}}
$$

where $F$ is the embedded energy, $\rho^{a}$ is the spherically averaged atomic density, and $\mathrm{Z}\left(\mathrm{R}_{i j}\right)$ is an effective charge. The background density of each atom in Equation 2.1 is determined by evaluating at its nucleus the superposition of atomic density tails from the other atoms. Equation 2.1 combines the computational simplicity needed for complex systems with the physical inclusion of many-body effects. This method has been applied successfully to such problems as surfaces [25], liquid metals [26], phonons [27], and alloys [28],

The parameters and the formulism for calculating the atomic charge density are given in Table 2.1. The embedded function $F(\rho)$ and effective charge $Z(\mathrm{r})$ are determined from a cubic spline with 5 knots. The $Z(r)$ is set to be the atomic number of $\mathrm{Zr}$ at $\mathrm{r}=0$ and vanish at the cut off radius. Constraints were imposed upon $F$ to have a single minimum and $F$ is linear at higher densities. We chose $\rho / \bar{\rho}=2.5$ as a point when $F=0$, where $\bar{\rho}$ is the atomic density at equilibrium volume. In addition, to make the zero of energy correspond to neutral atoms separated to infinity, the function was constrained to go to zero at vanishing density. So the adjustable parameters here are the function values at three knots for both $F$ and $Z$. These parameters are fitted to the lattice constant, elastic constants and cohesive energy. The value at the spline knots as well as the fitting results are tabulated in Table 2.2 and Table 2.3. In our potential models, the cutoff radius is $6.25 \AA$ which is substantially larger than that used in the early EAM potential models. This is because the cohesive energies of $h c p$ and $b c c$ are so close that long range forces become important to differentiate them. 
By varying the positions of the cubic spline knots, we obtained two potential models, EAM1 and EAM2.

To further test the reliability of our potential models, we calculated the phonon dispersions along the high symmetry directions for both $h c p$ and $b c c$ structures in the quasiharmonic approximation. As plotted in Fig. 2.1 and Fig. 2.2 for two potential models respectively, the agreement with experimental measurements is satisfactory. The biggest discrepancy occurs at the $\Gamma$ point for the optical phonon mode of the $h c p$ phase. This anomalous phonon behavior is attributed to the detailed band structure near the Fermi level [61]. This anomalous property can not be accounted for by our classical interatomic potential.

The major difference between the phonon dispersions calculated from these two potential models is that the $\mathrm{T}_{1}$ phonon at the $N$ point is totally soft for EAM2. This means that the $b c c$ structure is no longer stable at zero temperature in potential EAM2 while the bcc lattice is still a metastable state in potential EAM1. The importance of this point will be discussed in Chapter 4 .

In the following chapters, all the results are obtained from potential EAM1 if they are not specified. 
Table 2.1: Parameters used to calculate the atomic density of $\mathrm{Zr}$. The atomic densities were taken from calculations by Clementi et al. [59]. The total density is given by $\rho^{a}(\mathrm{r})=\mathrm{N}_{s} \rho_{s}^{a}(\mathrm{r})+\mathrm{N}_{d} \rho_{d}^{a}(\mathrm{r})$, where $\mathrm{N}_{\mathcal{s}}^{Z r}=2$ and $\mathrm{N}_{d}^{Z r}=2$. The spherically averaged $\mathrm{s}$ - and d-like densities are computed by

$$
\begin{aligned}
& \rho_{s}^{a}=\left|\sum_{i} C_{i} R_{i}(r)\right|^{2} / 4 \pi \\
& R_{i}(r)=\frac{(2 \xi)^{\left(n_{i}+1 / 2\right)}}{\left[\left(2 n_{i}\right) !\right]^{1 / 2}} r^{n_{i}-1} e^{-\xi_{i} r}
\end{aligned}
$$

and the expression for $\rho_{d}^{a}$ is the same. The $\xi$ 's below are given in $\dot{A}^{-1}$.

\begin{tabular}{cccc}
\hline $\mathrm{i}$ & $\mathrm{n}_{i}$ & $\xi_{i}$ & $\mathrm{C}_{i}$ \\
\hline $5 \mathrm{~s}$ & & & \\
1 & 1 & 41.14930 & -0.00006 \\
2 & 1 & 28.22350 & 0.02115 \\
3 & 2 & 18.61520 & 0.07698 \\
4 & 2 & 17.00340 & -0.14888 \\
5 & 3 & 10.81480 & 0.01439 \\
6 & 3 & 7.82175 & 0.15025 \\
7 & 4 & 4.45263 & -0.25537 \\
8 & 4 & 3.03088 & -0.06341 \\
9 & 5 & 1.79574 & 0.55594 \\
10 & 5 & 1.04278 & 0.56329 \\
$4 \mathrm{~d}$ & & & -0.07279 \\
1 & 3 & 12.87330 & -0.17958 \\
2 & 3 & 6.90015 & 0.40742 \\
3 & 4 & 3.63923 & 0.72844 \\
4 & 4 & 1.80383 & \\
\hline \hline
\end{tabular}


Table 2.2: Parameters of potentials EAM1 and EAM2 for $\mathrm{Zr}$. The positions of the spline knots and the values at the knots are given. Distances are given in $\stackrel{A}{A}$, density in $\AA^{-3}$, charge in a.u., and energy in eV.

\begin{tabular}{cccccc}
\hline \hline EAM1 & \multicolumn{5}{c}{ EAM2 } \\
\hline $\mathrm{r}$ & $\mathrm{Z}(\mathrm{r})$ & $\mathrm{Z}^{\prime}(\mathrm{r})$ & $\mathrm{r}$ & $\mathrm{Z}(\mathrm{r})$ & $\mathrm{Z}^{\prime}(\mathrm{r})$ \\
\hline 0.0 & 40.0 & 0.0 & 0.0 & 40.0 & 0.0 \\
$0.5 \mathrm{a}_{0}$ & 10.091 & & $0.55 \mathrm{a}_{0}$ & 8.082 & \\
$0.95 \mathrm{a}_{0}$ & 0.509 & & $0.95 \mathrm{a}_{0}$ & 0.452 & \\
$1.35 \mathrm{a}_{0}$ & 0.086 & & $1.35 \mathrm{a}_{0}$ & 0.132 & \\
$2.05 \mathrm{a}_{0}$ & 0.000 & 0.0 & $2.05 \mathrm{a}_{0}$ & 0.000 & 0.0 \\
& & & & & \\
$\rho$ & $\mathrm{F}(\rho)$ & $\mathrm{F}^{\prime \prime}(\rho)$ & $\rho$ & $\mathrm{F}(f)$ & $\mathrm{F}^{\prime \prime}(\rho)$ \\
\hline 0.0 & 0.0 & 0.0 & 0.0 & 0.0 & 0.0 \\
$0.5 \bar{\rho}$ & -7.123 & & $0.5 \bar{\rho}$ & -7.128 & \\
$1.0 \bar{\rho}$ & -11.374 & & $1.0 \bar{\rho}$ & -10.757 & \\
$2.0 \bar{\rho}$ & -7.521 & & $2.0 \bar{\rho}$ & -6.705 & \\
$2.25 \bar{\rho}$ & 0.0 & 0.0 & $2.25 \bar{\rho}$ & 0.0 & 0.0 \\
\hline \hline
\end{tabular}

Table 2.3: Quantities used for determination of the functions $F(\rho)$ and $Z(r)$ for $Z r$ and their fitted values. Lattice constant $\mathrm{a}_{0}$ and $\mathrm{c}$ in $\AA$; elastic constants $\mathrm{C}_{11}, \mathrm{C}_{33}, \mathrm{C}_{44}, \mathrm{C}_{12}$ and $\mathrm{C}_{13}$ in $10^{12} \mathrm{dyn} / \mathrm{cm}^{2}$; cohesive energy in $\mathrm{eV}$ per atom.

$a$ Ref. [108]

${ }^{b}$ Ref. [60]

\begin{tabular}{cccc}
\hline & EAM1 & EAM2 & Expt. \\
\hline $\mathrm{a}_{0}$ & 3.196 & 3.186 & $3.23^{a}$ \\
$\mathrm{c} / \mathrm{a}$ & 5.019 & 4.986 & $5.15^{a}$ \\
$\mathrm{C}_{11}$ & 1.630 & 1.549 & $1.44^{b}$ \\
$\mathrm{C}_{33}$ & 1.824 & 1.769 & $1.65^{b}$ \\
$\mathrm{C}_{44}$ & 0.362 & 0.359 & $0.321^{b}$ \\
$\mathrm{C}_{12}$ & 0.754 & 0.739 & $0.720^{b}$ \\
$\mathrm{C}_{13}$ & 0.693 & 0.701 & $0.65^{b}$ \\
$\mathrm{E}_{\text {coh. }}$ & 6.250 & 6.510 & $6.25^{a}$ \\
\hline \hline
\end{tabular}




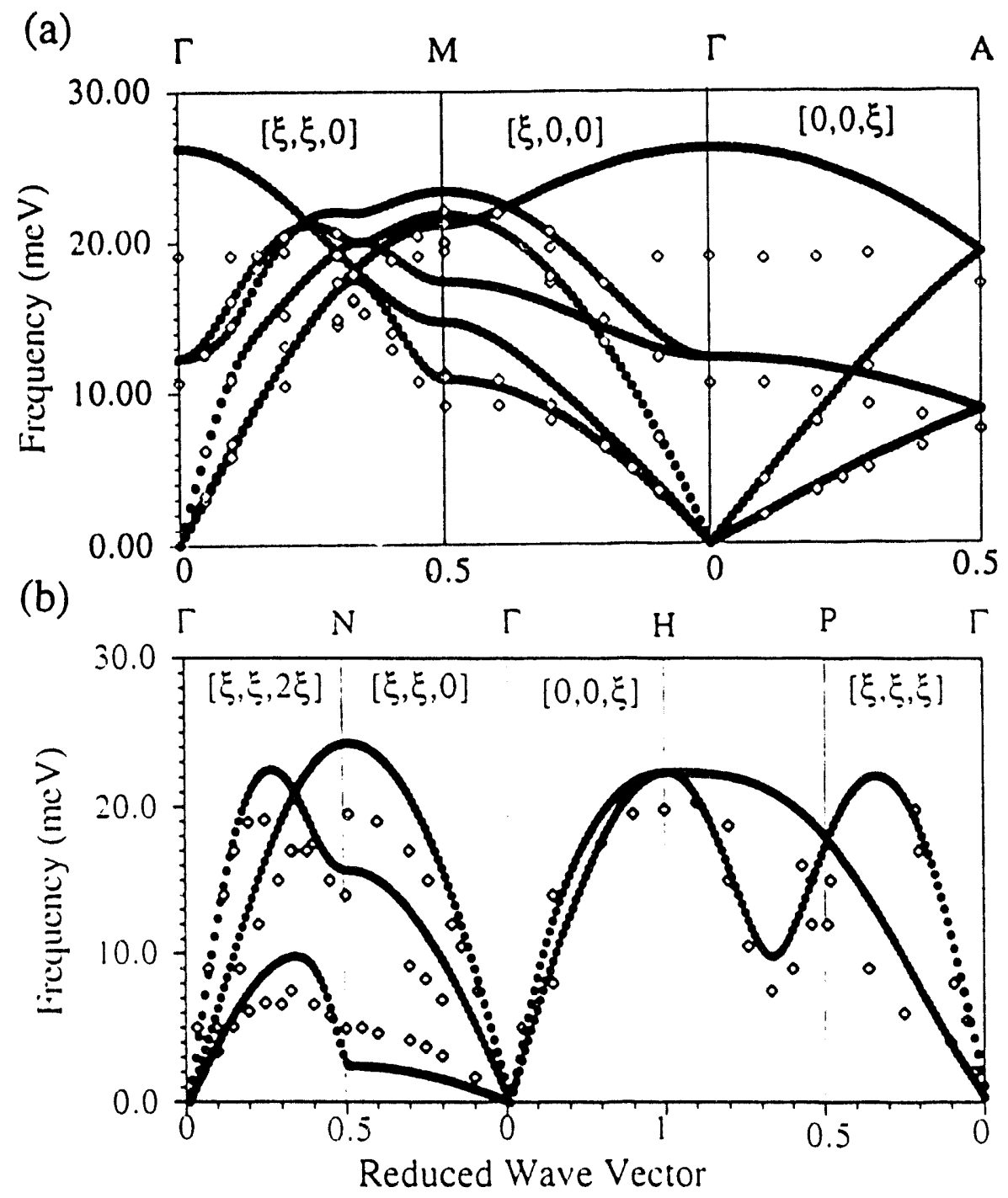

Figure 2.1: Phonon dispersion curves of (a) $h c p$ and (b) bcc $Z$ r for potential EAM1. The solid dots represent the results calculated from lattice dynamics at zero temperature and the empty diamonds are the experimental measurements at $295 \mathrm{~K}$ for $h c p$ [62] and $1188 \mathrm{~K}$ for bcc [21]. 

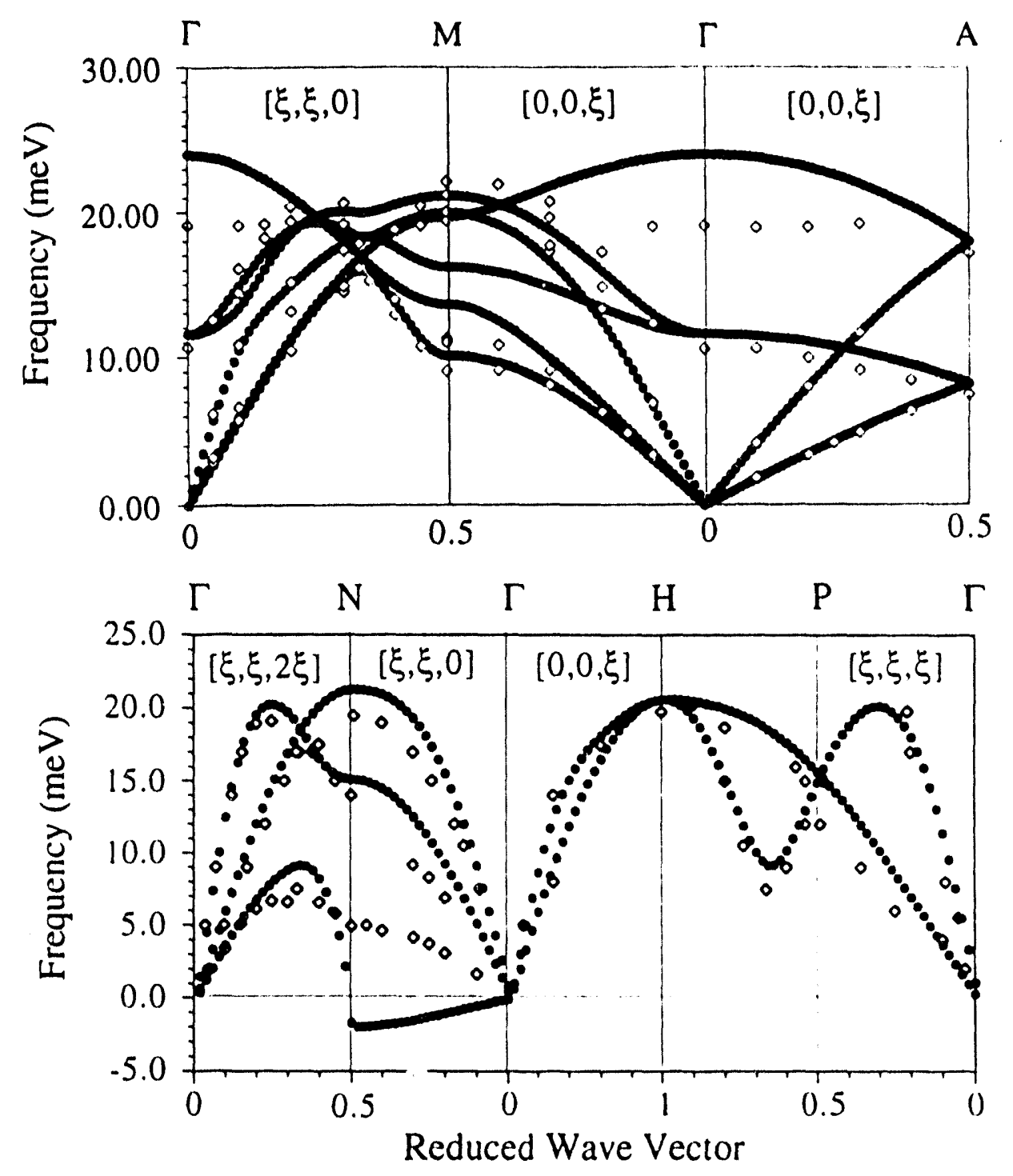

Figure 2.2: Phonon dispersion curves of (a) $h c p$ and (b) bcc $\mathrm{Zr}$ for potential EAM2. The solid dots represent the results calculated from lattice dynamics at zero temperature and the empty diamonds are the experimental measurements at $295 \mathrm{~K}$ for $h c p[62]$ and $1188 \mathrm{~K}$ for bcc [21]. 


\section{CHAPTER 3. MOLECULAR DYNAMICS SIMULATION}

\section{Molecular Dynamics}

In the previous chapter, we have developed an embedded-atom potential for $\mathrm{Zr}$ and showed that it gave satisfactory results for some physical properties. However the above calculations are mostly static and restricied to zero temperature. To compare with experimental results, a dynamical simulation of the finite temperature system is desirable and molecular dynamics (MD) is a very powerful technique to achieve this goal.

In molecular dynamics, the statistical ensemble average of classical interacting many-body system is obtained by sampling a sequence of configurations connected by the coupled Newton's equations of motion

$$
m_{i} \frac{d^{2} \mathbf{r}(t)}{d t^{2}}=-\mathbf{F}_{i}
$$

This set of coupled differential equations is solved via a finite difference approach [63]. The time step is chosen such that it is small enough compared with the cycle of the highest vibrational mode in the system, but as large as possible to simulate a longer time period. The trajectories, $\{\mathbf{r}(\mathrm{t}), \mathbf{p}(\mathrm{t})\}$, provide the essential information in phase space to calculate other physical properties.

We used Gear's 6-th order predictor-corrector algorithm [64] for the numerical 
integration of the equations of motion of the system. Assuming that the classical trajectory is continuous, an estimate of the positions, velocities, etc. at time $t+\delta \mathrm{t}$ is obtained by Taylor expansion about time $\mathrm{t}$

$$
\frac{d^{n}}{d t^{n}} \mathbf{r} P(t+\delta t)=\sum_{k=0}^{5-n} \frac{\delta t^{k}}{k !} \frac{d^{k}}{d t^{k}} \mathbf{r}(t), \quad n=0,1,2, \ldots, 5,
$$

here, the superscript $P$ means that these are the predicted values. The above equation will not generate correct trajectories as time advances, because the equations of motion have not been introduced yet. The correct forces at time $t+\delta t$ are calculated from the new predicted positions $r P$, and hence the temporary acceleration $\frac{d^{2}}{d t^{2}}{ }^{2}{ }^{T}(t+$ $\delta t)$. These are then compared with the predicted accelerations from Equation 3.2 to calculate correction parameters via

$$
\Delta F=\frac{1}{2} \delta t^{2}\left[\frac{d^{2}}{d t^{2}} r^{T}(t+\delta t)-\frac{d^{2}}{d t^{2}} r^{P}(t+\delta t)\right] .
$$

The predicted parameters computed in Equation 3.2 are now corrected with Gear coefficients $G_{i}$ via

$$
\begin{gathered}
\frac{d^{n}}{d t^{n}} r C_{(t+\delta t)=\frac{d^{n}}{d t^{n}} r} P(t+\delta t)+\frac{n !}{\delta t^{n}} G_{n} \Delta F, n=0,1,2, \ldots, 5 \\
G_{0}=\frac{3}{16}, G_{1}=\frac{251}{360}, G_{2}=1, G_{3}=\frac{11}{18}, G_{4}=\frac{1}{6}, G_{5}=\frac{1}{60} .
\end{gathered}
$$

The superscript $\mathrm{C}$ denotes the corrected positions. Although, in principle, this corrector step may be iterated to further refine the trajectory, it is commonly not used since the force calculation is expensive and the single corrector step is usually accurate enough for small time steps. These new corrected values are taken as the current positions, velocities, etc., and all analyses at this time step are performed using these values. This entire procedure is then repeated for all the time steps in the duration of the simulation. 
The information we extract from molecular dynamics simulation can be roughly divided into following groups:

(1) Thermal averages:

$$
<F>=\frac{1}{M} \sum_{i=1}^{N} F\left(t_{i}\right)
$$

such as average total energy:

$$
<E>=\frac{1}{M} \sum_{j=1}^{M}\left[\sum_{i=1}^{N} \frac{p_{i}^{2}\left(t_{j}\right)}{2 m_{i}}+\Phi\left(r\left(t_{j}\right)\right)\right],
$$

here $\mathrm{M}$ is total number of time steps and $\mathrm{N}$ is total number of atoms in the system.

(2) Fluctuations:

$$
(\Delta F)^{2}=<F^{2}>-<F>^{2}
$$

such as heat capacity

$$
C_{v}=\frac{<H^{2}>-<H>^{2}}{k T^{2}}
$$

(3) Time evolution:

$$
F(t)=F(r(t), p(t)) .
$$

(4) Correlation functions:

$$
f(t)=<F(t) F(0)>=\frac{1}{M} \sum_{j=1}^{M} F\left(t+\tau_{j}\right) F\left(\tau_{j}\right),
$$

such as the velocity autocorrelation function

$$
\phi(t)=\frac{1}{M} \sum_{j=1}^{M} v\left(t+\tau_{j}\right) v\left(\tau_{j}\right)
$$




\section{Constant Pressure and Constant Temperature Simulation}

One of the advantages of molecular dynamics is the inclusion of temperature effects. The temperature is generally defined as the average kinetic energy of system per degree of freedom:

$$
\frac{3}{2} k_{B} T=\frac{1}{2 N} \sum_{i}^{N} m\left(\dot{x}_{i}^{2}+\dot{y}_{i}^{2}+\dot{z}_{i}^{2}\right)
$$

Temperature control is usually achieved by simple velocity scaling [65] or by the stochastic random sampling [66] method. In the first method, the velocities of all atoms are scaled by a constant which is the ratio of the desired temperature and average kinetical energy.

$$
v_{i}=C v_{i}, i=1, N
$$

where

$$
C=\frac{\frac{1}{2} \sum_{i=1, N} m_{i} v_{i}^{2}}{3 / 2 N K_{B} T}
$$

This method is in contradiction with the randomness of thermal dynamical system, In the second method, the velocity of each atom is reassigned by a random function to ensure the average kinetic energy to the desired value. However, in the stochastic sampling, there is no histogram in phase space since the system from one step to the next is no longer related. So in both methods, the system is disturbed by an artificial external force and the time correlation functions are not accurate. In our simulations, the system is warmed up to the desired temperatures by using one of the above methods and the system is allowed to equilibrate for sufficiently long time. The correlation functions are then calculated with a constant energy (microcanonical ensemble) simulation with no temperature control. 
In the early 1980 's, there were several proposals $\{67,68,69\}$ for constant temperature simulation and the one mostly used was proposed by Nosé in $1983[69]$. In Nosé's scheme, the extended system has an extra degree of freedom $s$ and the new Hamiltonian is postulated as

$$
H=\sum_{i} \frac{P_{i}^{2}}{2 m_{i} S^{2}}+\phi(q)+\frac{p_{s}^{2}}{2 Q}+g k_{B} T \ln s,
$$

$p_{s}$ is the conjugate momentum of $s ; \mathrm{Q}$ is a parameter of dimension energy $(\text { time })^{2}$ and behaves as a mass for the motion of $s ; T$ is the externally set temperature; the parameter $g$ is essentially equal to the number of degrees of freedorn of the physical system. The extra degree of freedom $s$ serves as the link between the system and the outside heat bath in the way that the time step is scaled by $s$ every step. In the long time limit, it was proved that the simulation by Nosés scheme produces the canonical ensemble 70$]$. We have implemented Nosé scheme for simulating the melting of the $h c p \mathrm{Zr}$. The result is very close (within $100 \mathrm{~K}$ ) to the one we got from the simple velocity scaling method. But the Nosé scheme gives the rigorous canonical distribution both in momentum and in coordinate space; it can be more significant in other calculations of dynamical properties.

Another important aspect of MD is the unit cell. In the early stages of molecular dynamics, the unit cell was fixed and thus MD simulations cannot study physical problems involving changes in the volume and shape of the system. In 1980, Anderson 66 proposed a scheme for carrying out MD simulation allowing the volume of the unit cell to vary in time. Later, Parrinello and Rahman [71] generalized Anderson's scheme to incorporate the simultaneous change of size and shape of the unit cell. The unit cell can be completely described by three vectors $\mathbf{a}, \mathbf{b}$, and $\mathbf{c}$ that span the edge 
of the MD cell. The length and orientation of $\mathbf{a}, \mathbf{b}$ and $\mathbf{c}$ can be described by $\mathbf{a} 3 \times 3$ matrix $h$ and the volume of the unit cell is given by

$$
\Omega=\|\mathbf{h}\|=\mathbf{a} \cdot(\mathbf{b} \times \mathbf{c})
$$

The position $\mathbf{r}_{i}$ of a particle $i$ can be written in terms of $\mathbf{h}$ and a column vector $\mathbf{s}_{\mathbf{i}}$, with components $\xi_{i}, \eta_{i}$, and $\zeta_{i}$, and

$$
\mathbf{r}_{i}=\mathbf{h} \mathbf{s}_{i}=\xi_{i} \mathbf{a}+\eta_{i} \mathbf{b}+\zeta_{i} \mathbf{c}
$$

Then the distance between particle $i$ and $j$ is given by

$$
r_{i j}^{2}=\left(\mathbf{s}_{i}-\mathbf{s}_{j}\right)^{T} \mathbf{G}\left(\mathbf{s}_{i}-\mathbf{s}_{j}\right)
$$

where the metric tensor $\mathbf{G}$ is

$$
\mathbf{G}=\mathbf{h}^{\mathbf{T}} \mathbf{h}
$$

Here the ${ }^{T}$ denotes a transpose of a vector or a tensor in the usual way. The usual set of $3 \mathrm{~N}$ dynamical variables, that describe the positions of $\mathrm{N}$ particles, was augmented by the nine components of $h$. The time evolution of the $3 N+9$ variables was then obtained from the Lagrangian

$$
\mathcal{L}=\frac{1}{2} \sum_{i=1}^{N} m_{i} \dot{\mathbf{s}}_{\mathbf{i}}^{\mathrm{T}} \mathbf{G} \dot{\mathbf{s}}_{\mathbf{i}}-\sum_{i=1}^{N} \sum_{j>i}^{N} \phi\left(r_{i j}\right)+\frac{1}{2} W \operatorname{Tr}\left(\dot{\mathbf{h}}^{\mathrm{T}} \dot{\mathbf{h}}\right)-p \Omega
$$

where $p$ is the hydrostatic pressure, $W$ is the "wall mass" of the unit cell and the equations of motion are

$$
\begin{gathered}
\ddot{s}_{i}=-\sum_{i \neq j} m_{i}^{-1}\left(\phi^{\prime} / r_{i j}\right)\left(\mathbf{s}_{i}-\mathbf{s}_{j}\right)-\mathbf{G}^{-1} \dot{\mathbf{G}} \dot{s}_{i} \\
W \ddot{\mathbf{h}}=(\pi-p) \sigma
\end{gathered}
$$


where tensor $\pi$ and $\sigma$ are defined as

$$
\begin{gathered}
\Omega \pi=\sum_{i} m_{i} \mathbf{v}_{i} \mathbf{v i}-\sum_{i} \sum_{j>i}\left(\phi^{\prime} / r_{i j}\right) \mathbf{r}_{i j} \mathbf{r}_{i j} \\
\sigma=\Omega \mathbf{h}^{\mathbf{T}-1}
\end{gathered}
$$

The above Lagrangian generates a constant pressure and constant enthalpy $(p, \mathrm{H}, \mathrm{N})$ ensemble and can be easily extended to $(t, \mathrm{H}, \mathrm{N})$ ensemble, where $t$ stands for the external stress [71].

With the above extension, we can simulate some very important physical processes, such as thermal expansion, structural phase transitions and mechanical properties of materials under external pressure and stress.

\section{Thermal Expansion and Melting}

Using the Parrinello-Rahman scheme, we studied the thermal expansion of $h c p$ Zr. For the $h c p$ lattice, the unit cell was chosen to be rectangular with $\mathbf{a}, \mathbf{b}$ and $\mathbf{c}$ along [001], [100] and [010] directions respectively. The unit cell contained 800 atoms and the external pressure was set to zero. For a given temperature, we ran 4000 steps to let the system reach thermal equilibrium and another 4000 steps to get the averaged lattice constants. In all the simulations, the fictitious wall mass parameter for the cell was set equal to 10 times of the mass of a $\mathrm{Zr}$ atom and the time step is $1.95 \times 10^{-15}$ second.

The lattice constants as function of temperature are plotted in Fig. 3.1. Overall, our results overestimate the thermal expansion. This may due to the fact that the potential is only fitted to the properties at equilibrium volume. 
Another physical phenomenon that can be investigated is the melting of the crystal. We monitor the potential energy of the system as a function of temperature, a sudden jump in this quantity occurs when the system melts. The results are plotted in Fig. 3.2 and the melting temperature is estimated be about $2500 \mathrm{~K}$. This value is far higher than the experimental result of $1135 \mathrm{~K}$ and this is quite common for MD simulations [73]. It is mainly due to the lack of a surface in simulations (our three dimentional periodic boundary condition generates an infinite single crystal). However, in experimental conditions, melting starts from the surface, defects or grain boundaries. In MD studies, superheating above the thermodynamic melting point $\mathrm{T}_{m}$ can be a significant fraction of $\mathrm{T}_{m}[74]$. The overestimation also comes from the limitation of simulation time compared to the real melting process. This point will be further discussed in Chapter 8. 

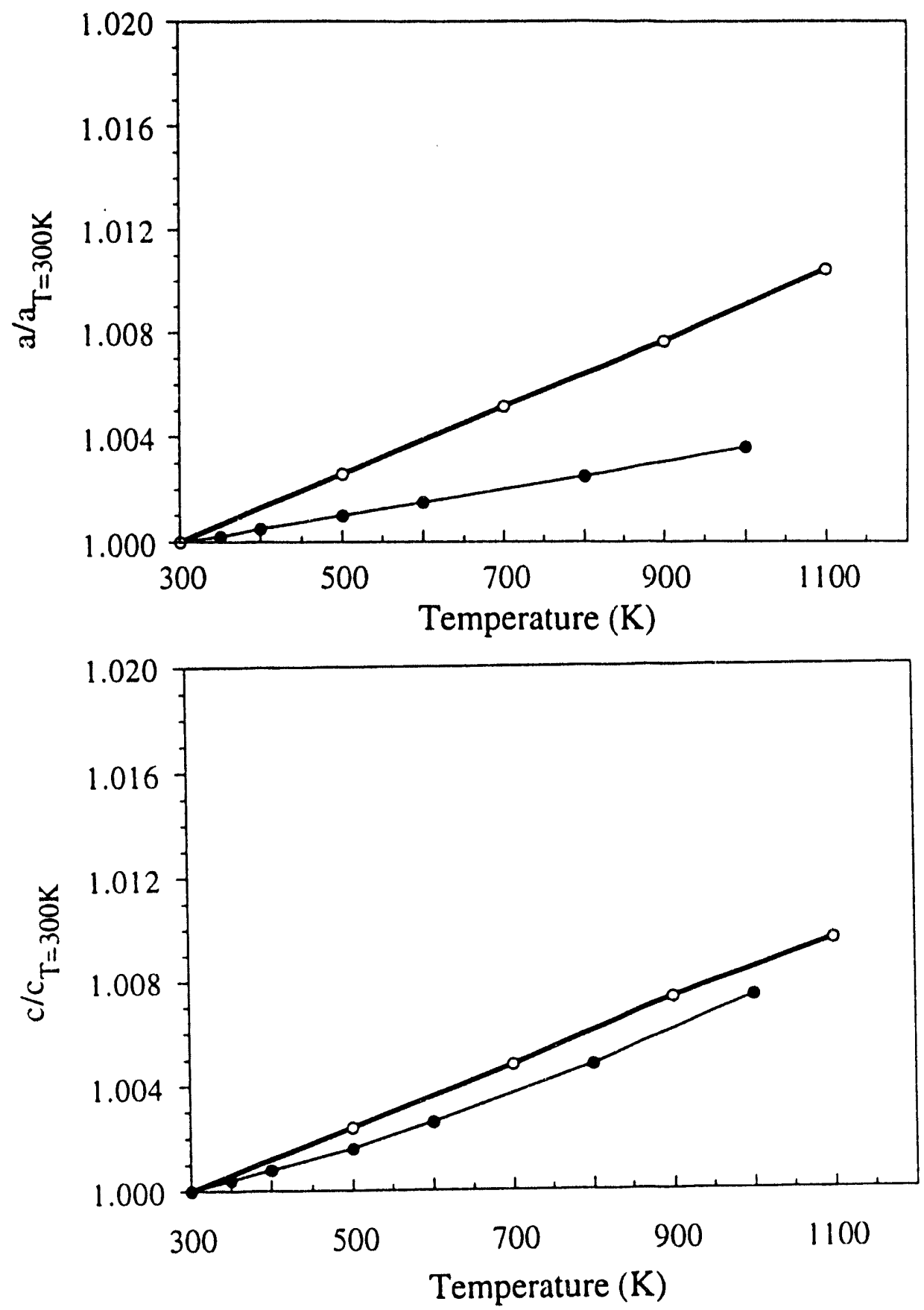

Figure 3.1: The lattice constants of $h c p \mathrm{Zr}$ as function of temperature. The open circles are the results of our simulation and the corresponding results of experimental measurements [72] are represented by solid circles. 


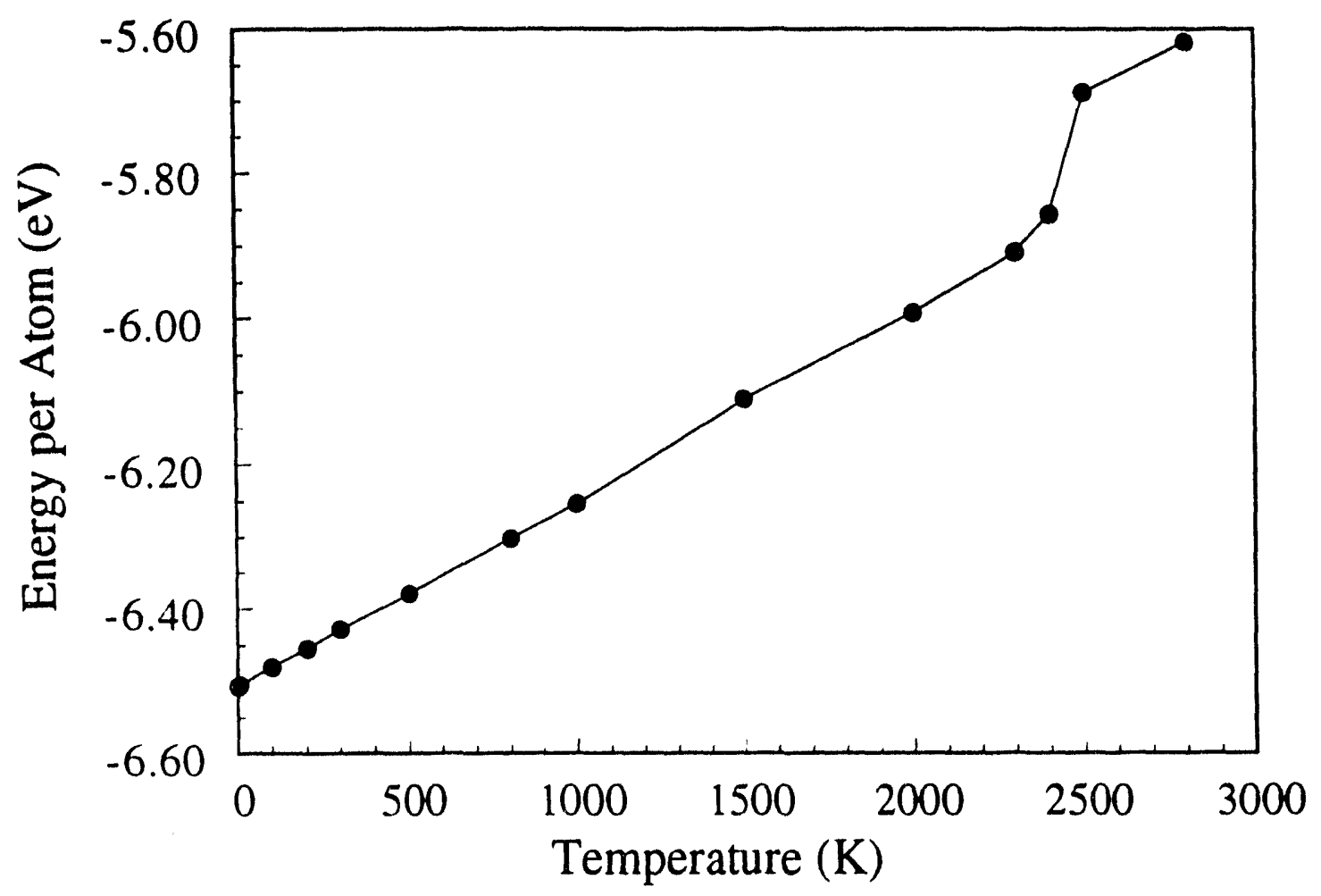

Figure 3.2: Total energy of $h c p \mathrm{Z}_{\mathrm{r}}$ as function of temperature. 


\section{CHAPTER 4. ANOMALOUS PHONON BEHAVIOR AND PHASE FLUCTUATION OF BCC ZR}

\section{Introduction}

Martensitic phase transitions involve a collective displacement of atoms through distances of the order of the unit cell length or smaller. Thus the transition occurs in a diffusionless way. Sometimes, the displacements corresponding to certain phonon modes are included as a part of the collective motion. Thus, the relations between phonon behavior and the phase transition in martensitic systems have attracted a lot of attention from both experimentalists and theorists $[1,2,3,4,5,6,7,45,12$, $13,14,15,16,17]$. The high temperature $b c c$ phase $\mathrm{Zr}$ exhibits intrinsic instabilities towards the low temperature $h c p$ phase and high pressure $\omega$ phase. The geometric relations of these structures are illustrated in Fig. 4.1. The displacement associated with the $\mathrm{T}_{1}$ mode of the $N$ point phonon in the $b c c$ phase is related to the shuffle of every second [110] plane. According to Burgers' mechanism [75], the transition from $b c c$ to $h c p$ is also a shuffle of every second [110] plane with the second plane atom (marked as cross in Fig. 4.1(b)) moving into a position directly above the center of a triangle formed by three first plane atoms. On the other hand, look at the $b c c$ crystal in the [111] direction. The lattice is repeated every three planes as marked in Fig. 4.1(a). If the plane 1's remain stationary, while plane 2's and 3's collapse together 
as depicted in Fig. 4.1(a), the structure is known as the $\omega$ phase. Thus, the phase transition between $b c c$ and $\omega$ can be associated with displacements corresponding to the longitudinal phonon at $\frac{2}{3}(1,1,1)(\omega$ point $)$.

Inelastic neutron scattering measurements on $b c c \mathrm{Zr}$ and $\mathrm{Zr}$ alloys indicated that the longitudinal $[\xi \xi \xi]$ phonon at the $\omega$ point as well as the $\mathrm{T}_{1}$ mode at the $N$ point have very low frequencies and strong quasi-elastic intensities [19, 20, 21]. Another most intriguing feature is that the measured phonon spectra and the quasi-elastic scattering exhibit a strong asymmetry around $(2,2,2)$ bcc Bragg peak along the [11 $\overline{2}]$ direction $[4,5,11]$. The quasi-elastic intensity at $A=\left(\frac{7}{3}, \frac{7}{3}, \frac{4}{3}\right)$ is about 30 times larger than that at $B=\left(\frac{5}{3}, \frac{5}{3}, \frac{8}{3}\right)$. This violates the $b c c$ symmetry which would hold for a normal crystal. This anomalous phonon behavior appears to be generic for a wide range of system in which the bcc phase transforms to another structure at low temperatures $[19,20,21,22,23]$.

In all the previous experiments reported $[4,11,19,20,21]$, the measurements were performed at large wavevectors outside the first and second Brillouin zones to improve the signal over the noise background. For the $\omega$ point phonon, the measurement was actually performed at $\mathbf{Q}=\frac{4}{3}(1,1,1)$ and the $b c c$ periodicity is used to fold the wavevector back to the "equivalent" $\boldsymbol{\omega}$ point $\left(\mathbf{Q}=\frac{4}{3}(1,1,1)=(2,2,2)-\mathbf{Q}=\frac{2}{3}(1,1,1)\right)$. The basic assumption here is that the measured cross-sections should be the same in different Brillouin zones. However, it may not be true when the system is highly anharmonic.

It was generally accepted that the $b c c$ to $\omega$ phase transition is the major factor for the above anomalous phonon behavior. The strongest evidence for the importance of the $\beta$ to $\omega$ transition is the experimental observation of quasi-elastic peaks at 

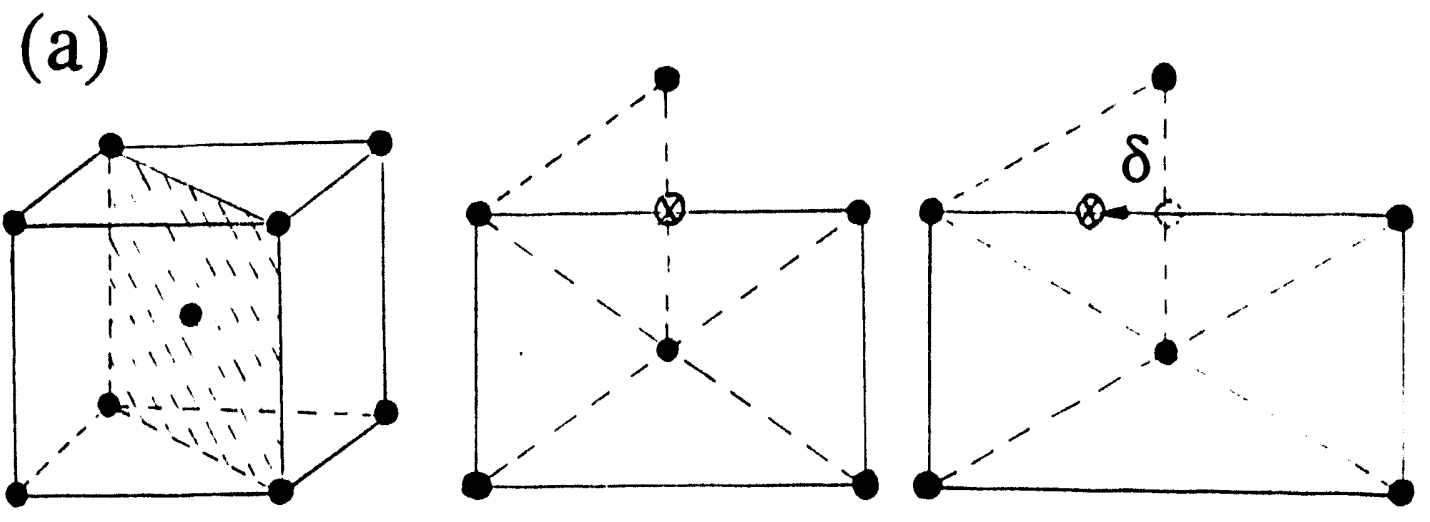

(b)

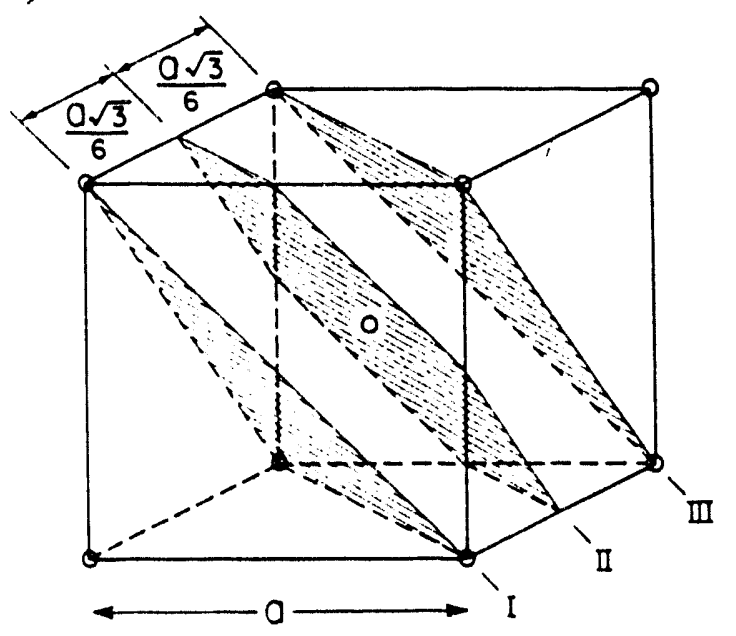

123123123

|L1!!|!|| b bec Iiiliili ili PHONON | | | | | | WPAASE

Figure 4.1: (a) The geometric relation between bcc and $h c p$ phase. (b) Geometric relation between $b c c$ and $\omega$ phase. 
positions corresponding to the Bragg peaks of the $\omega$ phase. However, in the recent work of Heiming et al, they found that the quasi-elastic peak is not at exactly $\mathbf{Q}=\frac{4}{3}(1,1,1)$ but at $\mathbf{Q}=1.28(1,1,1)$. And with an appropriate $\mathrm{c} / \mathrm{a}$ ratio, this $\mathbf{Q}$ point is also a Bragg peak from the $h c p$ phase. So Heiming et al. $\{21\}$ argued that this quasi-elastic scattering is not from the $\omega$ phase but from the $h c p$ phase. Since there are no $h c p$ Bragg peaks around real $\omega$ point, data at the real $\omega$ point becomes crucial for determining the origin of these quasi-elastic scattering. An observation of quasielastic scattering at the real $\omega$ point will unambiguously confirm the presence of fluctuations from the $\beta$ phase to the $\omega$ phase while a negative result will exclude this kind of fluctuation.

Finally, both $b c c$ to $\omega$ and $b c c$ to $h c p$ phase transitions can not account the asymmetry observed in neutron scattering. Although there are a lot of efforts to explain this interesting anomaly, a clear physical picture is still lacking.

\section{Simulation of Phonon Spectrum}

The phonon spectra are calculated directly from the dynamical structure factor, defined as $\{76\}$

$$
S(\mathbf{Q}, \omega)=\frac{1}{N} \sum_{\mathbf{R} \mathbf{R}^{\prime}} e^{-i \mathbf{Q} \cdot\left(\mathbf{R}-\mathbf{R}^{\prime}\right)} \int \frac{d t}{2 \pi} e^{i \omega t}\left\langle\exp \left[i \mathbf{Q} \cdot \mathbf{u}\left(\mathbf{R}^{\prime}, 0\right)\right] \exp [-i \mathbf{Q} \cdot \mathbf{u}(\mathbf{R}, t)]>\right.
$$

which is proportional to the measured inelastic neutron scattering cross-section. In Equation $4.1, \mathbf{u}(\mathbf{R}, t)$ is the displacement of the atom from the lattice position $\mathbf{R}$ at time $t$. For small displacements, we can expand the above expression in powers of 
$\mathbf{Q} \cdot \mathbf{u}$

$$
\exp <[\mathbf{q} \cdot \mathbf{u}(0)][\mathbf{q} \cdot \mathbf{u}(\mathbf{R}, t)]>=\sum_{m=0}^{\infty} \frac{1}{m !}(<[\mathbf{q} \cdot \mathbf{u}(0)][\mathbf{q} \cdot \mathbf{u}(\mathbf{R}, t)]>)^{m}
$$

For $m=0$, it is simply the Bragg reflection and the one-phonon contribution is from the term $m=1$

$$
<[\mathbf{q} \cdot \mathbf{u}(0)][\mathbf{q} \cdot \mathbf{u}(\mathbf{R}, t)]>
$$

This is equivalent to the velocity-velocity correlation when the system is harmonic and the phonon peak is well-defined. The one-phonon contribution to the dynamical structure factor is the same at equivalent points in different Brillouin zones in reciprocal space except for a simple scaling factor. Due to the noise at high temperature, it is usual to measure the scattering at $q+G$, where $G$ is a bcc reciprocal lattice vector, and fold it back to $q$. But when $\mathbf{u}$ becomes large at high temperature, the above expansion 4.2 is not guaranteed to converge after the one-phonon term. The spectrum at bigger $\mathbf{Q}(\mathbf{Q}=\mathbf{q}+\mathbf{G})$ obviously introduces more multi-phonon contribution than that at small $\mathbf{q}$. Then the spectra measured at different zones will not only have different intensities but also different shapes.

The scattering cross-sections measured in inelastic neutron experiments correspond to the absolute square of the dynamical structure factor given in Equation 4.1. Thus our calculations include anharmonic effects exactly without the approximations involved in traditional anharmonic calculations using self-consistent phonon theory or displacement-displacement (velocity-velocity) correlations $[77,78]$. When the one-phonon contribution dominates, all of the above techniques yield the same answer. This is illustrated by selected phonon spectra at different temperatures for the $h c p$ lattice (see Fig 4.2). For small $\mathbf{q}$, the dynamical structure factor is almost 
the same as the velocity-velocity correlation function at low temperature. The shape of the spectra at larger $\mathbf{Q}, \mathbf{Q}=\mathbf{G}+\mathbf{q}$ ( $\mathbf{G}$ is the reciprocal vector), is also similar to that of small $\mathbf{q}$.

As the temperature increases, the phonon peaks become broad. Also the spectra of the dynamical structure factor deviate more from the spectra obtained from velocity-velocity correlation function. This is an indication that the anharmonic effects become bigger as the temperature increases even though the effect is still rel atively small. Another interesting phenomenon is that most of the phonon modes become soft as the temperature increase. This is mainly due to the thermal expansion of the lattice, which weakens the restoring forces of vibrations. From Fig. 4.2, the phonon at $\frac{2 \pi}{c}\left(0,0, \frac{1}{6}\right)$ shifts about $0.05 \mathrm{meV}$ which is relatively small compared to the experimental value of $0.37 \mathrm{meV}[19]$.

\section{Anomalous Phonon Behavior and Phase Fluctuation at high temperature $b c c$ phase}

While the $h c p$ crystal behaves quite regularly, bcc $\mathrm{Zr}$ exhibits quite anomalous phonon behavior. By using the Parrinello-Rahman constant pressure scheme, we have studied the stability of the $b c c$ phase. For potential EAM1, the bcc lattice is metastable at very low temperature but transforms to the $h c p$ when the temperature is higher than $400 \mathrm{~K}$. For potential EAM2, the bcc lattice is not stable at any temperature since one phonon branch is totally soft (see Fig. 3.2). In both cases, the transformation from $b c c$ to $h c p$ becomes slower as the temperature increases. This is due to the lattice-vibration entropy which tends to stabilize the bcc structure. Since the electronic entropy, which also plays an important role in stabilizing the bcc 


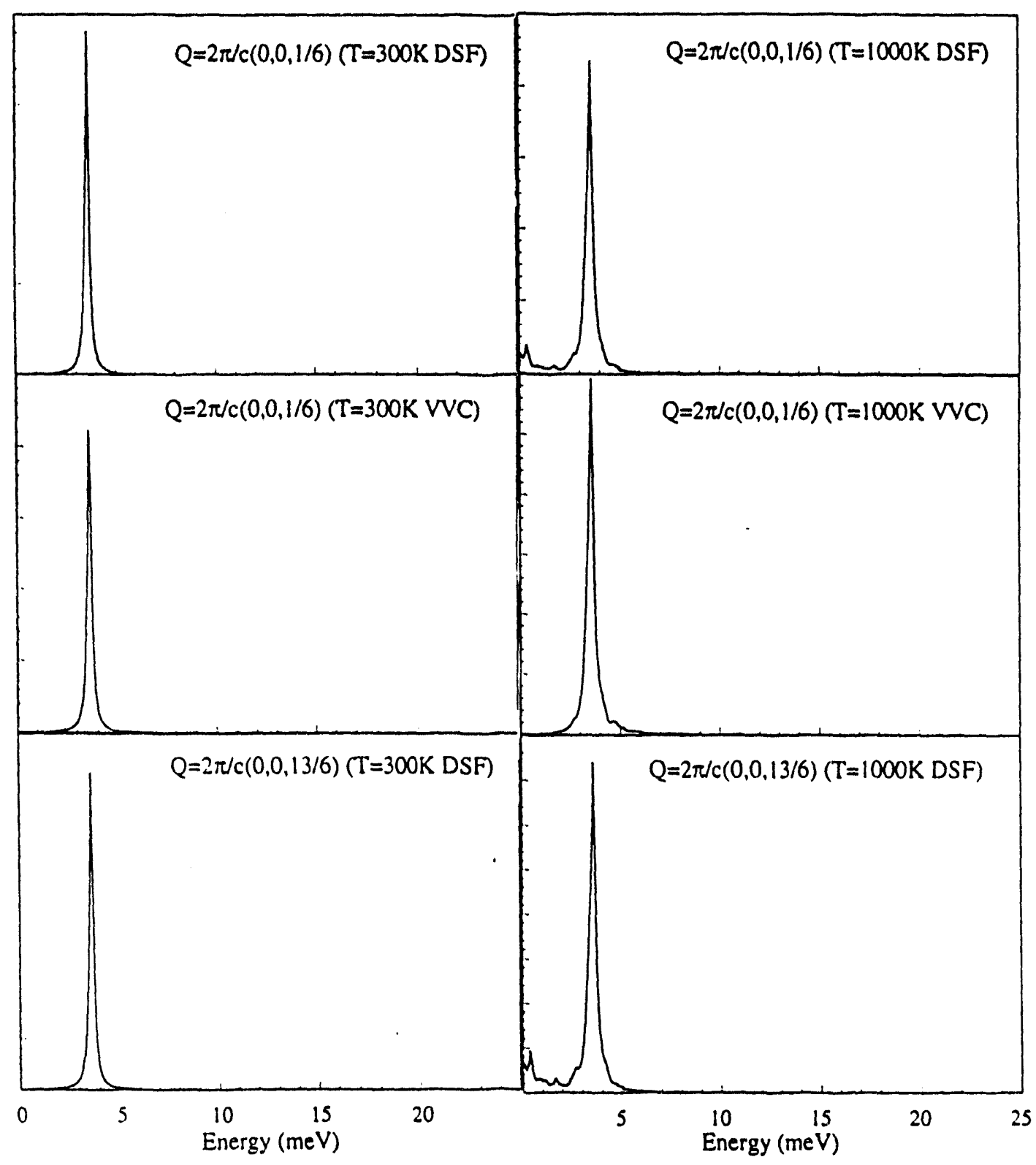

Figure 4.2: The phonon spectra of $h c p \mathrm{Z}_{\mathrm{r}}$ calculated from dynamical structure factor (DSF) and velocity-velocity correlation (VVC) at $300 \mathrm{~K}$ and $1000 \mathrm{~K}$. 
structure [79], is not included in our potential models, we cannot stabilize the bcc structure before the systems melt. However, for both potentials, the bcc lattice can be stabilized by constant volume simulation and this enables us to study the behavior of the system at high temperatures.

A very useful piece of structural information which can be extracted from molecular dynamics is the radial distribution function which is defined as

$$
N(r)=4 \pi \bar{\rho} \int G(r) r^{2} d r .
$$

Here $N(r)$ is the number of neighbouring atoms within radius $r$ and $\bar{\rho}$ is the average density $\mathrm{N} / \mathrm{V}$. In molecular dynamics simulation, $\mathrm{G}(\mathrm{r})$ is averaged over all the atoms in the unit cell and over a long time period. Through a Fourier transformation, the $G(r)$ can be converted to the static structure factor $S(Q)$ which can be measured by scattering experiments. For a temperature of $1500 \mathrm{~K}$, the $G(r)$ function is very broad and it is difficult to identify the structure of the system (see Fig. 4.3). Instead, we can look at the $\bar{G}$ (raverage) function that is defined as the radial distribution of atomic positions averaged over a long time period. In our simulation of 8000 steps, the new function $\bar{G}$ (raverage) has very sharp peaks exactly corresponding to the bcc lattice. This means that the system is fluctuating about the $b c c$ lattice even though the fluctuations are very strong. All these functions are plotted in Fig. 4.3 along with the $G(r)$ of ideal $b c c$ and $h c p$ lattices for purposes of comparison.

In Fig. 4.4, the dynamical structure factors at several special Q-points obtained from our calculations are presented. For the $\omega$ point phonon, our simulated $S(\mathbf{Q}, \omega)$ at $\mathbf{Q}=\frac{4}{3}(1,1,1)$ indicates a strongly damped phonon with frequency below $5 \mathrm{meV}$, while the spectrum at $\mathbf{Q}=\frac{2}{3}(1,1,1)$ shows a broad, but still well defined, peak at 11 meV. Thus, we see that it might be misleading to interpret the measured neutron 


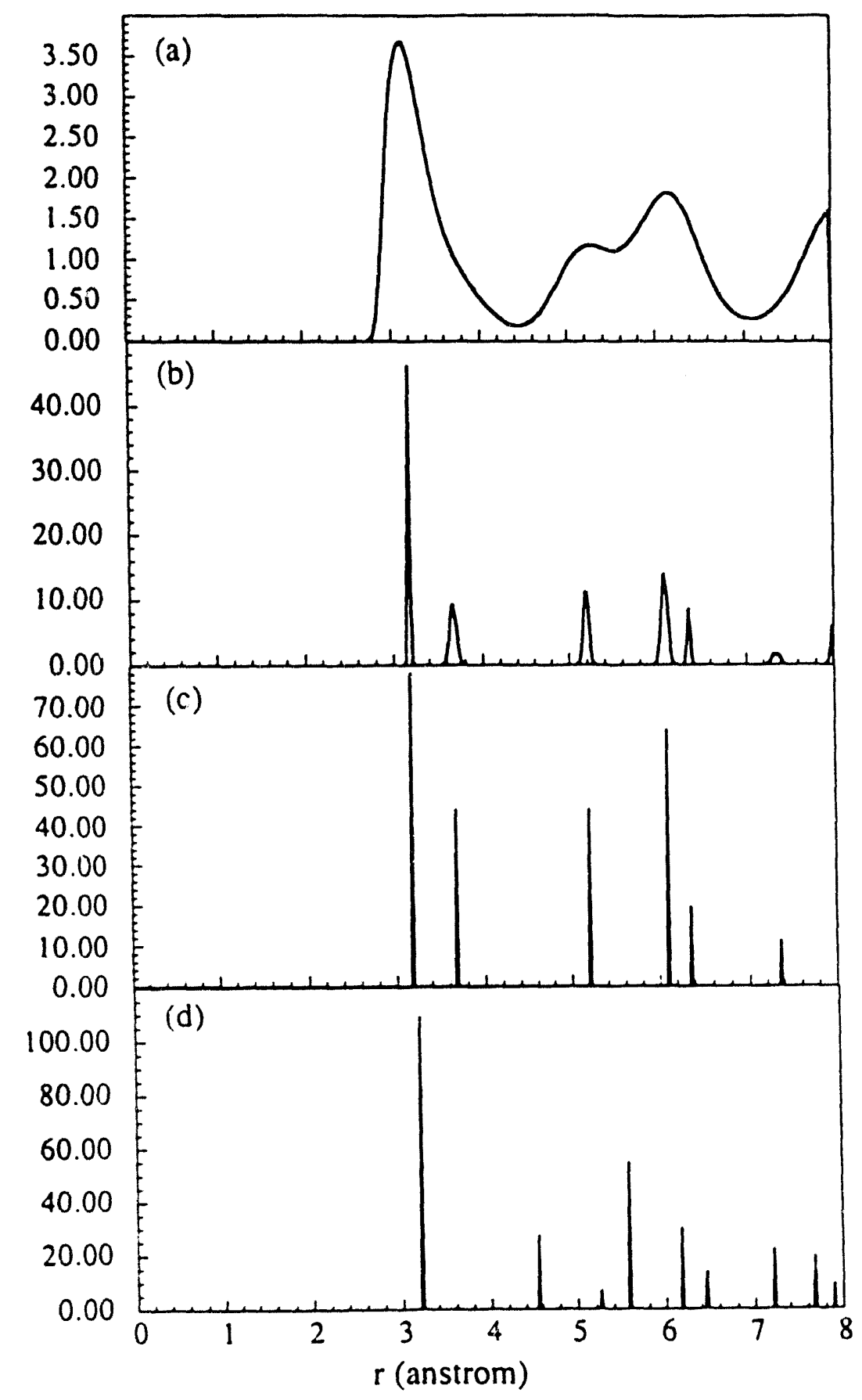

Figure 4.3: (a)G(r) function of bcc $\mathrm{Z}_{\mathrm{r}}$ and (b) $\bar{G}$ (raverage) function of $h c p \mathrm{Z}_{\mathrm{r}}$ at high temperature. The $\mathrm{G}(\mathrm{r})$ functions of $(\mathrm{c})$ ideal $h c p$ and (d) ideal bcc $\mathrm{Z}$. 
scattering cross-section at high wavevectors solely in terms of one-phonon scattering. The lack of quasi-elastic intensity for the dynamical structure factor at $\mathbf{Q}=\frac{2}{3}(1,1,1)$ implies that $\omega$ phase fluctuations are not very strong in our system.

We show in Fig. 4.5(b) a contour plot of the variation of the integrated scattering intensity in the $[1 \overline{1} 0]$ plane in reciprocal space. The intensity is defined as

$$
I(\mathbf{q})=\int_{\omega=0}^{\omega=\omega_{0}} S(\mathbf{q}, \omega) d \omega
$$

where the $\omega_{0}$ is chosen to be $30 \mathrm{meV}$ where $S(\mathbf{q}, \omega)$ almost drops to zero. The wavevector dependence of the quasi-elastic scattering agrees very well with neutron scattering measurements for the $Z r_{0.8} N b_{0.2}$ alloy (Fig. 4.5(a)), in particular, we also observe strong asymmetry about the $(2,2,2)$ bcc Bragg peak along the $\{11 \overline{2} \mid$ directions (between $\mathbf{A}=\left(\frac{7}{3}, \frac{7}{3}, \frac{4}{3}\right)$ and $\mathbf{B}=\left(\frac{5}{3}, \frac{5}{3}, \frac{8}{3}\right)$ ).

While we perform the simulation for a pure $Z_{r}$ crystal at high temperatures, the experiments [4] were performed for quenched $\mathrm{ZrNb}$ alloys. The similarity between the two results supports a picture in which the rapid quenching freezes in the dynamic phase fluctuations occurring at high temperatures. In that case, we expect a strong modification of the frequency dependence, but not much change in the wavevector dependence, between the observed $S(Q, \omega)$ and our calculated results. Recent inelastic neutron scattering experiments on pure bcc La and $\mathrm{Z}_{\mathrm{r}}$ crystals at high temperatures also provide confirmation of our results $\{22,81\}$.

To investigate the nature of the phase fluctuations in the system, we defined an order parameter to measure the instantaneous bcc crystalline order

$$
S(t)=\sum_{j} \sum_{i} e^{i \mathbf{G}_{j} \cdot \mathbf{r}_{i}(t)}
$$




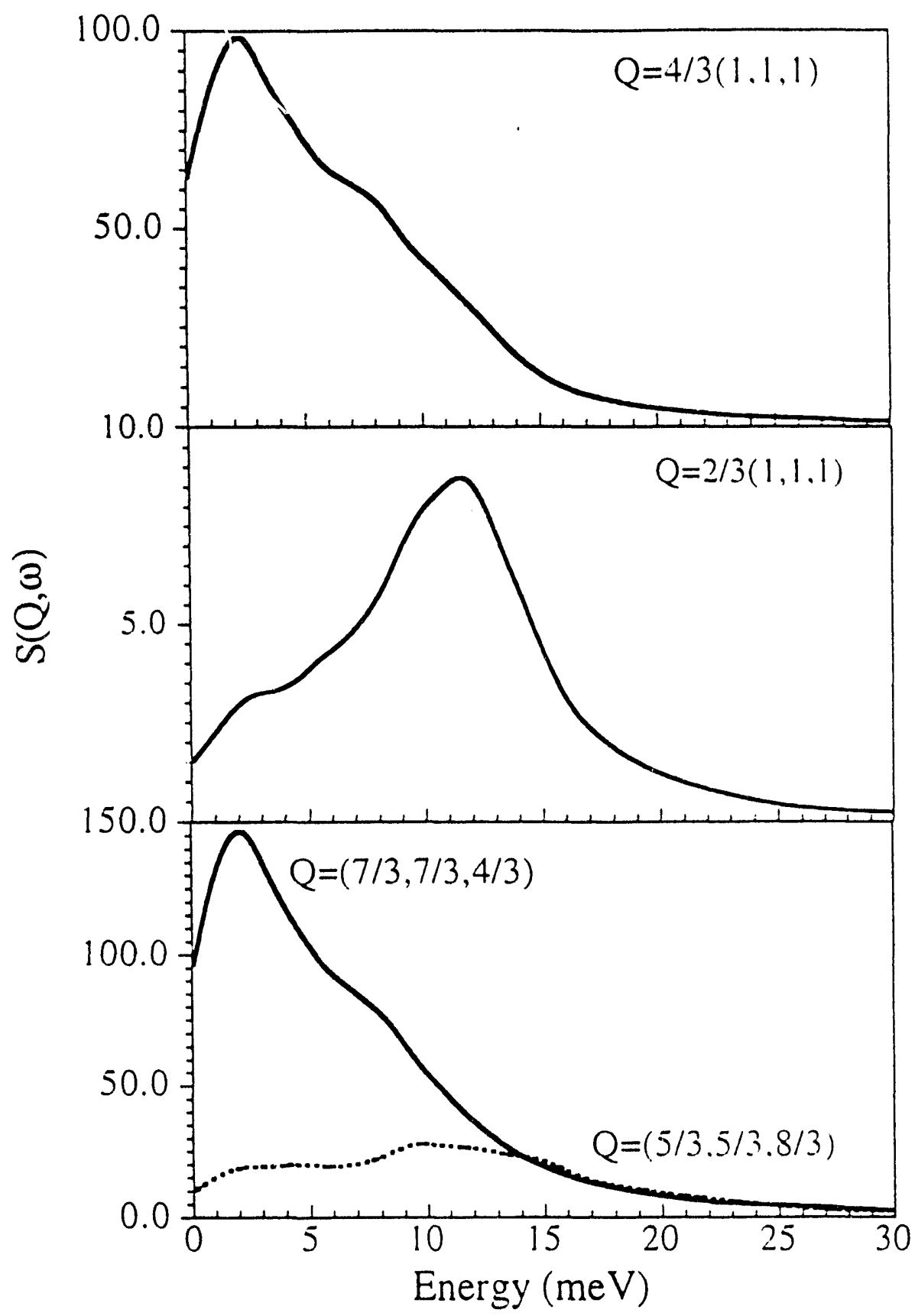

Figure 4.4: Dynamic structure factors at selected $Q$ points. All the spectra are broadened by a Lorentzian function with a width of $1.5 \mathrm{meV}$. 


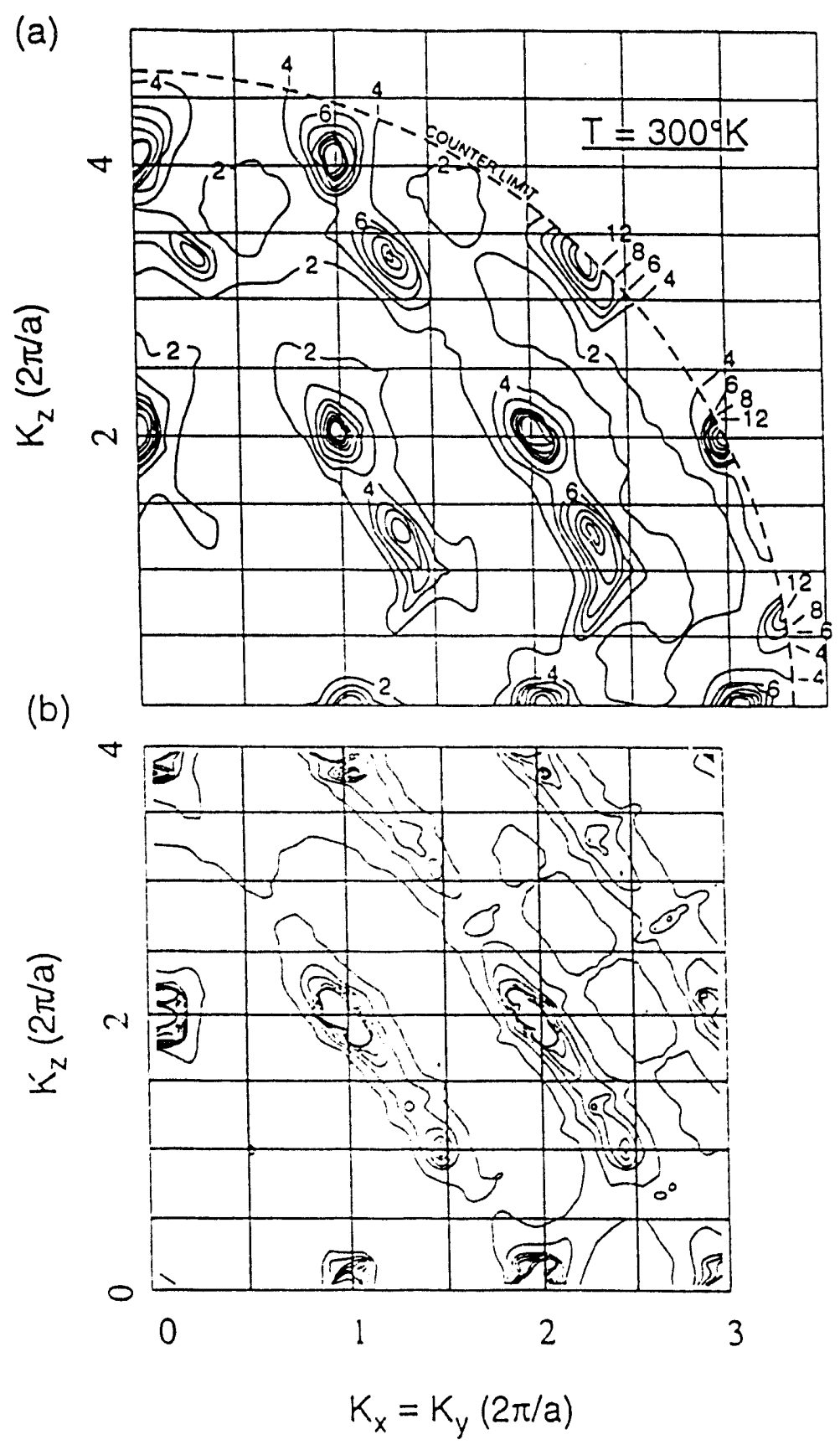

Figure 4.5: Contour plots of the integrated scattering intensity of (a) $\mathrm{Zr}$ crystal simulated by molecular dynamics and (b) $\mathrm{Zr}_{0.8} \mathrm{Nb}_{0.2}$ system measured by inelastic neutron scattering [4]. 
where the summation is over all bcc reciprocal lattice vectors $\mathbf{G}_{j}$ and over all atoms in the system. If all the atoms are on the ideal bcc lattice, $S(t)$ reaches the maximum value $\mathrm{N} \times \mathrm{M}$ where $\mathrm{N}$ is the total number of atoms in the unit cell and $\mathrm{M}$ is the number of reciprocal lattice vectors. As the system drifts away from the ideal lattice, the exponential term is always less than 1 and $S(t)$ decreases. Noting that the radial distribution function of the $h c p$ structure has a peak at about $4.5 \AA$, while that of the $b c c$ structure has none between $4.0 \AA$ and $5.5 \AA$ (Fig. 4.3 ), we also calculate the number of pairs of atoms with separation between $4.2-4.7 \AA A N_{h c p}$, as a measure of the amount of hcp-like fluctuations in the system. The time evolutions of these two functions are plotted in Fig. 4.6. As we can see, there is a strong correlation between fluctuations away from the bcc structure (dips in $S(\mathrm{t})$ ) and the growth of $h c p$-like clusters (peaks in $\left.N_{h c p}(\mathrm{t})\right)$. Up to now, all the experimental information is obtained from scattering which only provides $\mathrm{k}$-space pictures. One of the advantages of molecular dynamics is that we can obtain the real space atomic configurations. A snapshot picture of the atomic configuration at instant when the $N_{h c p}(\mathrm{t})$ has a peak is plotted in Fig. 4.7. By analysing snapshot pictures carefully, we can identify local clusters of atoms arranged in $h c p$-like configurations. These $h c p$ "embryos" disappear as $S(\mathrm{t})$ increases and come back at another $\operatorname{dip}$ of $S(\mathrm{t})$. The locations, as well as the directional variation of these embryos change with time and this is consistent with the good $b c c$ lattice we obtained from the long-time average. If the system fluctuates between $h c p$ and $b c c$ via one directional variant, the average atomic positions must be somewhere between the $b c c$ and $h c p$ lattices instead of the ideal bcc lattice.

The dynamical fluctuations of the system between the bcc and the hcp phase is the microscopic origin of the asymmetry of the quasi-elastic scattering in reciprocal 


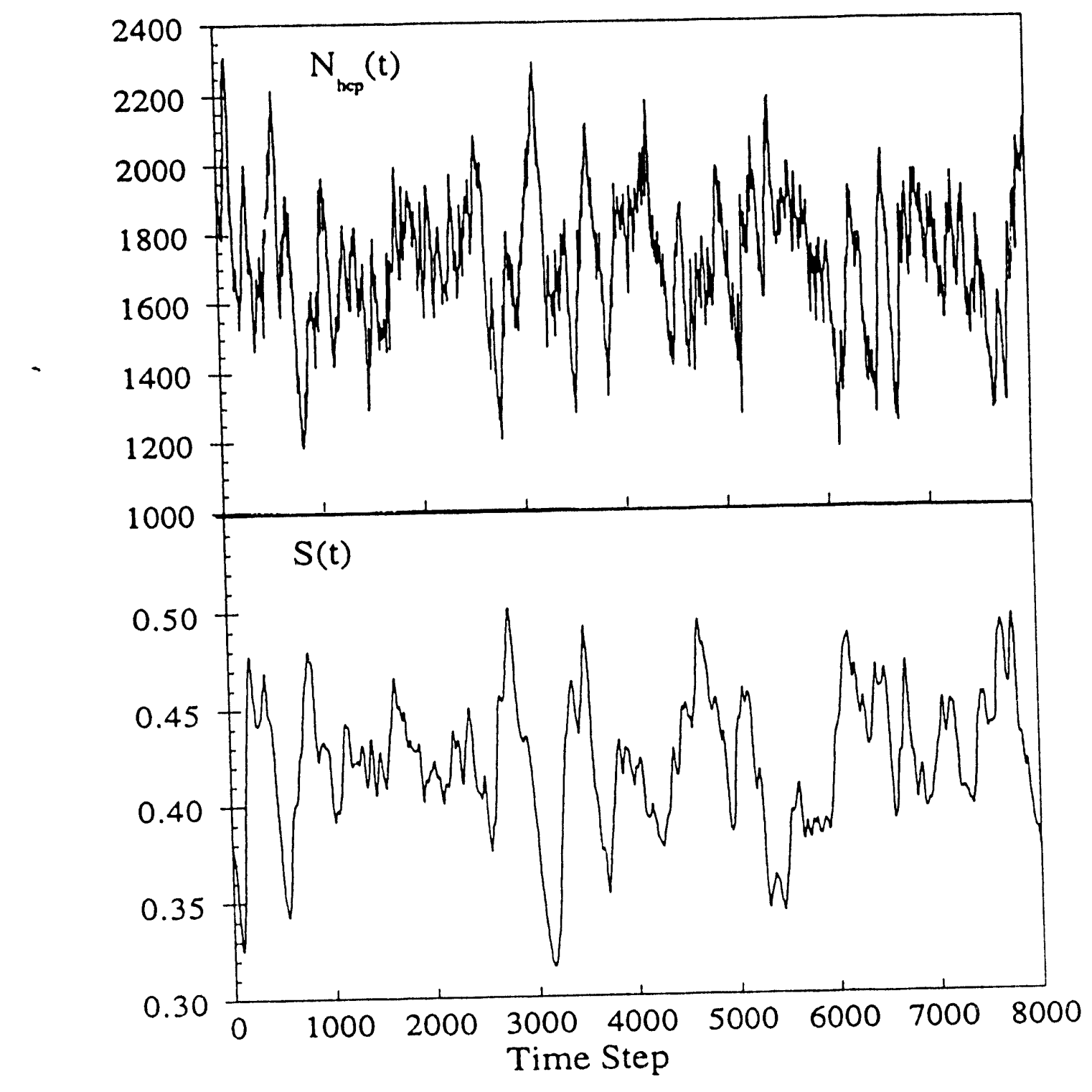

Figure 4.6: Time evolutions of (a) $S(\mathrm{t})$ and (b) $N_{h c p}(\mathrm{t})$. 


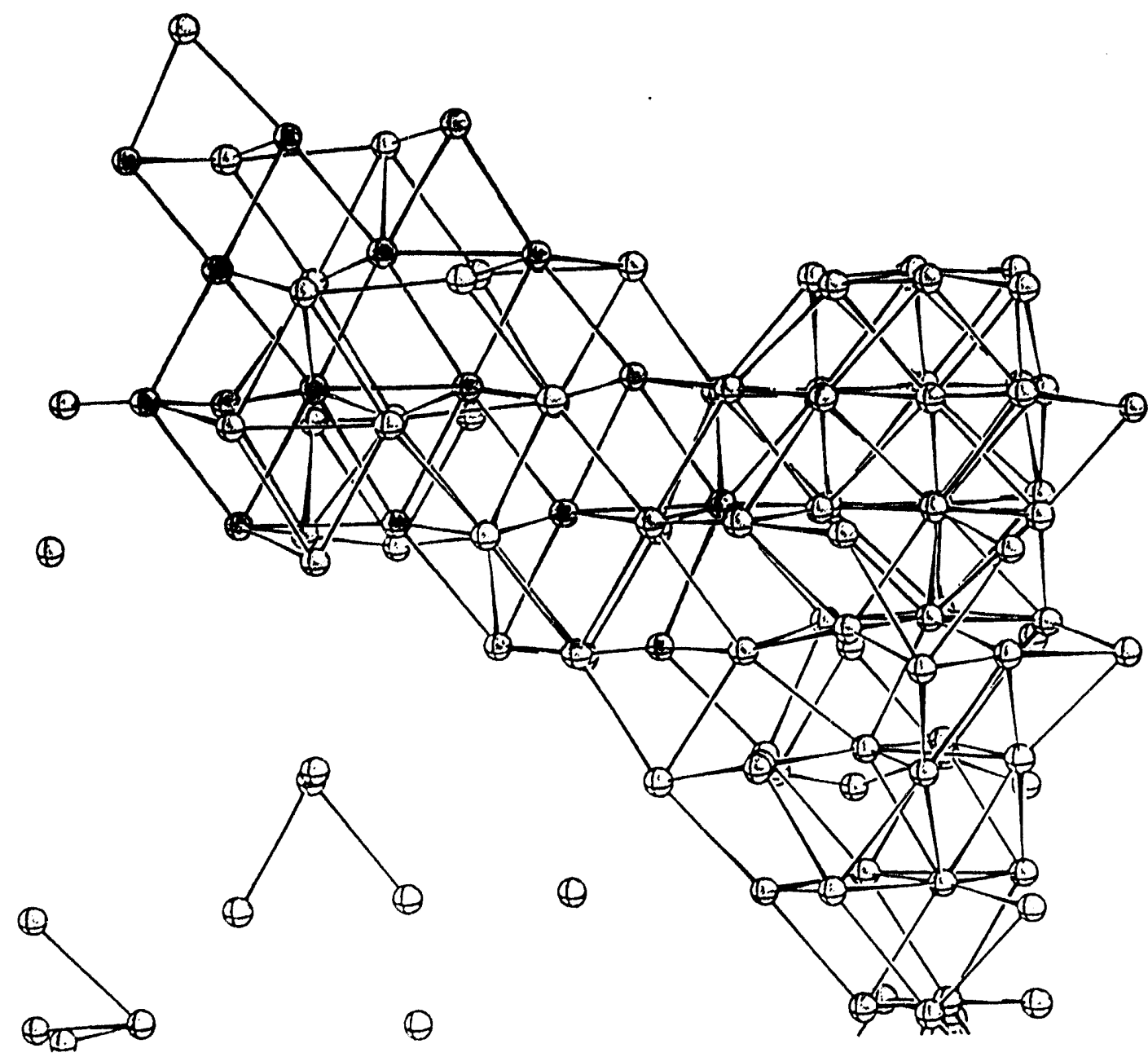

Figure 4.7: The snapshot picture of atomic configuration at an instant when $N_{h c p}$ has a peak (Fig. 4.6). Only part of the atoms are shown to facilitate the view of these hcp "embryos". 
space observed in experiments $[4,11]$ and reproduced by our calculations. We note that the strong asymmetry between the scattering intensities at $\mathbf{A}=\left(\frac{7}{3}, \frac{7}{3}, \frac{4}{3}\right)$ and at $\mathbf{B}=\left(\frac{5}{3}, \frac{5}{3}, \frac{8}{3}\right)$ can not be explained by simple anharmonicity or phase fluctuation arguments. Since the magnitudes of these two wavevectors are the same, random thermal vibration should not favour one over the other. Also, since these two $\mathbf{Q}$ points are both reciprocal lattices in the $h c p$ phase $((1, \overline{3}, 0)$ and $(2, \overline{3}, 0))$ with the same structure factors $\left(F^{2}=4\right)$, the asymmetry can not be explained by additional scattering from perfect $h c p$ regions in the bcc crystal either. From snapshots of the atomic positions, we find that, because of the dynamical fluctuations of the system, atoms are most likely to be found somewhere between the bcc and hcp configurations. In the $h c p$-like embryos, the atoms are not positioned at the center of the triangles of the atoms on the next plane as they should be in the perfect $h c p$ lattice, but shifted more often towards the sides of the triangle - their positions in the bcc structure. In going from the $b c c$ to the $h c p$ structure, the geometric structure factor can be expressed as

$$
F^{2}=\left|\sum_{i=1,2} \exp \left(i \mathbf{q} \cdot r_{i}\right)\right|^{2}=2(1+\cos (\theta))
$$

where,

$$
\theta=2 \pi\left(\sqrt{3} \delta H+\frac{2 \sqrt{3}}{3} \delta K+\frac{1}{2} L+\frac{1}{2} H\right) .
$$

Here, $[H, K, L]$ are $[1, \overline{3}, 0]$ and $[2, \overline{3}, 0]$ respectively for $\mathbf{A}$ and $\mathbf{B}$, and $\delta$ is the displacement away from the ideal bcc position (shown in Fig. 4.1). The transition is completed when $\delta=\frac{\sqrt{3}}{6}$. The geometric structure factor is pluited as a function of $\delta$ in Fig. 4.8. The intensity at point $\mathbf{A}$ (strong $\omega$ ) is much stronger than that of point B (weak $\omega$ ) when the system is midway between the $h c p$ and the bcc structure. Also, in accordance with the Burgers mechanism [75], the increase in $\delta$ is accompanied by 
a uniform shear of the lattice, leading to the streaking of the quasi-elastic scattering contours in the $[11 \overline{2}]$ directions as shown in Fig. 4.5.

For the above calculations, we have tested the unit cell size effect and the differences between the two potential models. Since the stability of the bcc phase is achieved by the constant-volume constraint in our simulation, a larger unit cell will introduce more low frequency phonon modes and enhance the instability of the bcc. This is manifested in a stronger asymmetry in the phonon spectra of $\mathbf{A}=\left(\frac{7}{3}, \frac{7}{3}, \frac{4}{3}\right)$ and $\mathbf{B}=\left(\frac{5}{3}, \frac{5}{3}, \frac{8}{3}\right)$ points and stronger quasi-elastic intensity at $\frac{4}{3}[1,1,1]$ as the unit cell size increases. If the unit cell is too large, 6750 atoms for EAM2 and 11664 atoms for EAM1, the $b c c$ lattice is no longer stable. In these cases, the systems do not transform to the low temperature $h c p$ phase due to the restriction on the shape of the unit cell. From Figs. 3.1 and 3.2, it is obvious that bcc phase is more stable for the EAM1 potential than for the EAM2 potential since the $\mathrm{T}_{1}$ modes along $\xi \xi \xi 0$ ! become unstable for EAM2. However, the basic features do not change much as long as the systems still stay in the bcc structure. This indicates that phase fluctuations and the anomalous phonon behavior are not very senstive to the details of the potential model. Thus, the anomalous phonon behavior is expected to be generic for a series of materials as observed in experiments $[19,20,21,22,23]$.

\section{Conclusion}

In summary, using molecular dynamics, we have simulated phase fluctuations in $b c c \mathrm{Z}_{\mathrm{r}}$ at high temperatures. The neutron scattering contour and phonon spectra obtained from our simulation agree very well with experimental observations. The weak dependence of the potential models indicates that this kind of phenomenon 


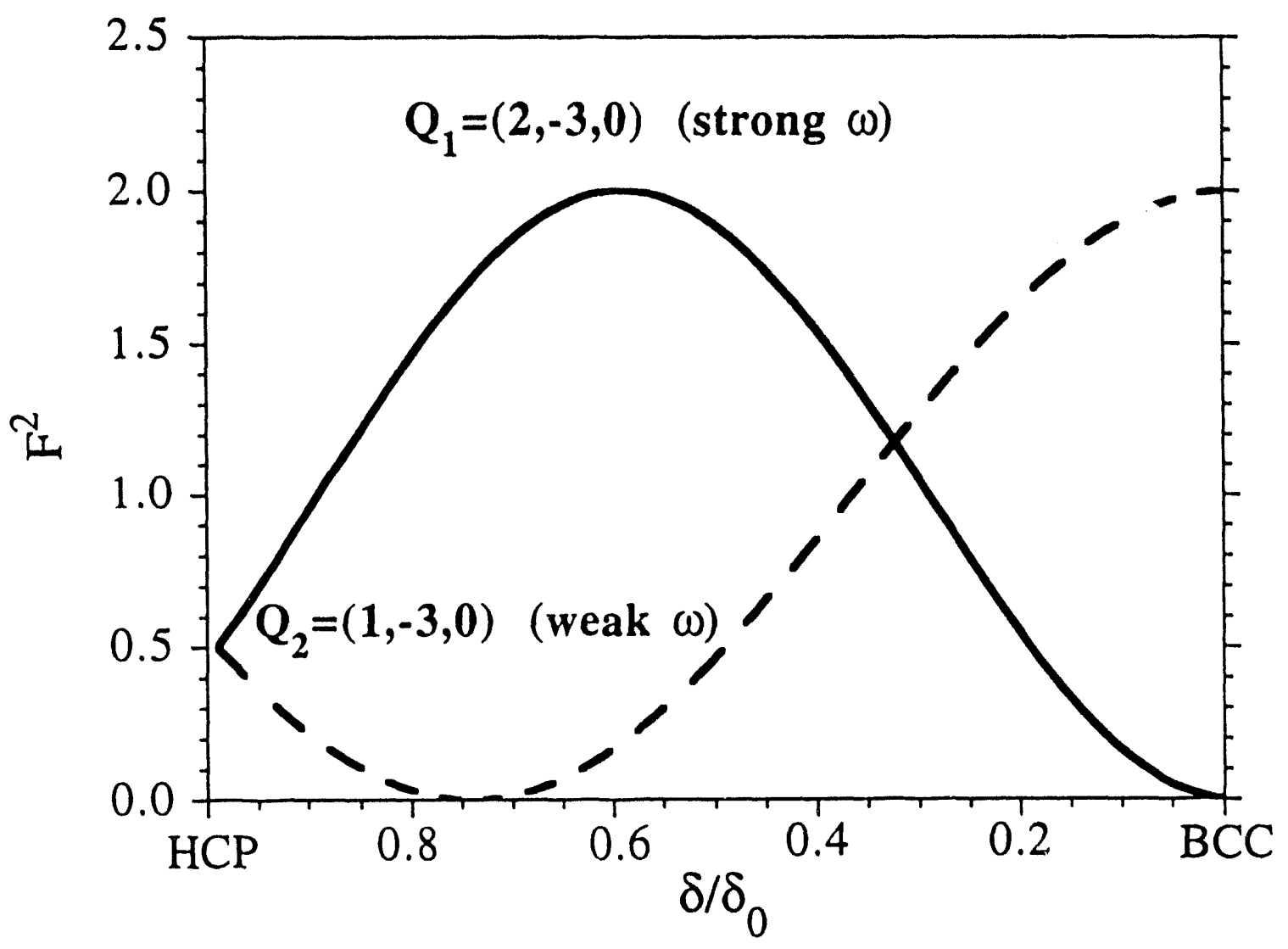

Figure 4.8: Geometric structure factors of strong and weak $\omega$ points along the bcc to $h c p$ transformation path. 
is generic for a wide range of $b c c$ materials that undergo phase transformations to another structure at low temperatures. However, there are still a number of open questions. One is the role of defects and stoichiometric variations in pinning the phase fluctuations in quenched alloys. Another is the role of $b c c$ to $\omega$ phase fluctuations. Due to the limitation of the classical potential employed in the calculation, the energy of the $\omega$ phase in our model is $0.1 \mathrm{eV}$ per atom higher than that of the first-principles calcr'ations [12]. Thus, possible fluctuations into the $\omega$ phase are suppressed in our calcu. ions. One of the consequences is reduced quasi-elastic scattering and a welldefined, honon spectrum around $\mathbf{Q}=\frac{2}{3}(1,1,1)$ (Fig. 4.2). We note that preliminary data [81] a pure bcc $\mathrm{Z}_{\mathrm{r}}$ at high temperature seem to support our result. For the quenched alloy systems, Mössbauer and X-ray $[7 \mid$ measurements as a function of alloy concentrations indicated that, while the strong quasi-elastic peak around $\mathbf{Q}=\frac{2}{3}(1,1,1)$ is well-defined for low $\mathrm{Nb}$ concentrations (less than 10\%), the quasi-elastic scattering becomes diffuse at large $\mathrm{Nb}$ concentrations. Our results provide a good description of the observed scattering at large $\mathrm{Nb}$ concentrations and at high temperatures. Further investigations of the phonon spectra at $\mathbf{Q}=\frac{2}{3}(1,1,1)$ as a function of alloy concentrations would be very useful for understanding the development of $\omega$ phase fluctuations with alloying. 


\section{CHAPTER 5. TIGHT-BINDING MOLECULAR DYNAMICS}

\section{Concepts and Applications in Carbon System}

Despite the great success of the EAM potential for close-packed metal systems, the interatomic interactions in semiconductor materials are hard to describe by classical potentials which have two major shortages: Firstly, unlike metals, semiconductor materials have strong directional bonds between atoms. Secondly, the classical potentials have no electronic band structure information which is very important in semiconductor materials. While first-principles calculations are much more accurate, their application in dynamic simulations is still limited by the huge computational cost.

Since the pioneering work of Chadi [82], the empirical tight-binding (TB) model has been widely applied to the studies of semiconductor systems $[83,84,85,86,87]$. In these schemes, the TB parameters are determined by fitting to an accurate firstprinciple electronic band structure calculation. The tight-binding scheme aims at modeling the covalent bonding in semiconducting systems through the incorporation of the electronic structure which is calculated for each atomic configuration with an empirical tight-binding Hamiltonian. This scheme tries to include the essential quantum mechanics of atomic bonding in the system while still keeping the computational effort small enough that large systems and long simulation periods can be studied. 
The transferability of the TB parameters is enhanced by fitting to various crystal structures with a large range of interatomic distances.

While the TB electronic band description is not as accurate as tie first-principles band structure, its efficiency and comparative accuracy allow it to serve as a bridge between the classical potentials and the first-principles calculations.

The total energy of a system of electrons and ions can be written as

$$
H=T_{e}+T_{i}+V_{i-i}+V_{e-e}(r)+V_{i-e}(\{r\},\{R\})
$$

where $T_{e}$ is the electronic kinetic energy, $T_{i}$ is the ionic kinetic energy, $V_{i-i}$ is the ion-ion interaction energy, $V_{e-e}$ is the electron-electron interaction energy, and $V_{i-e}$ is the ion-electron interaction. Here, $\{r\}$ and $\{R\}$ denotes the electronic coordinates and ionic coordinates respectively. With the usual Born-Oppenheimer approximation between the electrons and ions, the total energy can be written as

$$
E_{\text {total }}(\{R\})=I_{i-i}(\{R\})+E_{e}(\{R\})
$$

where $E_{e}$ is the total electronic energy which depends parametrically on the ionic configuration $\{R\}$. The tight-binding scheme attempts to express $E_{e}$ in terms of eigenvalues of the one-electron Hamiltonian

$$
\left(T_{e}+V_{i-i}+V_{e-e}\right) \psi_{n \mathbf{k}}=\varepsilon_{n \mathbf{k}} \psi_{n \mathbf{k}}
$$

where $\mathrm{n}$ is the band index, and the $\mathbf{k}$ is the wave vector. Then the band structure energy is

$$
E_{b s}=\sum_{n \mathbf{k}} \varepsilon_{n \mathbf{k}} f_{n \mathbf{k}}
$$

where $f_{n \mathbf{k}}$ is the Fermi-Dirac distribution function. In the above expression, the twobody electron-electron interaction terms have been included as a one-body effective 
potential. Therefore, $E_{b s}$ includes the electron-electron interaction twice, and this double counting can be corrected by defining:

$$
U_{0}(\{R\})=V_{i_{-i}}(\{R\})-<V_{e-e}(\{R\})>
$$

where $<>$ denotes the expectation value. This arrangement has the advantage that for two ions that are separated by a distance much larger than the screening length that combined effect of the ion and the screening electron system is nearly neutral, making $U_{0}(\{R\})$ quite short ranged, which is very important for the efficiency of a calculation. The total energy is written as

$$
E_{\text {total }}(\{R\})=U_{0}(\{R\})+E_{b s}(\{R\})
$$

where

$$
U_{0}(\{R\})=\sum_{i, j} \phi_{i, j}
$$

is the two-body potential energy. $U_{0}(\{R\})$ is determined by subtracting $E_{b s}$ from $E_{\text {total }}$ provided the latter is known either from the first-principles total energy calculations or from experiments. The atoms are subject to two kinds of forces, the two-body force can be obtained from the derivation of the two-body potential while the force arising from band structure can be calculated by the Hellinann-Feynman theorem

$$
F_{i}^{H F}=-\sum_{n \mathbf{k}} f_{n \mathbf{k}}<\psi_{n \mathbf{k}}\left|\frac{\partial H}{\partial R_{i}}\right| \psi>,
$$

where $H$ is the TB Hamiltonian. The TB potential model used in this study is from the work of $\mathrm{Xu}$ et al. [41]. The total energy is expressed as

$$
E_{\text {total }}\left(\left\{R_{i}\right\}\right)=\sum_{n}^{\text {occupied }}<\psi_{n}\left|H_{T B}\left(\left\{R_{i}\right\}\right)\right| \psi_{n}>+E_{r e p}\left(\left\{R_{i}\right\}\right)
$$


The orthogonal $s p^{3}$ basis TB Hamiltonian $H_{T B}\left(\left\{R_{i}\right\}\right)$ is described by the following parameters: $\varepsilon_{s}$ and $\varepsilon_{p}$ are on-site atomic energies, $v_{s s}\left(R_{i, j}\right), v_{s p \sigma}\left(R_{i, j}\right)$, $v_{p p \sigma}\left(R_{i, j}\right)$ and $v_{p p \pi}\left(R_{i, j}\right)$ are overlap parameters which decay rapidly with interatomic distance $R_{i, j}$. The repulsive energy is in the form of

$$
E_{r e p}=\sum_{i} f\left[\sum_{j \neq i} \phi\left(R_{i, j}\right)\right]
$$

where $\phi\left(R_{i, j}\right)$ is a pairwise interaction and $f$ is a function with argument $\sum_{j} \phi\left(R_{i, j}\right)$. The above parameters and functions are fitted to the first-principles results for electronic band structures and binding energies of graphite, diamond and a linear carbon chain as a function of the carbon-carbon bond length. This model not only reproduces the binding energies of structures with different coordination numbers, graphite (3), diamond (4) and linear chain (2), but also describes well the properties of the liquid carbon state. The reliability of this potential for cluster calculations is tested for small clusters: In the range $5 \leq n \leq 11$, odd-numbered clusters prefer a linear structure, while even-numbered clusters prefer a ring structure, in good agreement with $a b$ initio quantum mechanical calculations 88 . For bigger clusters, the present TB calculation on $\mathrm{C}_{60}$ yields a heat of formation of $0.40 \mathrm{eV}$ per atom (with respect to that of graphite) and a HOMO-LUMO energy gap of $1.60 \mathrm{eV}$. These values agree quite well with the $a b$ initio local density approximation (LDA) results of $0.39 \mathrm{eV}$ per atom and $1.71 \mathrm{eV}$ respectively 89$]$. Since this potential was fitted for bulk carbon, we expect that it will be even more reliable as we extend our calculation to larger clusters. 


\section{Implementation of Electronic Temperature}

For the TB potential model, the molecular dynamics simulation is performed in the same fashion as described in Chapter 2. The expressions for the band structure energy and Hellman-Feynman force in Equations 5.8 and 5.9 are valid only for zero electronic temperature. However, in some cases, we need to simulate high temperature situations in which the electronic temperature can no longer be regarded as zero. To include the effects of finite temperature on the electronic states, we used the Fermi-Dirac (F-D) distribution to describe the occupation of the electronic states in the energy and force calculations in our tight-binding molecular dynamics (TBMD) simulations. Our procedure follows closely those used in recent first-principles molecular dynamics (FPMD) studies of metallic systems $[90,91,92]$. It has been observed that the inclusion of electronic temperature effects not only avoids the instability caused by changing occupancies of states near the Fermi level in metallic systems, but also includes the electronic entropy into the calculation in a convenient manner.

The general expressions of band structure energy and Hellman-Feynman force are

$$
\begin{gathered}
E_{T B}=2 \sum_{i} \varepsilon_{i} f_{i} \\
F_{\alpha}=2 \sum_{i}<\psi_{i}\left|\nabla_{\alpha} H_{T B}\right| \psi_{i}>f_{i}
\end{gathered}
$$

where,

$$
f_{i}=\frac{1}{e^{\left(\varepsilon_{i}-\mu\right) / k_{B} T_{e l}+1}} .
$$

The $\mu$ is the chemical potential and it is adjusted every time step in the simulation 
to guarantee the conservation of the total number of electrons

$$
2 \sum_{i} f_{i}=N_{\text {ele. }}
$$

However in the first-principles molecular dynamics (FPMD), it was noted by Pederson and Jackson [91] that to conserve the total dynamical energy $\mathrm{U}=\mathrm{K}_{I}+\mathrm{E}_{\text {total }}$, one must also consider the changes of the occupancy number $f_{i}$. The force obtained from Equation 5.12 misses one term

$$
\sum_{i} \frac{\partial E_{T B}}{\partial f_{i}} \frac{\partial f_{i}}{\partial\left(\varepsilon_{i}-\mu\right)} \nabla_{\alpha}\left(\varepsilon_{i}-\mu\right)
$$

and unfortunately this term is very difficult to calculate. Later, Wentzcovitch, Martin and Allen [92] introduced the Mermin free energy [93]

$$
\Omega=E_{\text {total }}+K_{I}-T_{e l} S
$$

where,

$$
S=-2 k_{B} \sum_{i}\left[f_{i} \ln f_{i}+\left(1-f_{i}\right) \ln \left(1-f_{i}\right)\right]
$$

and they showed numerically that the free energy $\Omega$ is conserved by using forces calculated from 5.12. In the TBMD simulation, also in FPMD, we can prove analytically that the expression 5.12 is indeed the real "force" if one includes electronic entropy effects. The dropped term in Equation 5.15 is cancelled out by the derivative of the electronic entropy

$$
\frac{\partial\left(T_{e l} S\right)}{\partial \alpha}=T_{e l} \cdot \sum_{i} \frac{\partial S}{\partial f_{i}} \cdot \frac{\partial f_{i}}{\partial \varepsilon_{i}} \cdot \nabla_{\alpha} \varepsilon_{i}
$$

Equation 5.18 can be rewritten as

$$
-2 k_{B} T_{e l} \sum_{i} \cdot \frac{\partial\left[f_{i} \ln f_{i}+\left(1-f_{i}\right) \ln \left(1-f_{i}\right)\right]}{\partial f_{i}} \cdot \frac{\partial f_{i}}{\partial\left(\varepsilon_{i}-\varepsilon_{f}\right)} \cdot \nabla \alpha\left(\varepsilon_{i}-\varepsilon_{f}\right),
$$


and after some simple algebraic manipulations, Equation 5.19 can be simplified to

$$
2 \sum_{i} \varepsilon_{i} \cdot \frac{\partial f_{i}}{\partial\left(\varepsilon_{i}-\varepsilon_{f}\right)} \cdot \nabla_{\alpha}\left(\varepsilon_{i}-\varepsilon_{f}\right)
$$

Here we used the conservation of the total number of electrons

$$
\varepsilon_{f} \sum_{i} \frac{\partial f_{i}}{\partial\left(\varepsilon_{i}-\varepsilon_{f}\right)} \cdot \nabla_{\alpha}\left(\varepsilon_{i}-\varepsilon_{f}\right)=\frac{\partial}{\partial \alpha} \sum_{i} f_{i}=\frac{\partial}{\partial \alpha} N_{e l e}=0
$$

From Equation 5.11

$$
\frac{\partial E_{T B}}{\partial f_{i}}=2 \varepsilon_{i}
$$

Then it is obvious that 5.15 is cancelled out by the derivative of electronic entropy 5.18. The force we calculate from Equation 5.12 is

$$
F_{\alpha}=-\nabla \alpha \Omega
$$

The above implementation of Fermi-Dirac distribution function is not necessary for zero temperature optimization and is not important for semiconductor systems as long as the gaps are relatively large compared with the temperature. But its importance appears in the metallic systems and semiconductor systems at high temperature. In the Chapter 6 , we will test this implementation and discuss its effects on thermal disintegration of carbon clusters. 
CHAPTER 6. STRUCTURES AND STABILITIES OF FULLERENE

\section{Introduction}

Though the dominant products extracted from the evaporation of graphite are $\mathrm{C}_{60}$ and $\mathrm{C}_{70}$, there are minor admixtures of other fullerenes $[42,43,44,45,46]$. Because these fullerenes have their own unique structures and potential applications, there are intense efforts to isolate them and to characterize their chemical and physical properties. One important piece of experimental evidence for the cluster structure comes from the ${ }^{13} \mathrm{C}$ nuclear magnetic resonance (NMR) spectra of the molecules. The chemical shift measured by NMR is determined by the environment of each atom so the atoms equivalent with each other by symmetry operations have same chemical shift. From the number and intensity of NMR peaks, it is possible in some cases $[34,43]$ to determine the symmetry of the molecule. For example, all the atoms in the $\mathrm{C}_{60}$ molecule are equivalent by symmetry and the measured NMR spectrum shows a single peak [34]. However, it is hard to pinpoint the symmetry exactly when the molecular structure has a low symmetry. Even when the symmetry is known, the structure is still undermined because some fullerene can have more than one structure with the same symmetry.

On the theoretical side, existing approaches can be roughly divided into two categories. In the first approach, educated guesses based mostly on symmetry considera- 
tions were used to construct plausible topological networks $\{94,95,96,97,98,99,100\}$, the ground-state structure is then selected by comparing the total energy or simply the highest occupied molecular orbit-lowest unoccupied molecular orbit (HOMOLUMO) gaps of the various candidates. Such schemes are useful only for clusters of certain special sizes and the deduced structures usually possess high symmetry. But the recently isolated $\mathrm{C}_{76}[43], \mathrm{C}_{84}[46]$ and $\mathrm{C}_{78}[42,46]$ all have relatively low: symmetry structures. To overcome this limitation, Manolopoulos [97] introduced a new geometrical method which uses the computer to search for every topologically distinct network. Since there is no symmetry constraint, the number of possible isomers is huge. For example, there are 1812 distinct isomers for $\mathrm{C}_{60}$ [97]. One useful empirical rule is the isolated-pentagon rule which states that fullerenes prefer those isomers which have no touching pentagons. Since the curvature of the cage structures is provided by pentagons, isolated-pentagon isomers distribute curvature more uniformly to minimize the surface tension. The isolated-pentagon rule is very effective for clusters between 60 and 70 . For example, $\mathrm{C}_{60}$ only has one isolatedpentagon isomer and it is indeed the ground-state icosahedral structure. But the number of isolated-pentagon isomers grows very fast for clusters larger than $\mathrm{C}_{80}$ and the problem becomes quite intractable for fullerenes beyond $\mathrm{C}_{90}$ [101]. The numbers of isolated-pentagon isomers for large fullerenes are listed in Table 6.1. More seriously, for clusters from $\mathrm{C}_{62}$ to $\mathrm{C}_{68}$ and for clusters smaller than $\mathrm{C}_{60}$, there exist no isolated-pentagon isomers. Then the ring-spiral algorithm produces too many candidates to be checked by $a b$ initio calculations, while the simple potential models are not accurate enough to determine the ground-state geometries [101].

In the second approach, the ground-state structures are obtained by simulated 
Table 6.1: Enumeration of spectrally distinct isolated-pentagon $\mathrm{C}_{n}$ fullerene isomers found by the ring spiral algorithm [101] in the range $n=70-100$.

\begin{tabular}{cccccccc}
\hline \hline $\mathrm{n}$ & Isomers & $\mathrm{n}$ & Isomers & $\mathrm{n}$ & Isomers & $\mathrm{n}$ & Isomers \\
\cline { 3 - 8 } 70 & 1 & 78 & 5 & 86 & 19 & 94 & 134 \\
\hline 72 & 1 & 80 & 7 & 88 & 35 & 96 & 187 \\
\hline 74 & 1 & 82 & 9 & 90 & 46 & 98 & 259 \\
\hline 76 & 2 & 84 & 24 & 92 & 86 & 100 & 450 \\
\hline \hline
\end{tabular}

annealing $[131,102,103]$. This approach requires an accurate and efficient interatomic potential. It is still beyond our present computer capability to perform first-principles molecular dynamics for large fullerenes over tens of thousands of steps. A more serious problem is that the system will often be trapped in metastable high-energy structures because the strong directional bonding of carbon creates large energy barriers between various metastable structures and the ground-state structure. For example, in the work of Ballone et al. [131], it was found that structures of $\mathrm{C}_{62}$ and $\mathrm{C}_{64}$ always contain seven-member ring defects although later we found many more stable isomers for these clusters with five- and six-member rings only. The works of $\mathrm{Xu}$ et al. [103] and Chelikowsky [102] also failed to produce a perfect $I_{h}$ ground-state structure for $\mathrm{C}_{60}$, although they were able to get a cage structure for $\mathrm{C}_{60}$ from their simulated annealing. Since the energy required to break carbon-carbon bonds and to rearrange the network is so high there is little chance to reach the ground-state structure starting from a random initial configuration. Simulated annealing will be useful only if one can start the simulation from an initial structure which already has the correct interatomic bond network. 


\section{Ground-State Structure Search Scheme}

To overcome the difficulties mentioned above, we devised a scheme for generating energetically favorable structures for fullerenes [47]. In this scheme, we first try to generate the correct topological networks for energetically favourable structures. Instead of looking at the network connecting individual atoms, we focus our attention on the "face-dual" network obtained by linking the centers of each polygonal face of the cage structure. Since each atom of the fullerene is threefold coordinated, the face-dual network consists of a triangular mesh. In comparison with the original atomic network, the triangle mesh of the face-dual network is much easy to generate and anneal. Thus we divide the search for the ground-state structure for fullerene into two steps: first, a simulated annealing scheme is used to search for energetically favourable face-dual triangular networks which are then inverted to pick up a short list of candidate fullerene structures. After this, the ground-state structure is determined by selecting the lowest-energy geometry obtained from unconstrained optimization of the atomic positions for each of the candidate networks using TBMD.

To obtain the energetically favourable face-dual networks, we represent each polygon of a fullerene by a point lying on a predetermined closed surface. An artificial two-body repulsive interaction is introduced between these points. The total energy of the system is expressed as

$$
E_{\text {total }}=\frac{1}{2} \sum_{i, j} \frac{a}{r_{i j}^{n}}+b\left(\sum_{i}\left(\left(x_{i}-1\right)^{2}+\left(y_{i}-l_{y}\right)^{2}+\left(z_{i}-l_{z}\right)^{2}\right)+\frac{1}{2} \sum_{i} m_{i} v_{i}^{2}\right.
$$

where the first term is the repulsive interaction and the second term is the potential well to confine all the points on the ellipsoidal surface. The parameters $m_{i}$ and $v_{i}$ are the artificial mass and velocity of each face point. The parameters $l_{y}$ and $l_{z}$ can be 
adjusted to change the shape of the surface. The simulated annealing is performed by first heating the system up to $1000 \mathrm{~K}$. After $20000 \mathrm{MD}$ steps for thermal equilibration at $1000 \mathrm{~K}$, the system is then cooled down to $0 \mathrm{~K}$. The resultant network obtained by this procedure is a triangular mesh spanning the original surface, which can be inverted to obtain the atomic network. In this scheme, whether a face is pentagonal or hexagonal is determined by the number of neighboring faces. The use of the face-dual network rather than the atomic network for simulated annealing has the advantage that the change from pentagon to hexagon can occur easily with a small energy barrier $[105,106]$. Even for quite large number of atoms, the system will not be trapped in local metastable states. The repulsive potential acts to separate the pentagonal faces as far apart as possible. It also tends to place the pentagonal faces at the surface areas with sharp curvature. As a first test, we put 32 points, corresponding to $\mathrm{C}_{60}$, on the surface of a sphere and simulated according to the above procedure. The atomic network we obtained is exactly the $\mathrm{I}_{h}$ isomer of $\mathrm{C}_{60}$. To test the stability of this scheme, we change the initial configuration of face points as well as the force parameter and the outcomes are same.

The parameters that can be varied in this scheme are the shape of the predetermined surface and the range of the two-body repulsive potential. Although, in principle, the search should be performed for every possible constrained surface, we found that confining the face-dual network to ellipsoidal surfaces is already quite sufficient for fullerenes in the range from $C_{20}$ to $C_{100}$. Further symmetry breaking of the surface, if it is energetically favourable, can be realized in the following unconstrained tight-binding molecular dynamics optimization process. In the search for the energetically favourable face-dual network for each fullerene, we varied the 
ratio of the three axes of the ellipsoidal surface from 0.8 to 1.2 in steps of 0.05 . With these variations of the constrained surfaces, we obtained the two isolated-pentagon isomers of $\mathrm{C}_{76}$ and all five isolated-pentagon isomers of $\mathrm{C}_{78}$. For $\mathrm{C}_{84}$, we obtained 11 isomers (including the two lowest-energy $\mathrm{D}_{2}$ and $\mathrm{D}_{2 d}$ isomers) out of a total of 24 isolated-pentagon isomers for this fullerene [101]. When several isomers of a given fullerene are very close in shape, our scheme always picks up the one with the lowest energy. This selective nature turns out to be the advantage of the scheme, otherwise one has to examine each isomer to determine the ground-state structures of the fullerenes.

We found that the resultant face-dual networks also depend on the range of the repulsive two-body potential used in Equation 6.1 (although the dependence is very weak). In the study of $\mathrm{C}_{84}$, we found that if we fixed the constraining surface to a sphere and choose the two-body potential in the form of $\frac{1}{r^{n}}$, we obtained two distinct isomers with $\mathrm{n}=5$ and 6 respectively. When $n$ varies from 2 to 4 and from 7 to 12 , no new network was produced. In the rest of the study, we used both $\frac{1}{r^{5}}$ and $\frac{1}{r^{6}}$ for the repulsive interaction in the generation of the face-dual network for every case.

\section{Results and Discussion}

Using the scheme described above, we have systematically studied the structures of every even-number carbon clusters ranging from $C_{20}$ to $C_{100}$. A summary of our results for the heat of formation, symmetry, HOMO-LUMO gap and number of distinct NMR lines for low energy isomers is given in the Table 6.2.

Some interesting observations obtained from our calculations are discussed as follows: 
Table 6.2: Structural and electronic properties of carbon clusters. The values listed under Energy are the heat of formation of cluster relative to that of bulk graphite in the units of $\mathrm{eV}$ per atom. $\Delta \mathrm{E}$ is the energy of the isomer compared to that of the corresponding ground-state isome $i$ in units of $\mathrm{eV} /$ molecule. If there are two entries for the symmetry, the first one is topological symmetry and the second one is real symmetry due to Jahn-Teller distortion. The HOMO-LUMO energy separations are listed in the column HOMO-LUMO and the values in the column NMR are the numbers of distinct NMR lines expected.

\begin{tabular}{|c|c|c|c|c|c|}
\hline Cluster Size & Energy & $\Delta E$ & Symmetry & HOMO-LUMO & NMR \\
\hline 20 & 1.180 & & $\mathrm{I}_{h}\left(\mathrm{C}_{2}\right)$ & 0.015 & 10 \\
\hline 24 & 1.050 & & $\mathrm{D}_{6 d}$ & 0.448 & 2 \\
\hline 26 & 0.989 & & $\mathrm{C}_{2 v}\left(\mathrm{C}_{2}\right)$ & 0.292 & 13 \\
\hline 28 & 0.912 & & $\mathrm{~T}_{d}\left(\mathrm{C}_{1}\right)$ & 0.243 & 28 \\
\hline 30 & 0.850 & & $\mathrm{C}_{2 v}$ & 0.333 & 10 \\
\hline 32 & 0.781 & & $\mathrm{D}_{3}$ & 0.881 & 6 \\
\hline 34 & 0.758 & & $\mathrm{C}_{1}$ & 0.239 & 34 \\
\hline 36 & 0.706 & & $\mathrm{D}_{2 d}$ & 0.526 & 5 \\
\hline 38 & 0.673 & & $\mathrm{C}_{2}$ & 0.708 & 19 \\
\hline 40 & 0.641 & & $\mathrm{D}_{2}$ & 0.950 & 10 \\
\hline 42 & 0.614 & & $\mathrm{D}_{3}$ & 0.787 & 7 \\
\hline \multirow[t]{2}{*}{44} & 0.589 & & $\mathrm{D}_{2}$ & 0.732 & 11 \\
\hline & & 0.037 & $\mathrm{D}_{2}$ & 0.763 & 11 \\
\hline 46 & 0.573 & & $\mathrm{C}_{2}$ & 0.577 & 23 \\
\hline 48 & 0.552 & & $\mathrm{C}_{2}$ & 0.195 & 24 \\
\hline \multirow[t]{2}{*}{50} & 0.509 & & $\mathrm{D}_{5 h}$ & 0.503 & 4 \\
\hline & & 0.238 & $\mathrm{D}_{3}$ & 0.819 & 9 \\
\hline 52 & 0.502 & & $\mathrm{C}_{2}$ & 0.657 & 26 \\
\hline 54 & 0.482 & & $\mathrm{C}_{2 v}$ & 0.412 & 16 \\
\hline 56 & 0.467 & & $\mathrm{D}_{2}$ & 0.665 & 14 \\
\hline 58 & 0.453 & & $\mathrm{C}_{s}$ & 0.111 & 32 \\
\hline 60 & 0.401 & & $I_{h}$ & 1.61 & 1 \\
\hline
\end{tabular}




\begin{tabular}{|c|c|c|c|c|c|}
\hline Cluster Size & Energy & $\Delta \mathrm{E}$ & Symmetry & HOMO-LUMO & NMR \\
\hline 62 & 0.434 & & $\mathrm{C}_{2}$ & 0.378 & 31 \\
\hline 64 & 0.410 & & $\mathrm{D}_{2}$ & 0.952 & 16 \\
\hline 66 & 0.409 & & $\mathrm{C}_{2}$ & 0.471 & 33 \\
\hline 68 & 0.398 & & $\mathrm{C}_{2}$ & 0.725 & 34 \\
\hline 70 & 0.365 & & $\mathrm{D}_{5 h}$ & 1.103 & 5 \\
\hline 72 & 0.377 & & $\mathrm{D}_{6 d}$ & 1.388 & 4 \\
\hline 74 & 0.357 & & $\mathrm{D}_{3 h}$ & 0.224 & 9 \\
\hline \multirow[t]{2}{*}{$i 6$} & 0.354 & & $\mathrm{D}_{2}$ & 0.796 & 19 \\
\hline & & 0.242 & $\mathrm{~T}_{d}\left(\mathrm{D}_{2 d}\right)$ & 0.096 & 11 \\
\hline \multirow[t]{5}{*}{78} & 0.346 & & $\mathrm{C}_{2 v^{\prime}}$ & 0.493 & 22 \\
\hline & & 0.087 & $\mathrm{D}_{3 h^{\prime}}$ & 0.353 & 8 \\
\hline & & 0.284 & $\mathrm{C}_{2 v}$ & 0.545 & 21 \\
\hline & & 0.324 & $\mathrm{D}_{3}$ & 0.443 & 13 \\
\hline & & 0.913 & $D_{3 h}$ & 1.373 & 8 \\
\hline \multirow[t]{6}{*}{80} & 0.344 & & $\mathrm{D}_{5 h}\left(\mathrm{C}_{s}\right)$ & 0.076 & 40 \\
\hline & & 0.015 & $\mathrm{D}_{5 d}$ & 0.350 & 5 \\
\hline & & 0.030 & $\mathrm{C}_{2 v}$ & 0.198 & 22 \\
\hline & & 0.090 & $\mathrm{D}_{2}$ & 0.518 & 20 \\
\hline & & 0.165 & $\mathrm{C}_{2 v^{\prime}}$ & 0.141 & 23 \\
\hline & & 0.230 & $\mathrm{D}_{3}$ & 0.280 & 14 \\
\hline \multirow[t]{9}{*}{82} & 0.335 & & $\mathrm{C}_{2}$ & 0.649 & 41 \\
\hline & & 0.076 & $\mathrm{C}_{s}$ & 0.568 & 44 \\
\hline & & 0.166 & $\mathrm{C}_{2^{\prime}}$ & 0.362 & 41 \\
\hline & & 0.191 & $\mathrm{C}_{2^{\prime \prime}}$ & 0.477 & 41 \\
\hline & & 0.226 & $\mathrm{C}_{s^{\prime}}$ & 0.773 & 44 \\
\hline & & 0.229 & $\mathrm{C}_{s}{ }^{\prime \prime}$ & 0.234 & 44 \\
\hline & & 0.285 & $\mathrm{C}_{2 v}\left(\mathrm{C}_{2}\right)$ & 0.081 & 41 \\
\hline & & 0.566 & $\mathrm{C}_{3 v}$ & 0.120 & 17 \\
\hline & & 0.731 & $\mathrm{C}_{3 v^{\prime}}\left(\mathrm{C}_{s}\right)$ & 0.088 & 43 \\
\hline
\end{tabular}




\begin{tabular}{|c|c|c|c|c|c|}
\hline Cluster Size & Energy & $\Delta \mathrm{E}$ & Symmetry & HOMO-LUMO & NMR \\
\hline \multirow[t]{6}{*}{84} & 0.325 & & $\mathrm{D}_{2}(22)$ & 0.823 & 21 \\
\hline & & 0.033 & $\mathrm{D}_{2 d}(23)$ & 0.844 & 11 \\
\hline & & 0.309 & $\mathrm{D}_{6 h}(24)$ & 1.148 & 5 \\
\hline & & 0.434 & $\mathrm{D}_{2}(21)$ & 0.399 & 21 \\
\hline & & 0.741 & $\mathrm{D}_{2}(5)$ & 0.611 & 21 \\
\hline & & 1.231 & $\mathrm{D}_{2}(5)$ & 0.610 & 21 \\
\hline \multirow[t]{2}{*}{86} & 0.329 & & $\mathrm{C}_{1}$ & 0.344 & 86 \\
\hline & & 0.038 & $\mathrm{C}_{2}$ & 0.351 & 43 \\
\hline 88 & 0.327 & & $\mathrm{C}_{2}$ & 0.154 & 44 \\
\hline \multirow[t]{3}{*}{90} & 0.314 & & $\mathrm{C}_{2}$ & 0.633 & 45 \\
\hline & & 0.180 & $\mathrm{C}_{2 v}$ & 0.629 & 24 \\
\hline & & 0.307 & $\mathrm{C}_{2}$ & 0.564 & 45 \\
\hline \multirow[t]{2}{*}{92} & 0.311 & & $\mathrm{C}_{2}$ & 0.471 & 46 \\
\hline & & 0.071 & $\mathrm{D}_{2}$ & 0.575 & 23 \\
\hline 94 & 0.304 & & $\mathrm{C}_{2}$ & 0.679 & 47 \\
\hline \multirow[t]{4}{*}{96} & 0.300 & & $\mathrm{C}_{2}$ & 0.547 & 48 \\
\hline & & 0.320 & $\mathrm{C}_{1}$ & 0.630 & 96 \\
\hline & & 0.326 & $\mathrm{C}_{1}$ & 0.292 & 96 \\
\hline & & 0.355 & $\mathrm{D}_{6 d}$ & 0.318 & 5 \\
\hline \multirow[t]{2}{*}{98} & 0.300 & & $\mathrm{C}_{1}$ & 0.202 & 98 \\
\hline & & 0.054 & $\mathrm{C}_{s}$ & 0.606 & 50 \\
\hline \multirow[t]{2}{*}{100} & 0.292 & & $\mathrm{D}_{2}$ & 0.432 & 25 \\
\hline & & 0.602 & $\mathrm{D}_{5}\left(\mathrm{C}_{1}\right)$ & 0.062 & 100 \\
\hline
\end{tabular}


$\mathrm{C}_{20}\left(\mathrm{I}_{\mathrm{h}}\right)$
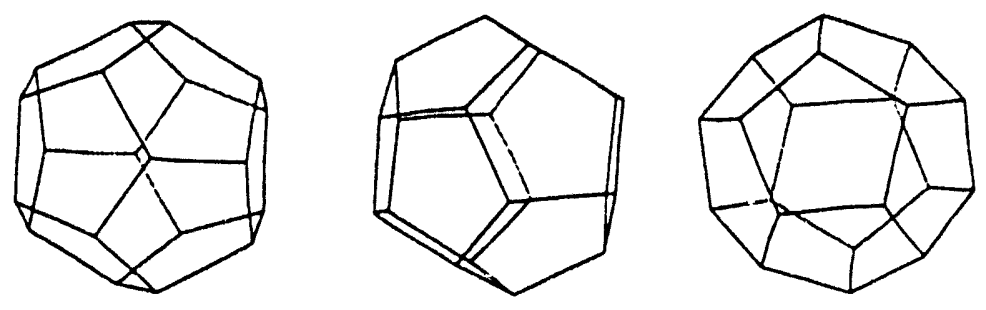

$\mathrm{C}_{24}$ (D6d)
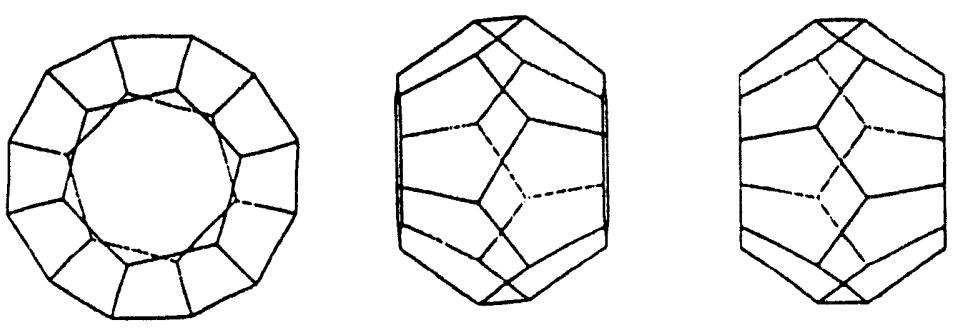

$\mathrm{C}_{26}\left(\mathrm{C}_{2 \mathrm{v}}\right)$
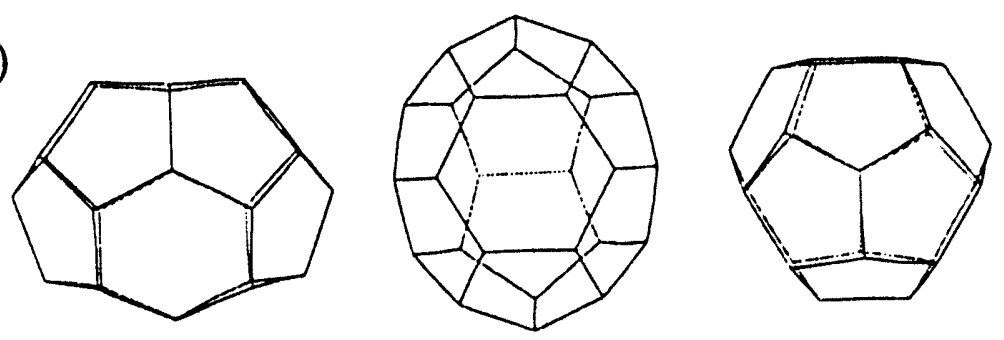

$\mathrm{C}_{28}\left(\mathrm{~T}_{\mathrm{d}}\right)$
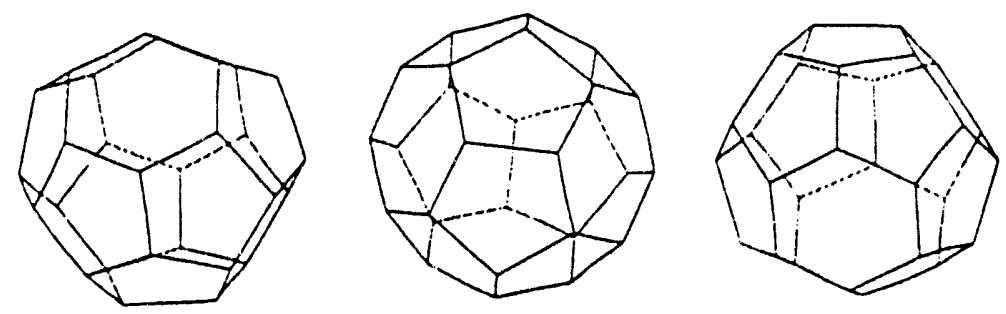

$\mathrm{C}_{30}\left(\mathrm{C}_{2 \mathrm{v}}\right)$
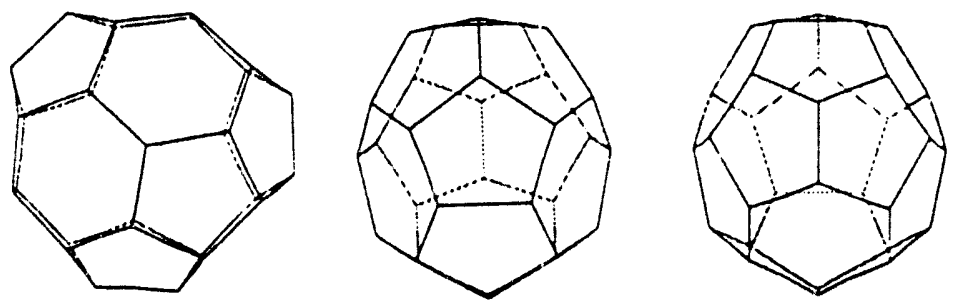

Figure 6.1: The line drawings of fully relaxed structures of fullerenes. The views along three ortliogonal axes are provided to appreciate the symmetries and the shapes of the cages. 
$\mathrm{C}_{32}$ (D3)
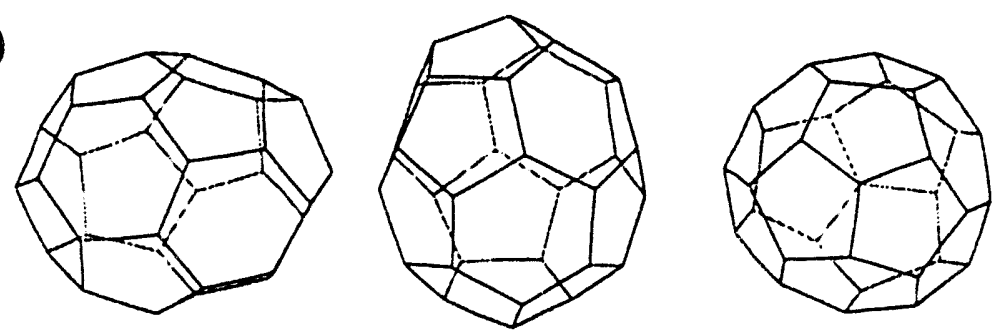

$\mathrm{C}_{34}\left(\mathrm{C}_{1}\right)$
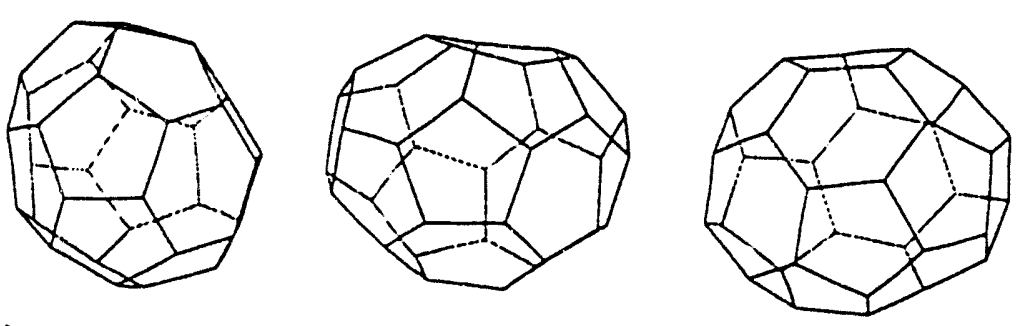

C36 (D2d)
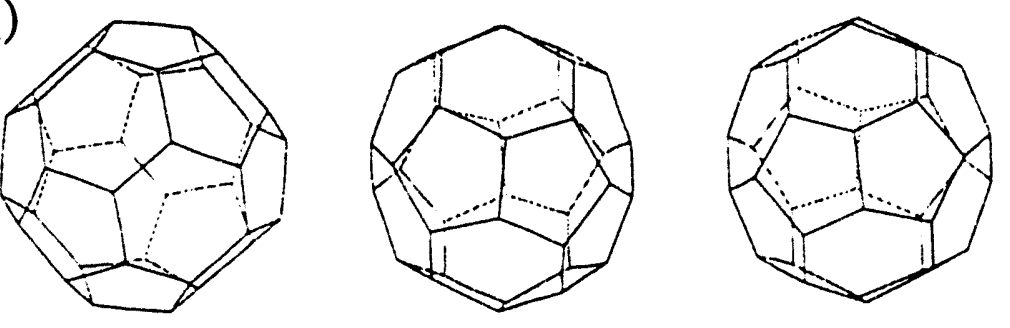

$\mathrm{C}_{38}\left(\mathrm{C}_{2}\right)$
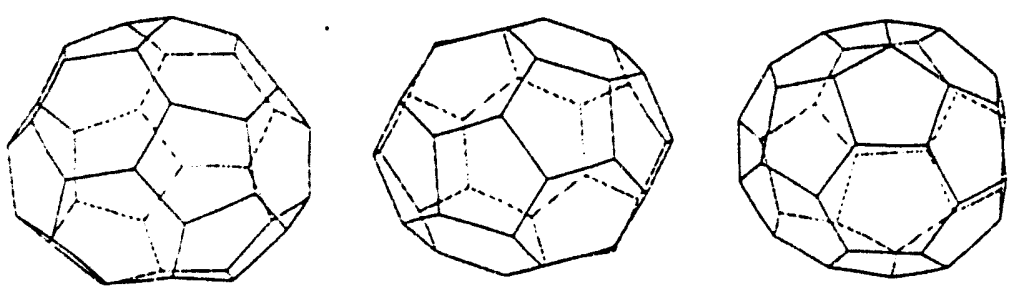

$\mathrm{C}_{40}\left(\mathrm{D}_{2}\right)$
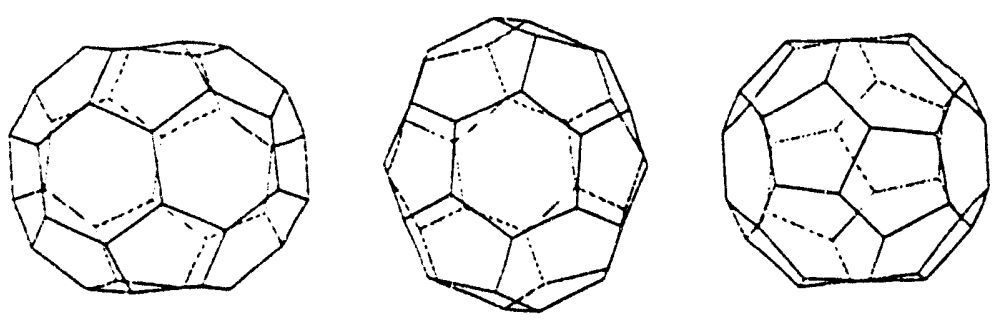

Figure 6.1 (Continued) 
$\mathrm{C}_{42}$ (D3)
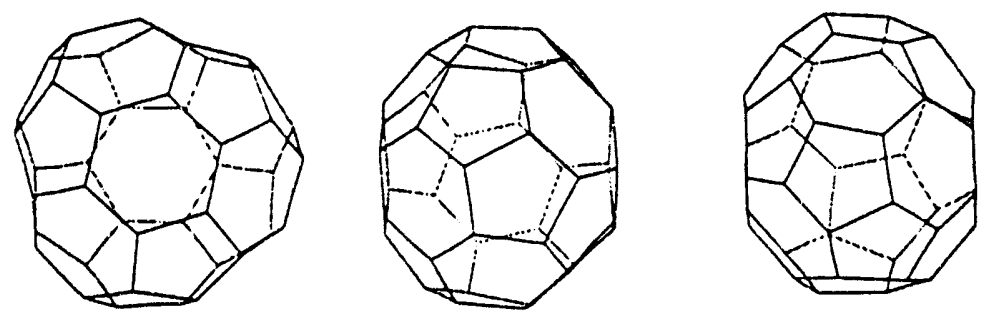

C44 (D2)
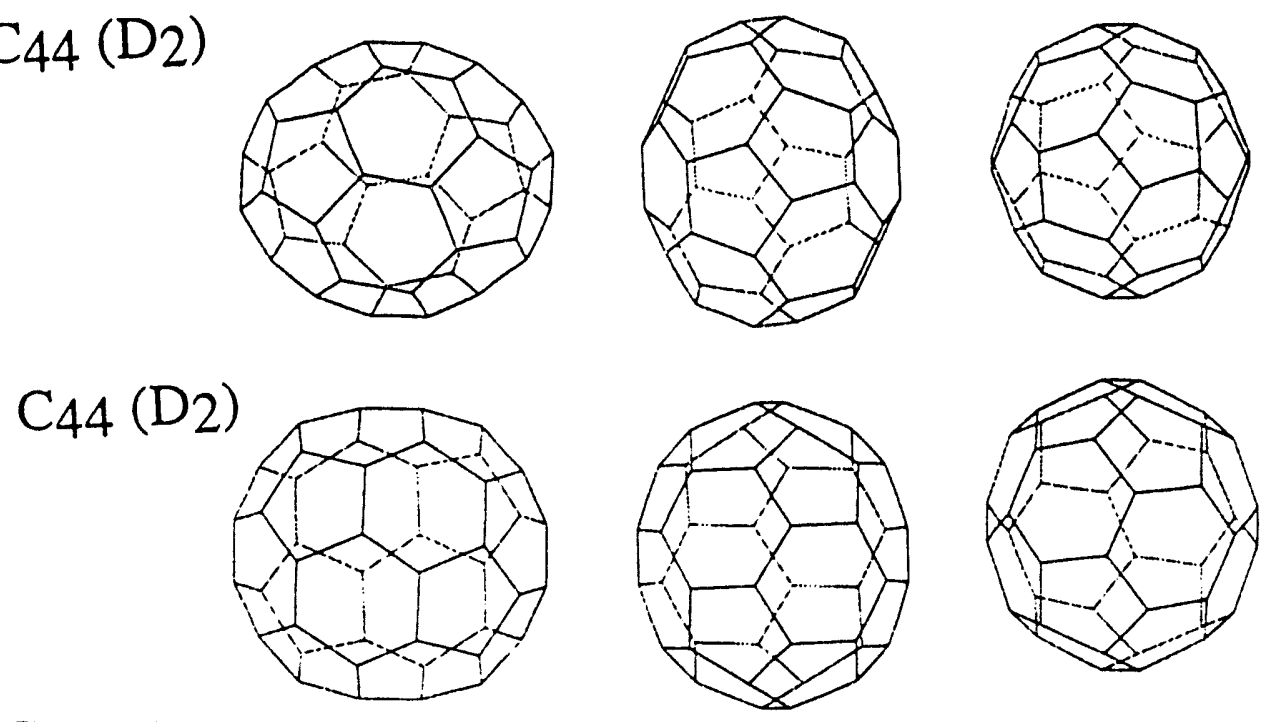

$\mathrm{C}_{46}\left(\mathrm{C}_{2}\right)$
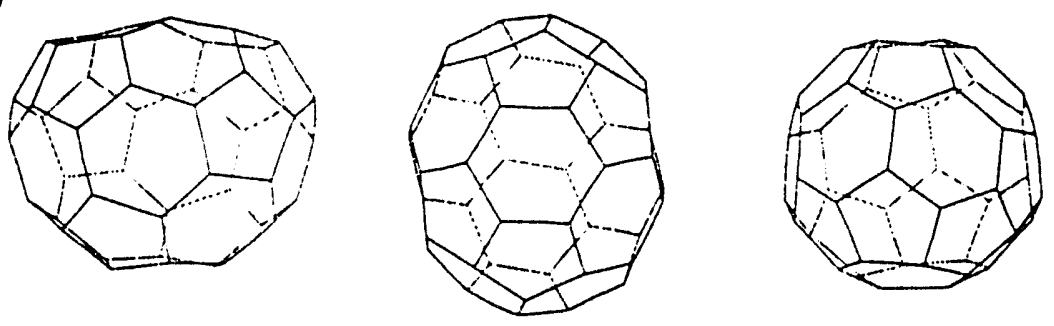

$\mathrm{C}_{48}\left(\mathrm{C}_{2}\right)$
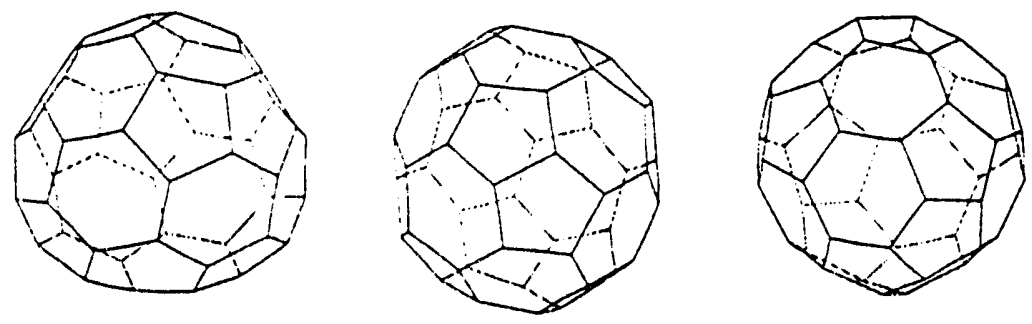

Figure 6.1 (Continued) 
C50 (D5h)
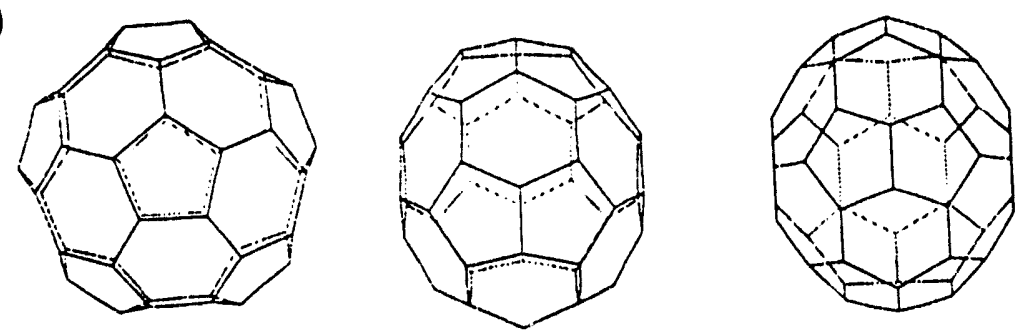

C50 (D3)
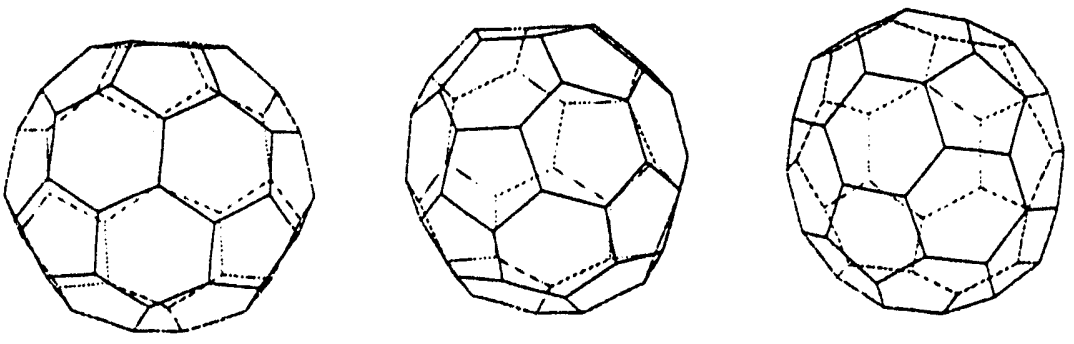

$\mathrm{C}_{52}\left(\mathrm{C}_{2}\right)$
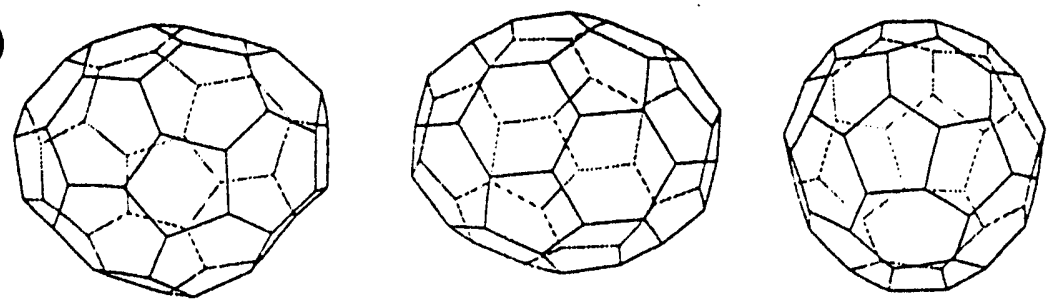

$\mathrm{C}_{54}\left(\mathrm{C}_{2 \mathrm{v}}\right)$
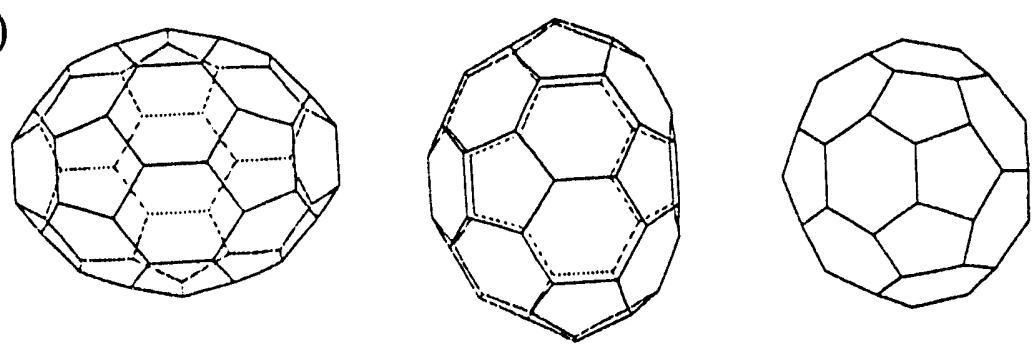

$\mathrm{C}_{56}(\mathrm{D} 2)$
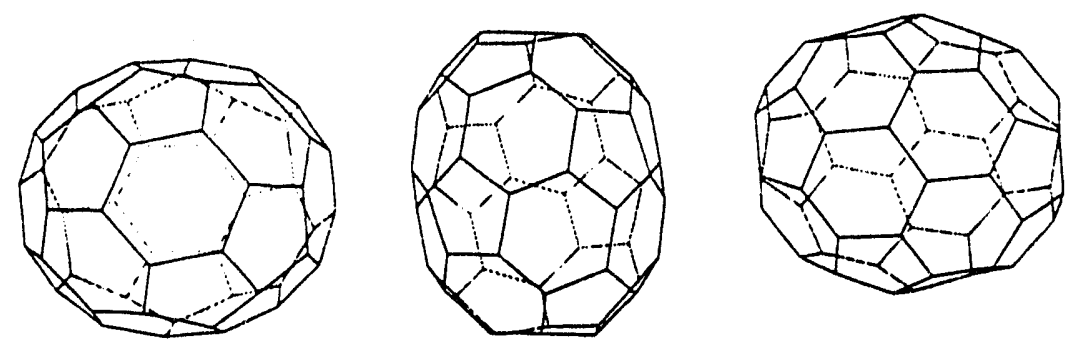

Figure 6.1 (Continued) 
$\mathrm{C}_{58}\left(\mathrm{C}_{\mathrm{S}}\right)$
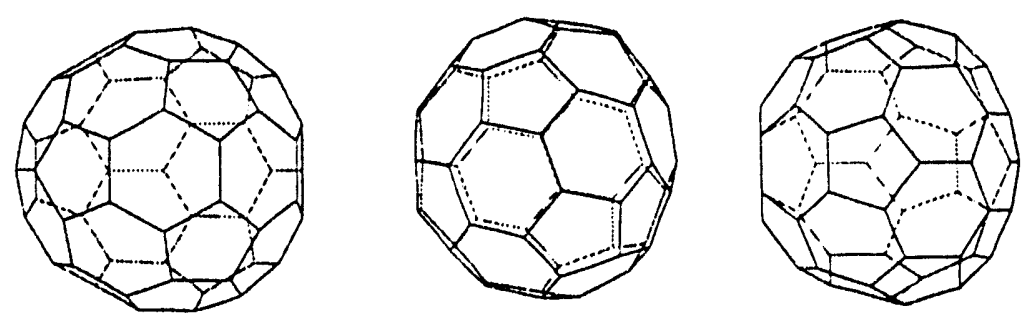

$\mathrm{C}_{60}\left(\mathrm{I}_{\mathrm{h}}\right)$
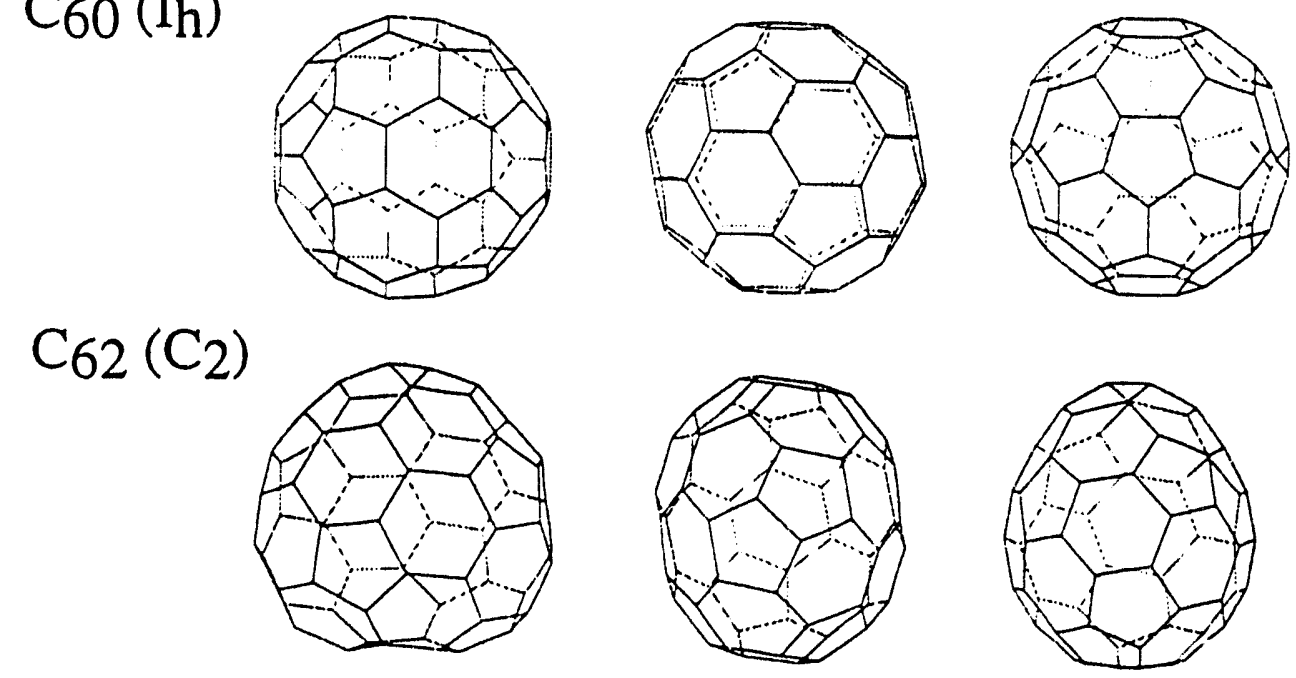

$\mathrm{C}_{64}\left(\mathrm{D}_{2}\right)$
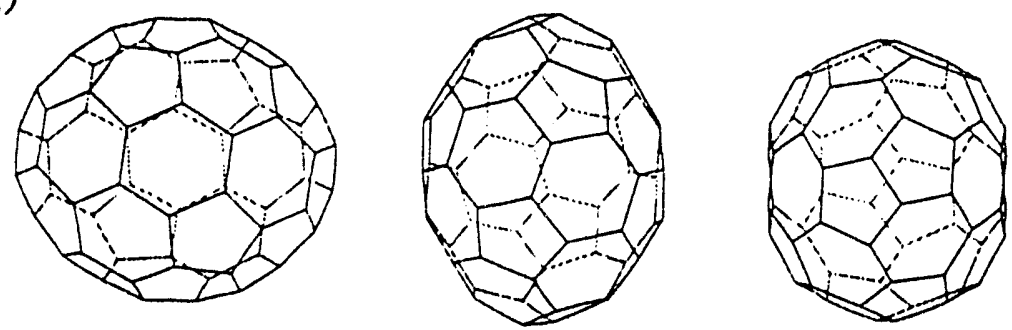

$\mathrm{C}_{66}\left(\mathrm{C}_{2}\right)$
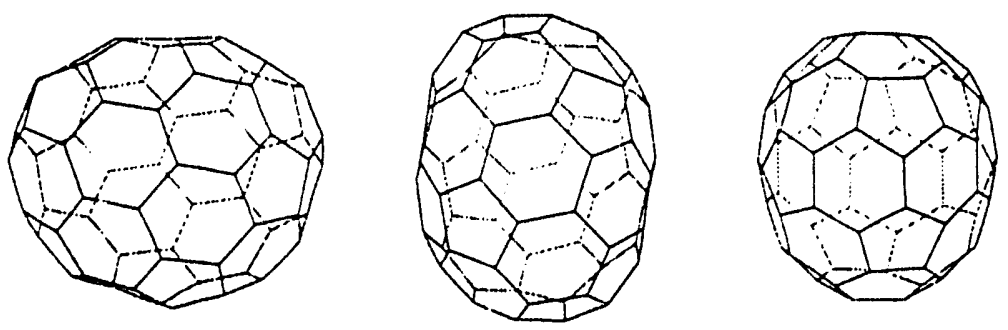

Figure 6.1 (Continued) 

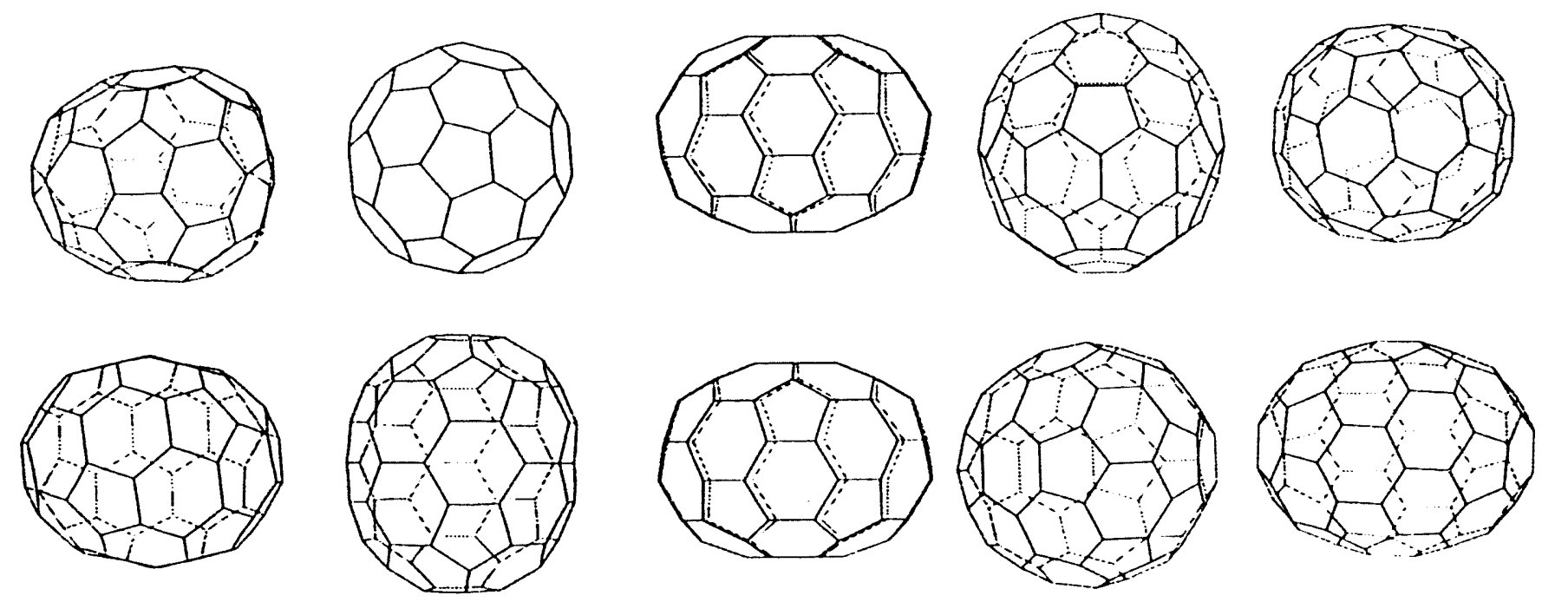

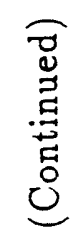
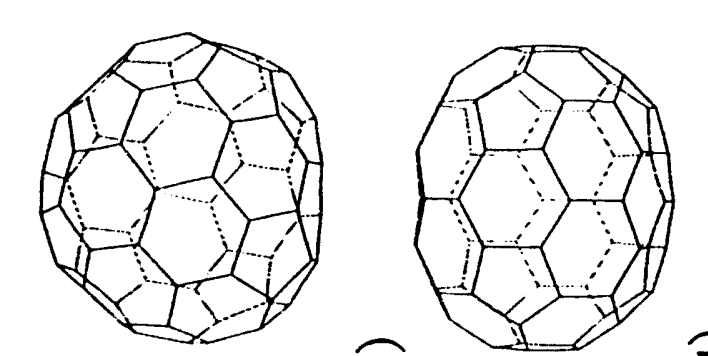

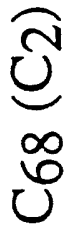

$\underbrace{2}_{0}$

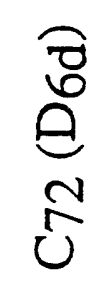
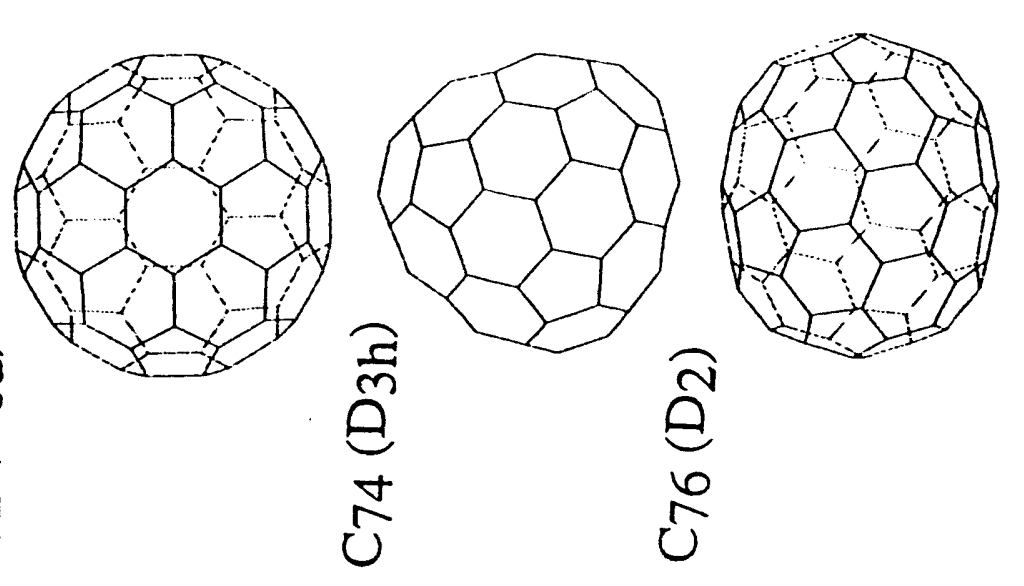

$-$

品

部 
$\mathrm{C}_{78}\left(\mathrm{C}_{2} \mathrm{v}^{\prime}\right)$

$\mathrm{C}_{78}\left(\mathrm{C}_{2 \mathrm{v}}\right)$
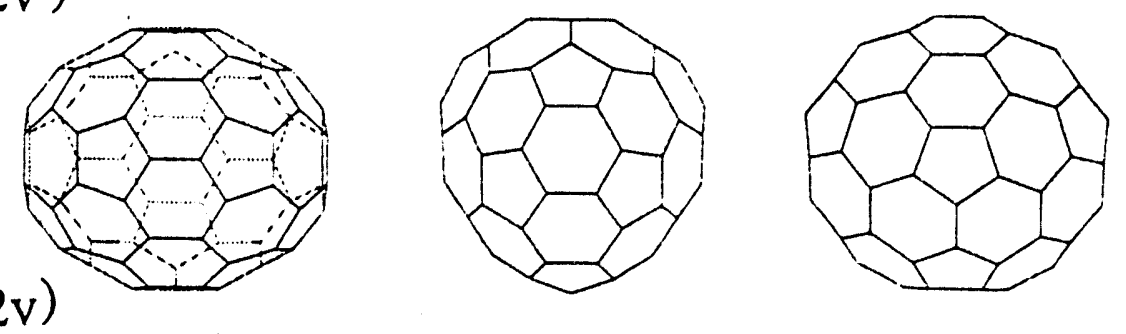

C78 (D3h')
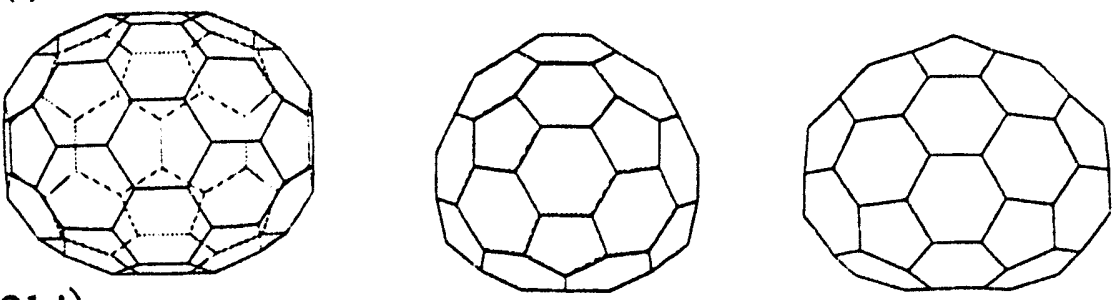

C78 (D3)
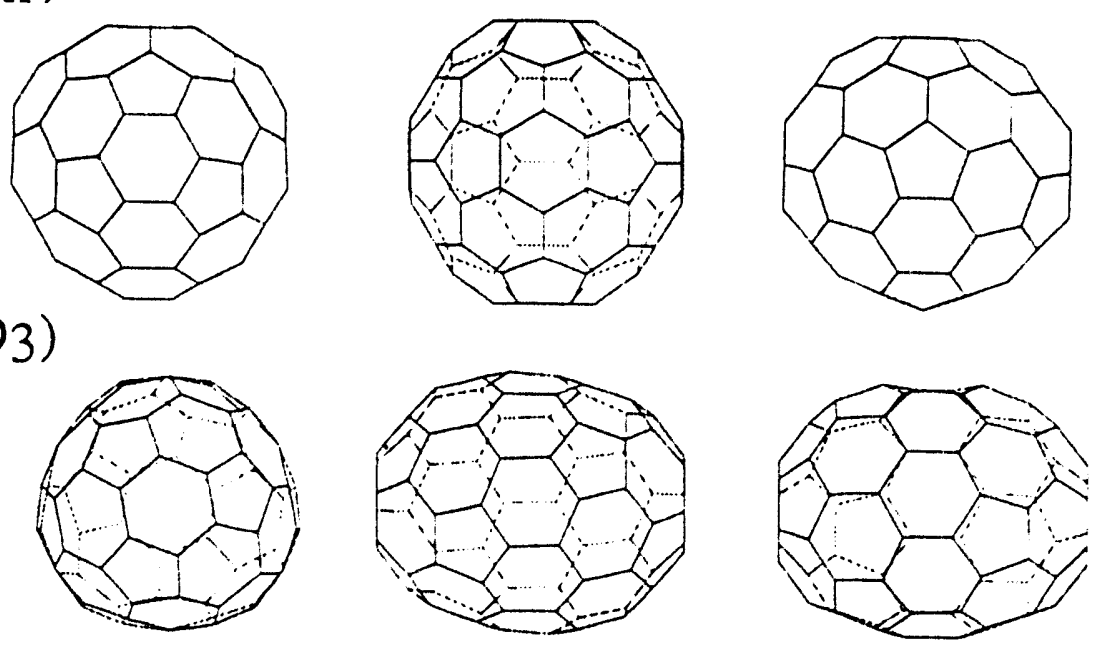

C78 (D3h)
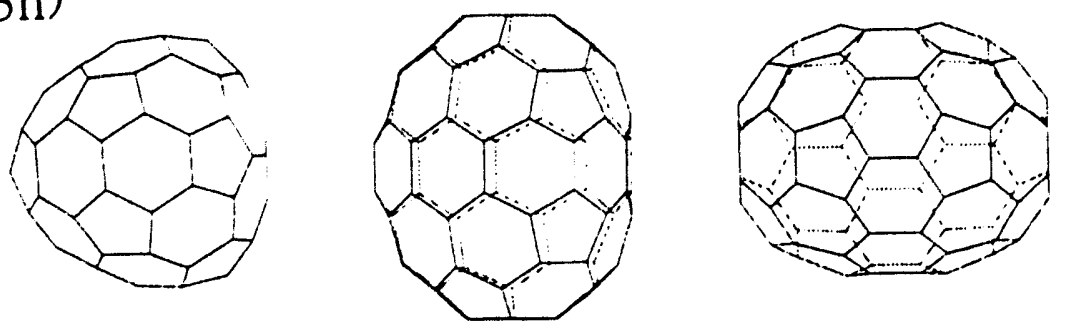

Figure 6.1 (Continued) 


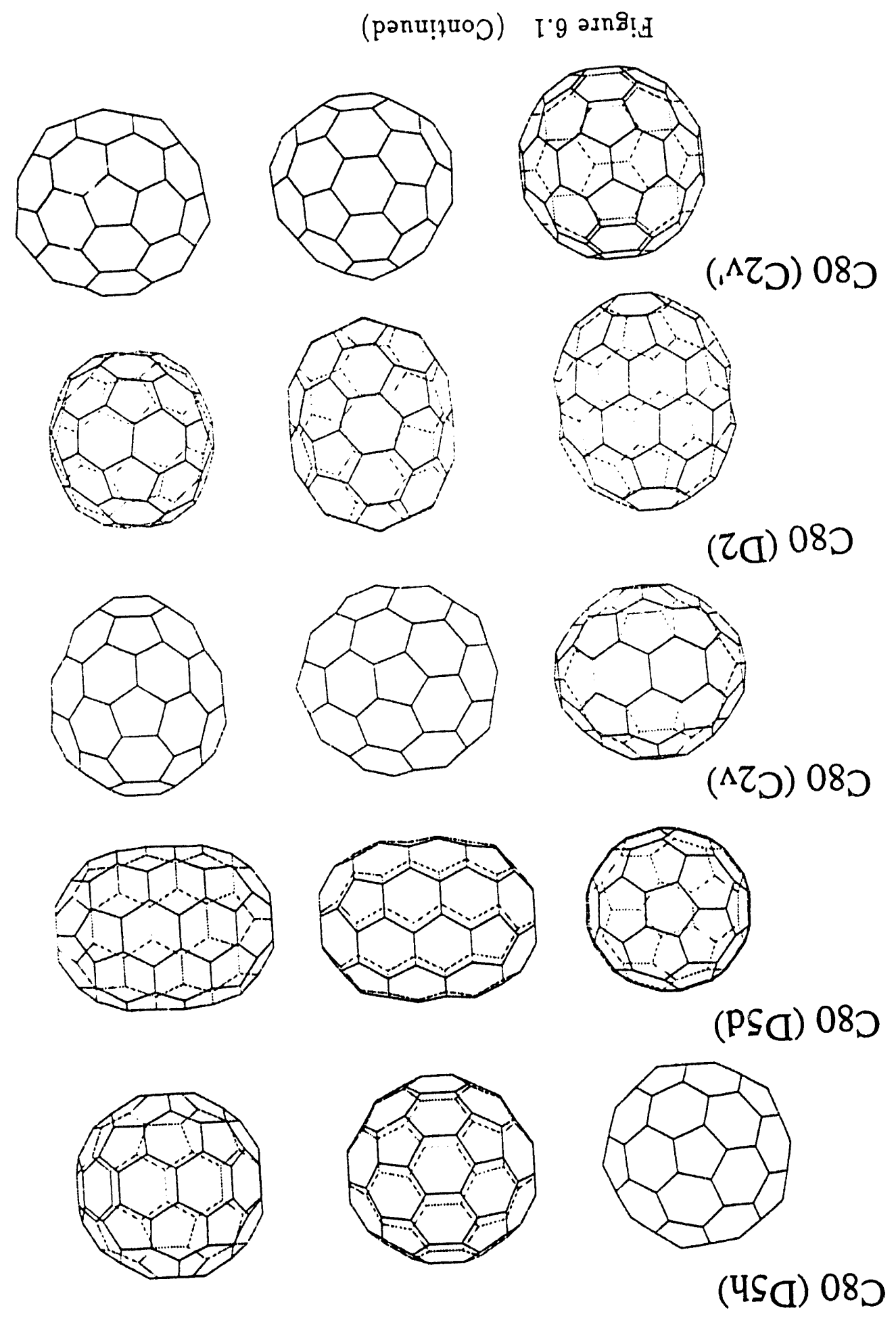




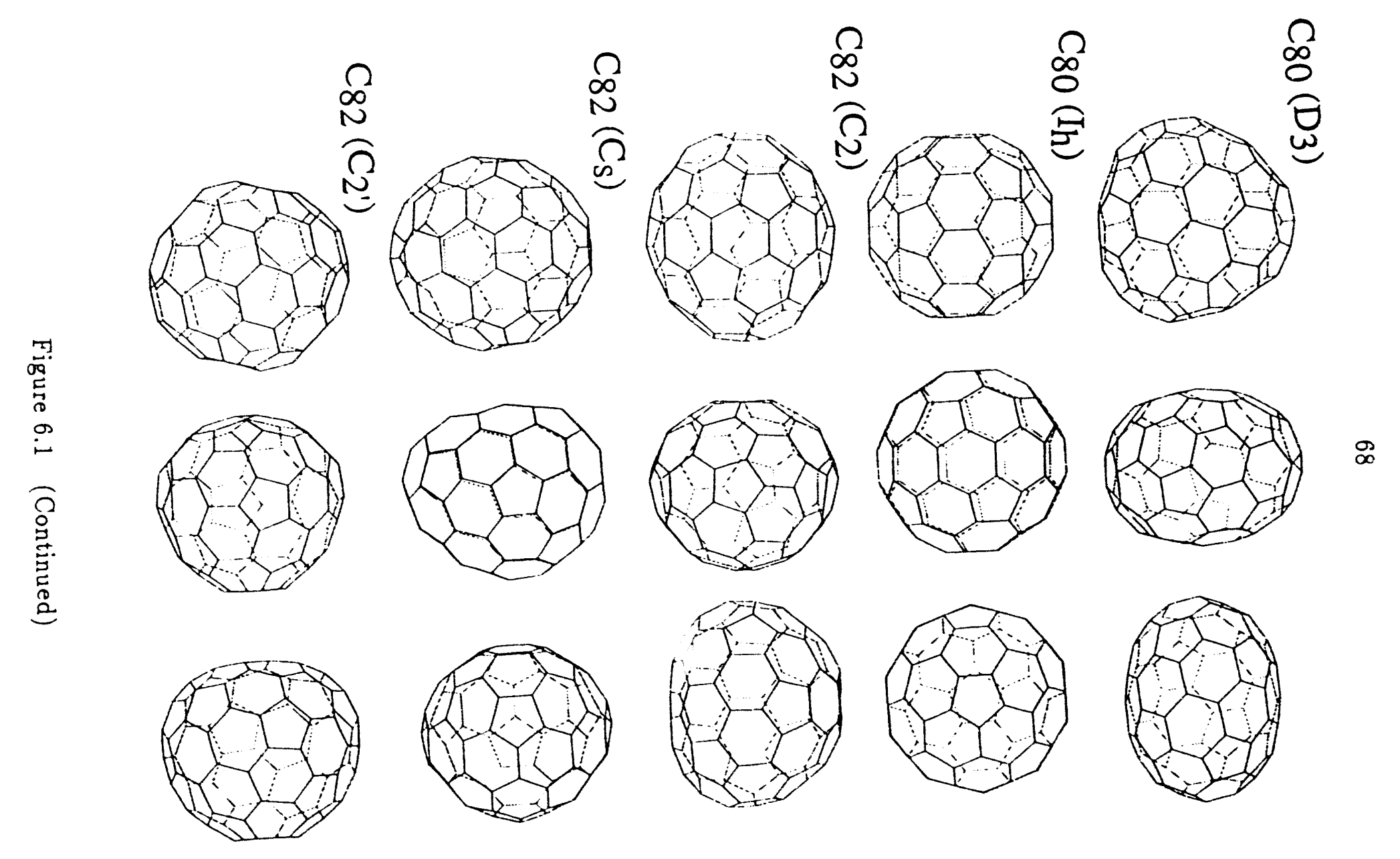


$\mathrm{C}_{82}\left(\mathrm{C}_{2} "\right)$

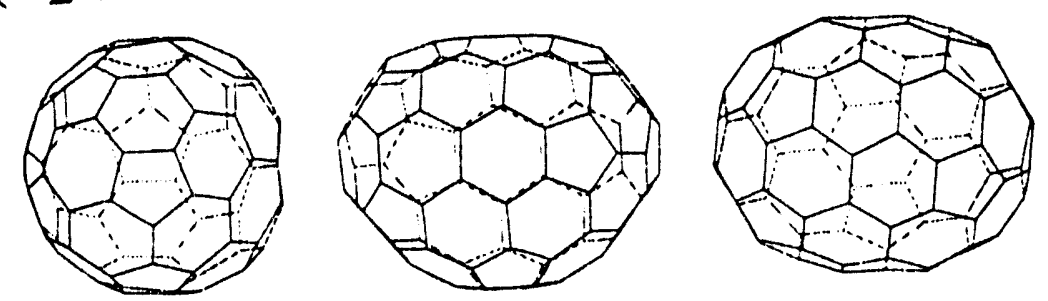

$\mathrm{C}_{82}\left(\mathrm{C}_{\mathrm{s}^{\prime}}\right)$
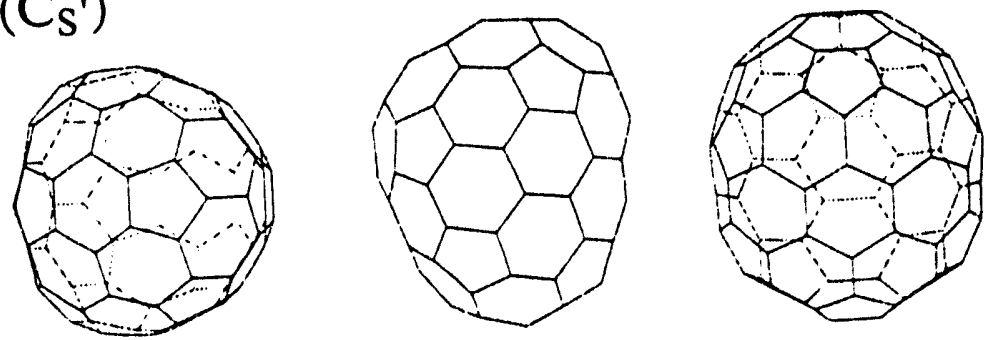

$\mathrm{C}_{82}$ ( $\mathrm{C}_{\mathrm{s}}$ ")
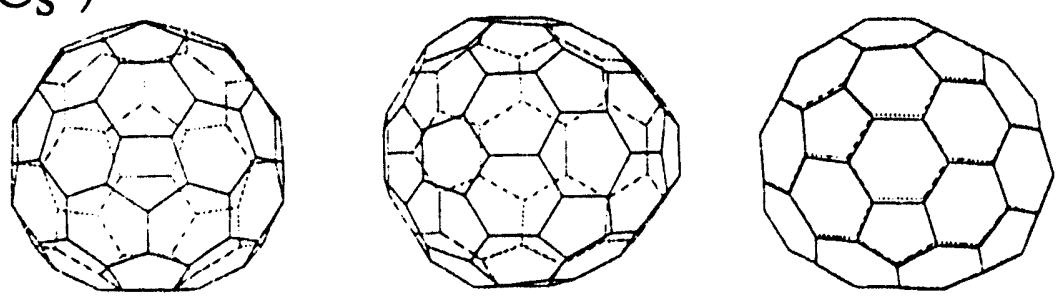

$\mathrm{C}_{82}\left(\mathrm{C}_{2 \mathrm{v}}\right)$
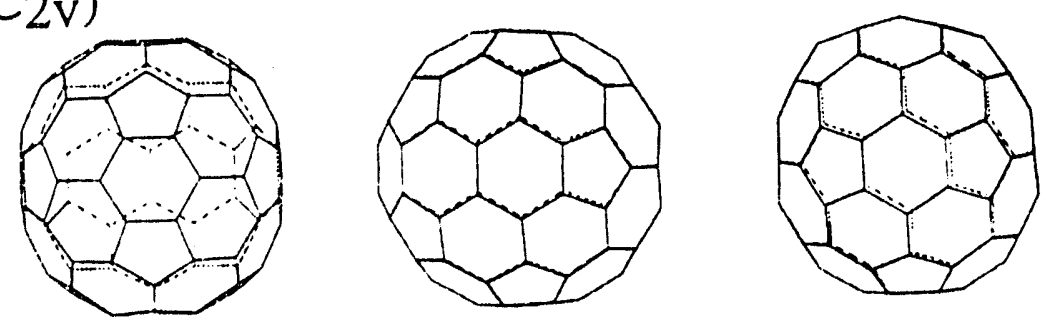

$\mathrm{C}_{82}$ (C3v)
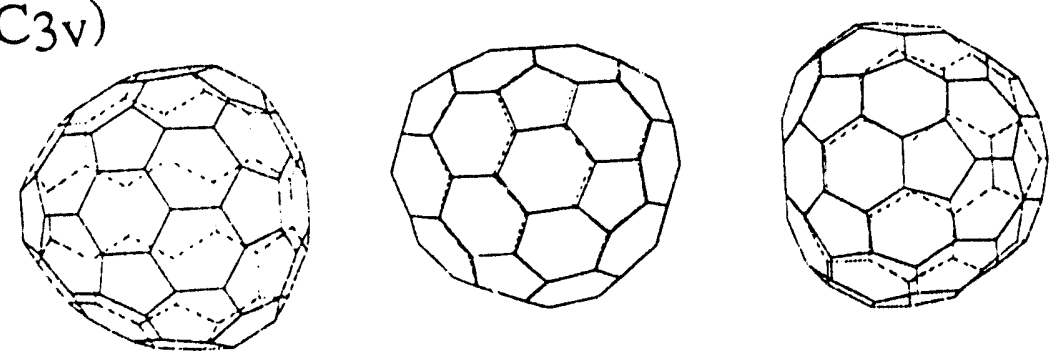

Figure 6.1 (Continued) 
$\mathrm{C} 82\left(\mathrm{C}_{3} \mathrm{v}^{\prime}\right)$
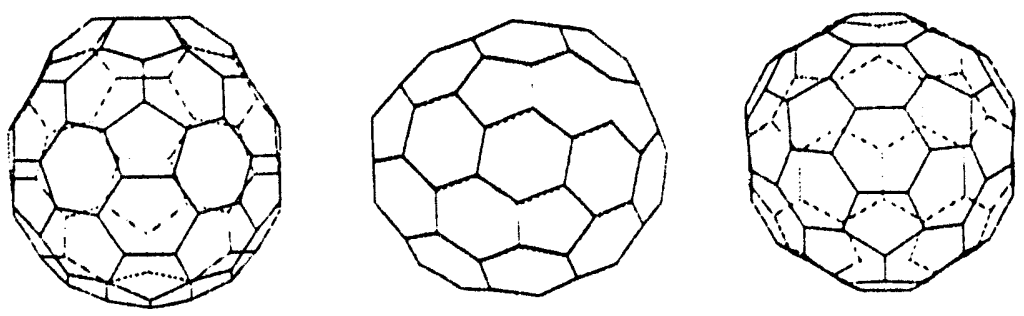

$\mathrm{C} 84$ (D2)22
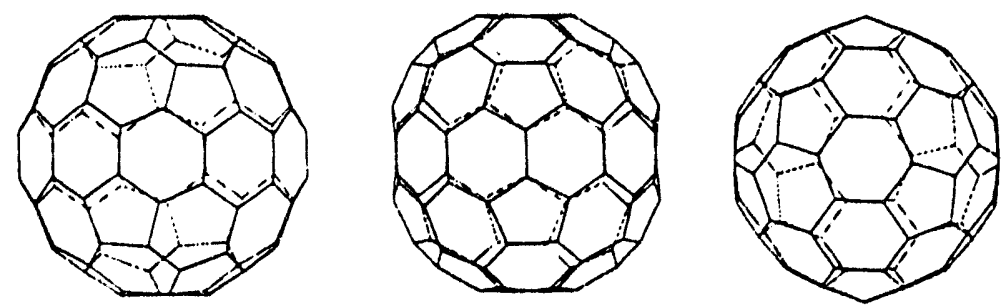

C84 (D2d)23
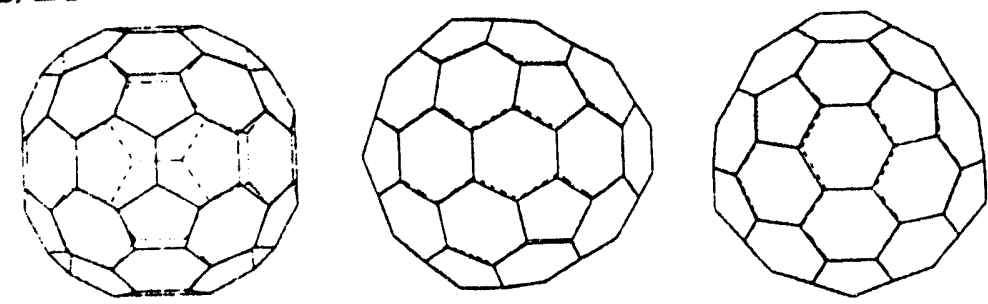

$\mathrm{C}_{86}\left(\mathrm{C}_{1}\right)$
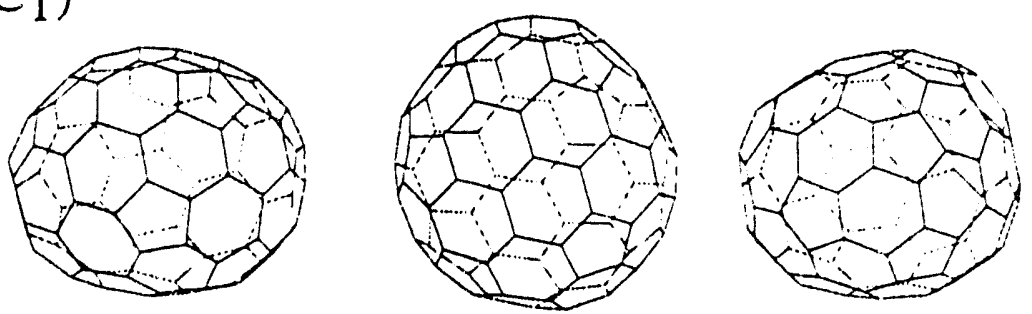

$\mathrm{C}_{86}\left(\mathrm{C}_{2}\right)$
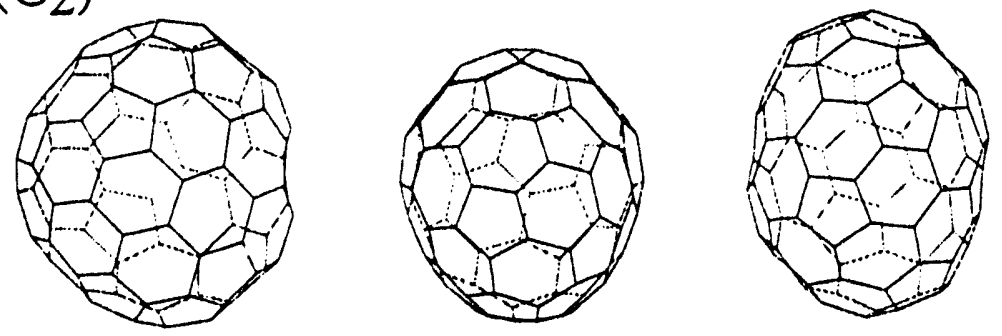

Figure 6.1 (Continued) 
$\mathrm{C}_{88}\left(\mathrm{C}_{2}\right)$

$\mathrm{C}_{90}\left(\mathrm{C}_{2}\right)$
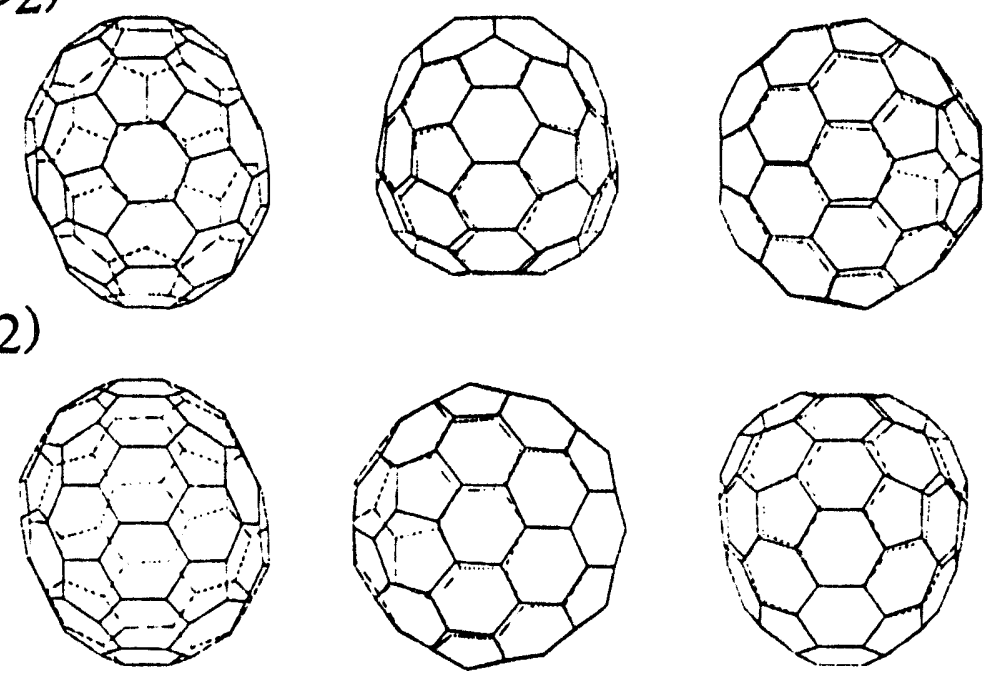

$\mathrm{C}_{90}\left(\mathrm{C}_{2 \mathrm{v}}\right)$
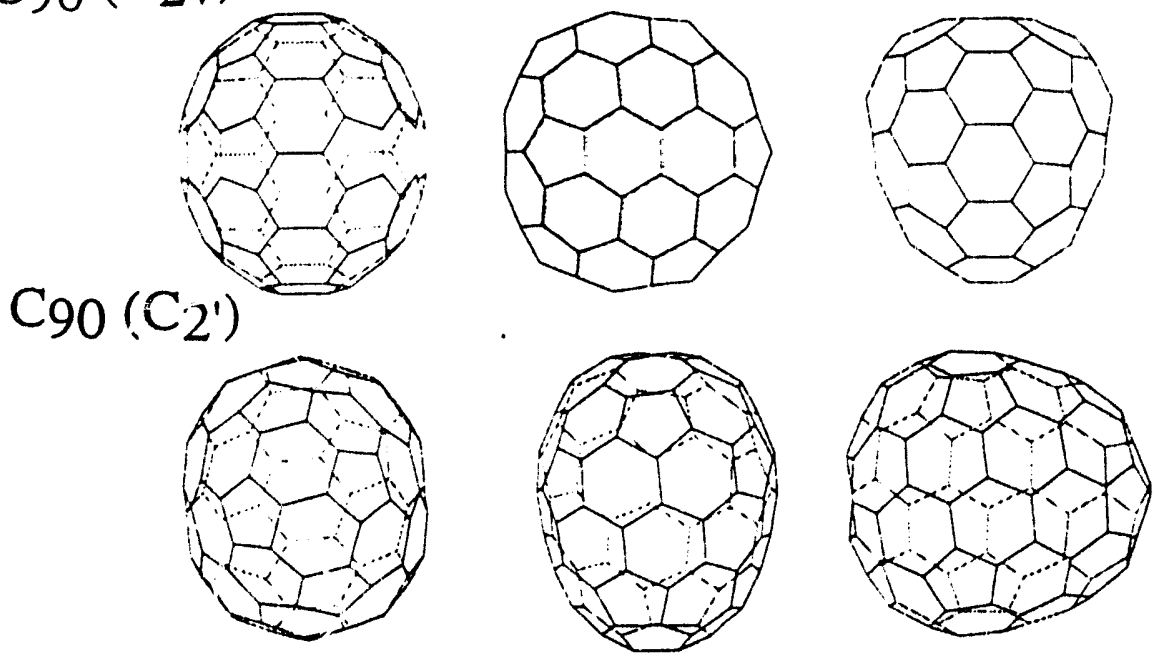

$\mathrm{C}_{92}\left(\mathrm{C}_{2}\right)$
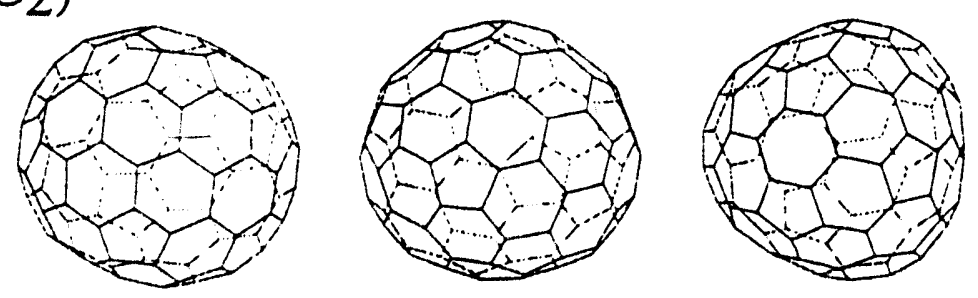

Figure 6.1 (Continued) 


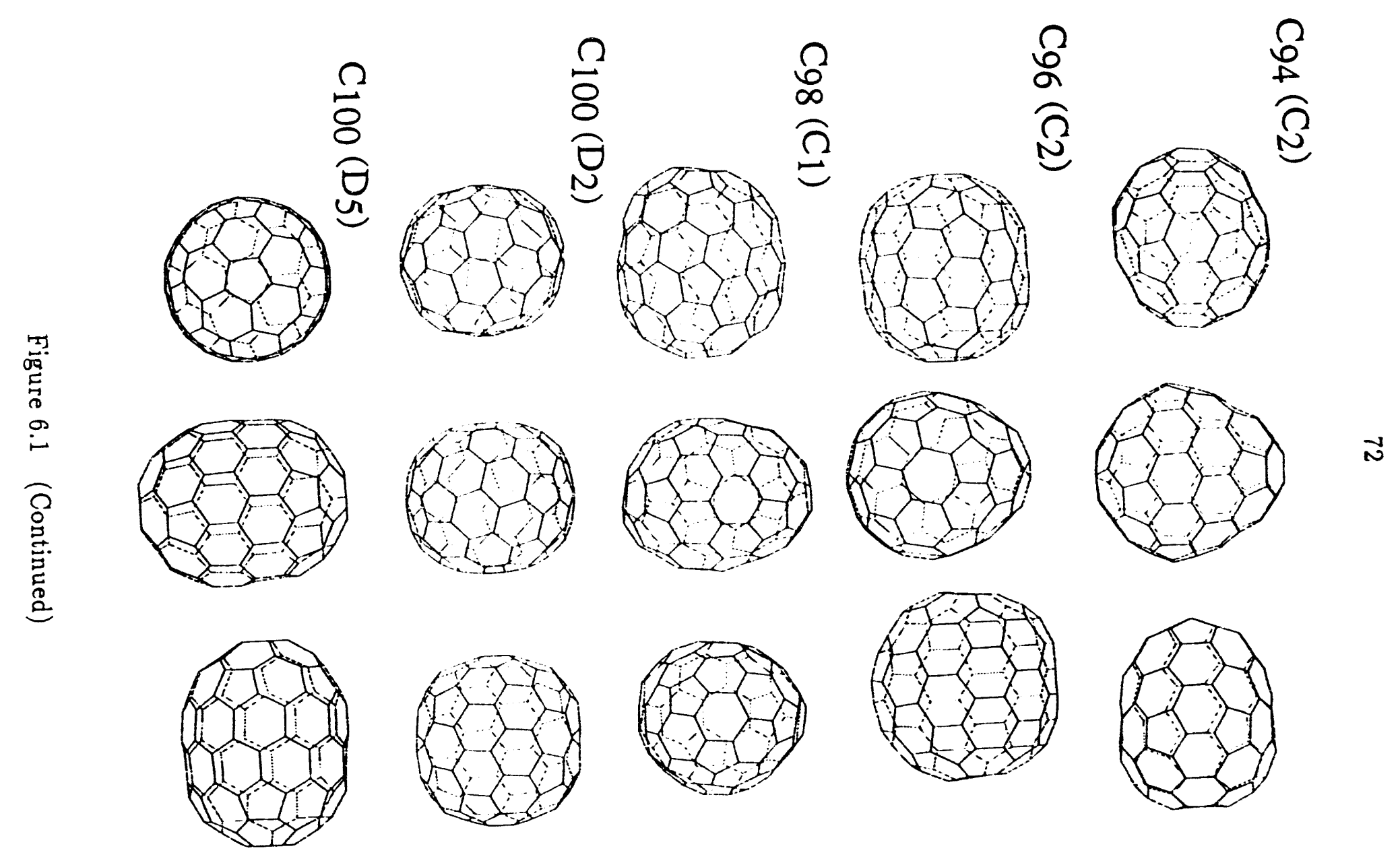


(1) Isolated-pentagon rule:

One of the most important empirical rules previously proposed for the construction of carbon fullerenes is the so called isolated-pentagon rule. To demonstrate this rule, we chose $\mathrm{C}_{60}$ as an example and estimate how much energy it costs to bring a pair of pentagons next to each other. By rotating a single $\mathrm{C}_{2}$-unit by 90 degrees, we can interchange the positions of a pair of pentagons and hexagons [103] (see Fig. 6.4). Such a rearrangement brings two pairs of pentagons adjacent to each other in the $\mathrm{C}_{60}$ network (topologically there is no way to form a $\mathrm{C}_{60}$ network with a single pair of touching pentagons). The binding energy of the $\mathrm{C}_{60}$ molecule is found to decrease by $1.4 \mathrm{eV}(0.7 \mathrm{eV}$ per pair), which is quite a large amount of energy. For large fullerenes, this rule is very useful to narrow down the possible candidates enormously. For example, there are only 5 isolated-pentagon isomers out of 11229 possible isomers for $\mathrm{C}_{78}[97]$. However, we found that not every isolated-pentagon isomer is more stable than all the touching-pentagon isomers. In the case of $\mathrm{C}_{84}$, the most unstable $\mathrm{D}_{2}(1)$ isomer is $1.9 \mathrm{eV}$ per molecule smaller in the binding energy than that of the ground-state $\mathrm{D}_{2}(22)$ isomer and it is topologically possible for $\mathrm{C}_{84}$ to form isomers with a single pair of touching pentagons. Since a pair of touching pentagons only costs $0.7 \mathrm{eV}$, it is very likely that $\mathrm{C}_{84}$ can have a touching-pentagon isomer which is more stable than the $\mathrm{D}_{2}(1)$ isomer. Later, we indeed found one touching-pentagons isomer which is $0.34 \mathrm{eV}$ per molecule more stable than the $D_{2}(1)$ isomer. For larger fullerenes, to have several pentagons closer can be compensated for by having a larger (flatter) hexagon-only region and it is anticipated that this kind of trade will be more beneficial as the clusters become larger. Still, within the range of molecules considered in this study, the ground-state isomers we obtained are 
all isolated-pentagon isomers if these fullerenes have an isolated-pentagon isomer.

It is also interesting to note that all the fullerenes in the range from $\mathrm{C}_{20}$ to $\mathrm{C}_{70}$, except $\mathrm{C}_{60}$ and $\mathrm{C}_{70}$, do not have isolated-pentagon isomers. Then in this regime, the isolated-pentagon rule needed to be generalized to that isomers favor the geometries which separate the pentagons as far apart as possible. One good example to demonstrate this rule is $\mathrm{C}_{50}$ (see Fig. 6.1) which is interesting to many people due to its relative abundance under gentle, one photon $F_{2}$ excimer-laser ionization of $\mathrm{C}_{60}$. The ground-state isomer was first proposed by Kroto [106] which has $\mathrm{D}_{5 h}$ symmetry. In a later paper, Manolopoulos [96] suggested that an isomer with $D_{3}$ symmetry should be more stable than the $\mathrm{D}_{5 h}$ isomer by using Huckel theory. From our calculations, we found that the ground-state isomer is the $\mathrm{D}_{5 h}$ isomer which is more stable than the $\mathrm{D}_{3}$ isomer by about $0.23 \mathrm{eV}$ per molecule. This is in accord with our generalized isolated-pentagon rule since the $D_{3}$ isomer has six pairs of touching pentagons while the $\mathrm{D}_{5 h}$ isomer has only five pairs.

(2) Symmetry:

All the structures obtained by our scheme in this range consist of six- and fivemembered rings only. Unlike $\mathrm{C}_{60}$ and $\mathrm{C}_{70}$, most of the ground-state fullerene structures have very low symmetries and a few of them have no symmetry at all (see Table 6.2). In general, high symmetry restricts the distribution of pentagons. This point is well demonstrated by the $D_{2}$ and $D_{5}$ isomer of $C_{100}$ (see Fig. 6.1). In the $D_{5}$ isomer of $\mathrm{C}_{100}$, the 12 pentagon are confined to the two ends of a sausage-like cage in order to satisfy the $D_{5}$ symmetry, while in the $D_{2}$ isomer, the 12 pentagons are spread much more uniformly throughout the cage surface. As a result, the energy of the $\mathrm{D}_{5}$ isomer is $0.6 \mathrm{eV}$ per molecule higher than that of the $\mathrm{D}_{2}$ isomer. 
(3) Mixture of isomers:

From the above analysis, we found that the lowest excited isomer for $C$ stable by $1.4 \mathrm{eV}$ per molecule compared to the ground-state isomer. '. : difference is quite large so that only the ground-state $\mathrm{I}_{h}$ isomer of $\mathrm{C}_{60}$ is $u \ldots \ldots$ experimentally. However, our study shows that some clusters have several isomers which are very close in energy. For example, there is another $D_{2}$ isomer of $C_{44}$ besides the ground-state $\mathrm{D}_{2}$ structure. The energy difference of these two isomers is only $0.037 \mathrm{eV}$ per molecule and they also have almost the same HOMO-LUMO energy separation. It can be expected that these isomers will coexist in a mixture if one day $\mathrm{C}_{44}$ can be isolated. In fact, the phenomenon of coexistence of different isomers for a given fullerene size has already been observed in recent experiments $[43,45]$ for larger fullerenes, e.g. $\mathrm{C}_{78}, \mathrm{C}_{82}$ and $\mathrm{C}_{84}$.

(4) Jahn-Teller Distortion:

Jahn-Teller distortions [107] may occur for lullerenes in which the highest occupied molecular orbit (HOMO) is degenerate and partially occupied. For the fullerenes we have studied, there are quite a number of structures with Jahn-Teller distortions (see Table 6.2). Although the symmetry of the topological network can be as high as $\mathrm{I}_{h}$ (in $\mathrm{C}_{20}$ ), the real symmetry is reduced to $\mathrm{C}_{2}$ after the geometry is fully optimized.

In the following, we discuss several important fullerenes which are both experimentally and theoretically interesting.

(1) $C_{76}:$

Using the ring-spiral algorithm [96], Manolopoulos determined that there are two isolated-pentagon $\mathrm{C}_{76}$ isomers [98]. Based on the size of HOMO-LUMO gaps, he proposed a chiral structure for $\mathrm{C}_{76}$ which was subsequently confirmed by experi- 
ment [42]. Both isolated-pentagon isomers were generated from our scheme and we found that the chiral $\mathrm{D}_{2}$ isomer is more stable than the $\mathrm{T}_{d}$ isomer by $0.24 \mathrm{eV}$ per molecule.

$$
\text { (2) } C_{78}:
$$

This fullerene has five distinct isomers satisfying the isolated-pentagon rule [97]. We found that the $\mathrm{C}_{2 v^{\prime}}$ is the ground-state structure and the energies of $\mathrm{D}_{3 h^{\prime}}, \mathrm{C}_{2 v}, \mathrm{D}_{3}$ and $\mathrm{D}_{3 h}$ are $0.09,0.28,0.32$, and $0.91 \mathrm{eV}$ per molecule, respectively, higher than that of the ground-state $\mathrm{C}_{2}$ ' isomer. Later, the structure of $\mathrm{C}_{78}$ has been analysed by the NMR technique by two independent groups with different conclusions. Diederich et al. [43] suggested that the product of $\mathrm{C}_{78}$ is a mixture of $\mathrm{C}_{2 v}$ and $\mathrm{D}_{3}$ isomers with a ratio of $5: 1$, while Kikuchi et al. [45] concluded that the mixture consists of $\mathrm{C}_{2 v^{\prime}}$, $\mathrm{C}_{2 v}$ and $\mathrm{D}_{3}$ isomers in the ratio of $5: 2: 2$. The second result is in agreement with our calculation that $\mathrm{C}_{2 v^{\prime}}$ isomer is the most stable one for $\mathrm{C}_{78}$. However, we should note that both experiments did not find the $\mathrm{D}_{3 h^{\prime}}$ isomer which is also energetically favoured in our calculations and the first-principles calculations [110]. This may be due to the fact that the $\mathrm{D}_{3 h^{\prime}}$ isomer has a relatively small HOMO-LUMO energy gap which makes it more reactive than other isomers. Both the absence of $\mathrm{D}_{3 h^{\prime}}$ isomer and the different results from the two different experiments suggest that kinetic factors may play an important role in the selection of products.

(3) $C_{82}$ :

The energy diagram containing all 9 isolated-pentagon isomers of $\mathrm{C}_{82}$ is plotted in Fig. 6.2 and the most stable isomer has $\mathrm{C}_{2}$ symmetry. This agrees with the experimental observation [45] that there are 41 strong NMR peaks. In addition, our calculation pinpoints the specific $\mathrm{C}_{2}$ isomer among three possible $\mathrm{C}_{2}$ isomers satisfy- 
ing the isolated-pentagon rule. Beside the larger binding energy, the ground-state $\mathrm{C}_{2}$ isomer has a substantially bigger HOMO-LUMO gap which is very important in the isolation process. (This point will be discussed more in the next section). However a detailed analysis of other weak NMR peaks leads to $\mathrm{C}_{2 v}$ and $\mathrm{C}_{3 v}$ isomers and they are not favoured in our calculations in both binding energies and HOMO-LUMO gaps. The chemical shifts measured by NMR are decided by site environment and distinct atoms not related by symmetry presumably have different chemical shifts. But for large fullerenes, the environment of atoms at different isomers can be very close and consequently the chemical shifts are almost identical. So it is not trivial to assign these weak peaks with so many different isomers and unknown relative abundance ratio. Only the measurement of separated isomers will definitely characterize the minor isomers in the $\mathrm{C}_{82}$ sample. The most interesting property of this fullerene is that it is the magic number for metal-encapsulating fullerenes $\mathrm{MC}_{82}$ [37]. (where $\mathrm{M}$ are $\mathrm{Zr}, \mathrm{Ti}, \mathrm{Ca})$ Since $\mathrm{C}_{82}$ has several isomers very competitive with each other, the geometric structure of the carbon cage in ground-state $\mathrm{C}_{82}$ and $\mathrm{MC}_{82}$ are not necessary the same. Further study is required to examine how the donated charge from the metal atom changes the relative stabilities and the electronic properties of the fullerenes.

(4) $C_{84}:$

Besides $\mathrm{C}_{60}$ and $\mathrm{C}_{70}, \mathrm{C}_{84}$ is the most abundant fullerene observed in the mass spectra [41]. The energy diagram of all 24 isolated-pentagon isomers is plotted in Fig. 6.3 and the $\mathrm{D}_{2}(22)$ and $\mathrm{D}_{2 d}(23)$ isomers are energetically the most favourable structures. The ball and stick model of $\mathrm{D}_{2 d}$ isomer and four $\mathrm{D}_{2}$ isomers are plotted in Fig. 6.4. (The notation follows the work of Manopolous [100]) This result is 


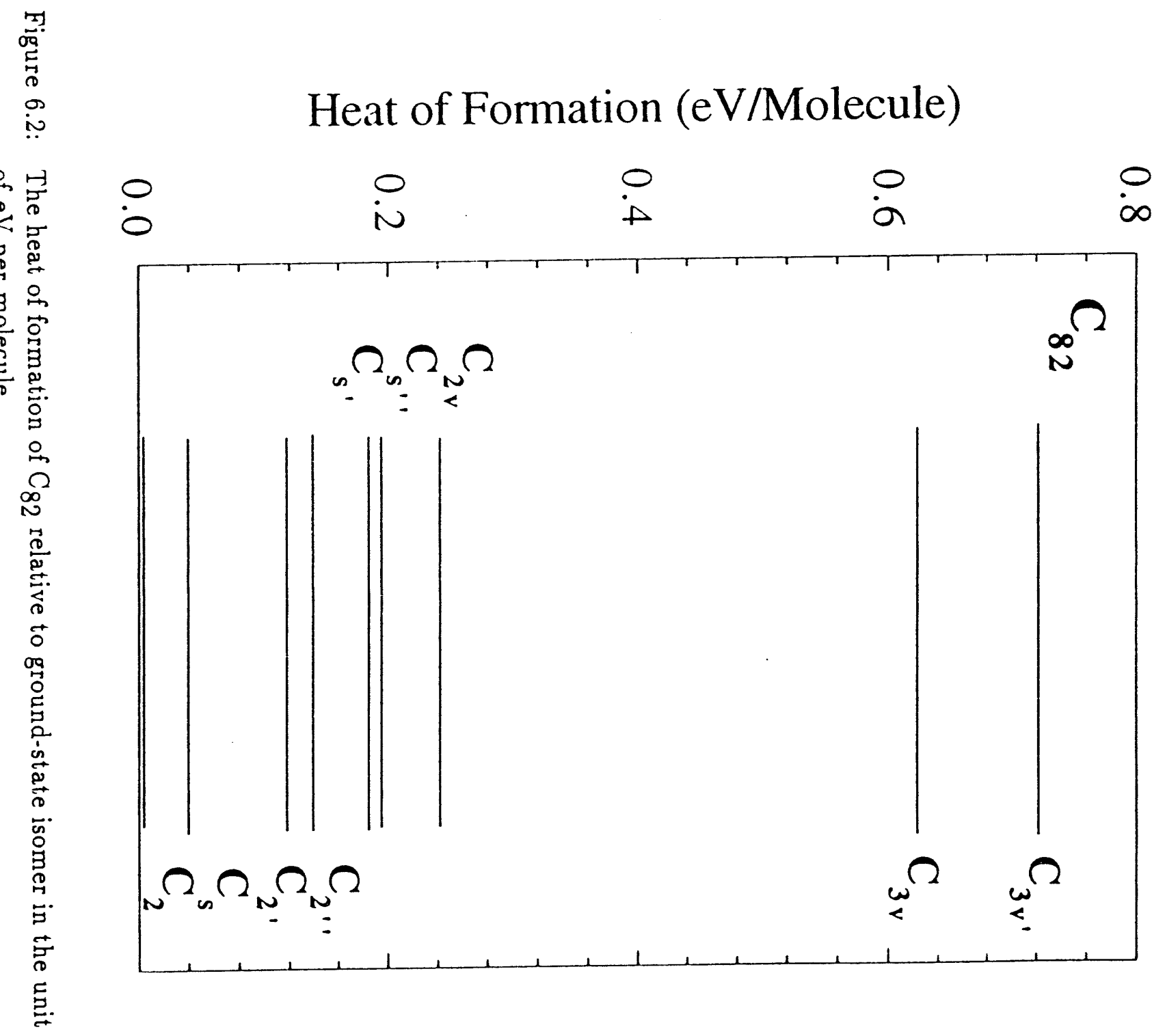


supported by first-principles calculations $[108,111]$. Later, Kikuchi et al. $[45]$ had characterized the structures of the $\mathrm{C}_{84}$ isomers by NMR measurements and they did find a mixture of $\mathrm{D}_{2}$ and $\mathrm{D}_{2 d}$ isomers with a $2: 1$ ratio. However, a ring-stacking model proposed by Wakabayashi and Achiba et al. [109] favours the $\mathrm{D}_{2}(5)$ isomer. Although, the number of NMR peaks of these two isomers is the same, the spectra of photoemission are more close to the electronic density states calculated for the $\mathrm{D}_{2}(22)$ isomer [112].

From Fig. 6.4, we can see that the shape of these two $D_{2}$ isomers are quite different: while the $\mathrm{D}_{2}(22)$ isomer is spherical like the $\mathrm{D}_{2 d}$ isomer, the $\mathrm{D}_{2}(5)$ is relatively elongated. So the diffusion speeds of $\mathrm{D}_{2}(22)$ and $\mathrm{D}_{2}(5)$ in the chromatograph should be quite different. In the experiments, people [113] found that it is very difficult to separate the $\mathrm{D}_{2}$ and $\mathrm{D}_{2 d}$ isomers in the chromatograph. This implies that the shape of $\mathrm{D}_{2}$ is quite close to spherical $\mathrm{D}_{2 d}$ isomer and indirectly supports the existence of $\mathrm{D}_{2}(22)$ isomer in the sample.

(5) $C_{90}, C_{94}$ and $C_{96}$ :

These high fullerenes are also relatively abundant in the mass spectra but they have not been isolated yet. The numbers of possible isolated-pentagon isomers for $\mathrm{C}_{90}$ and $\mathrm{C}_{94}$ are already quite numerous: 46 and 134 respectively [100]. Instead of generating and checking each structure one by one, based on the successes in the previous cases, we think we can get the lowest energy isomers from our limited search. The number of isomers tested for $\mathrm{C}_{90}, \mathrm{C}_{94}$ and $\mathrm{C}_{96}$ in this calculation are 10,12 and 18 respectively. The energetically favoured isomers are drawn in the Fig. 6.1. and they all have relatively low symmetry. This information can be very helpful for analysing the complicated experimental data. 


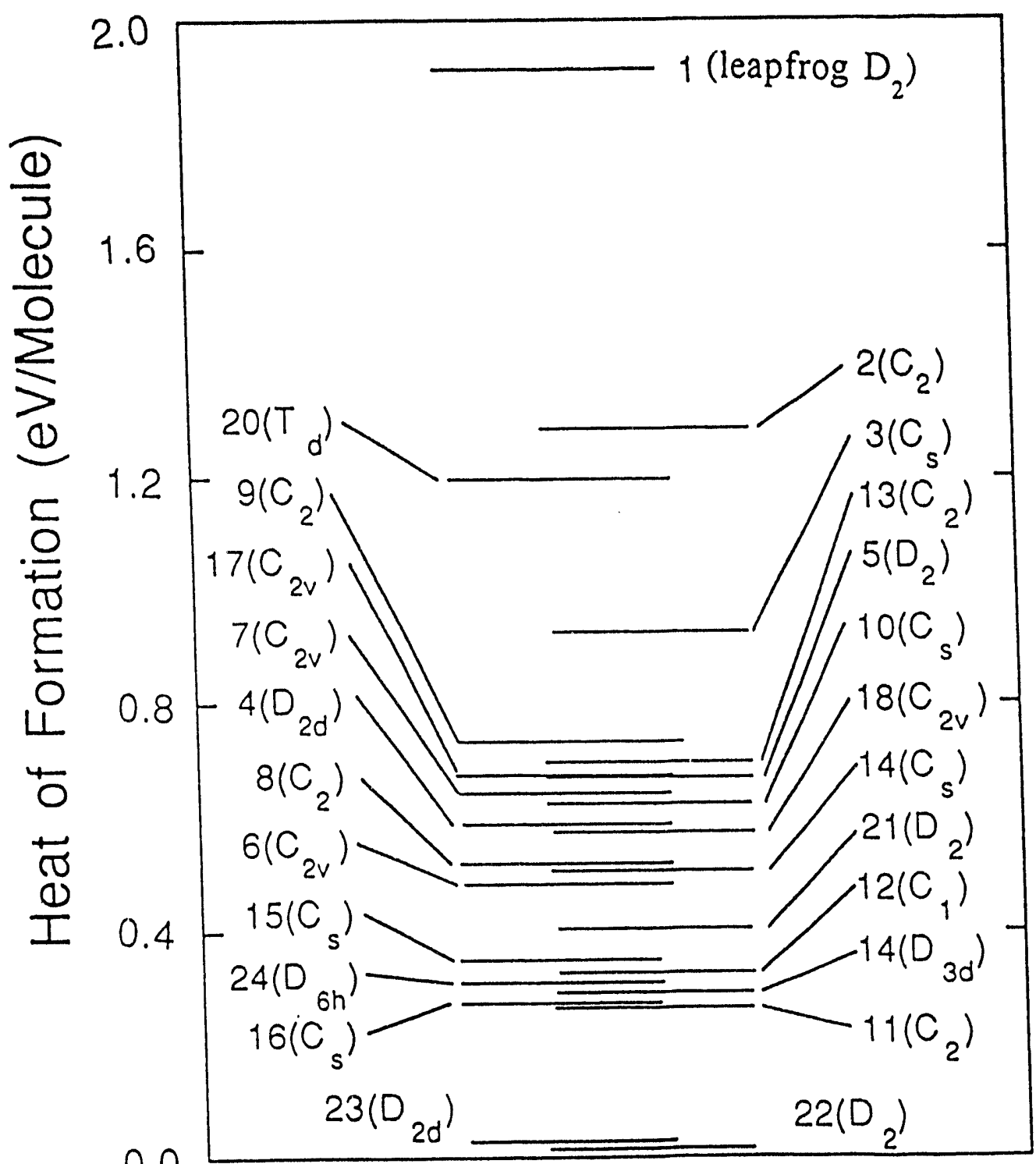

Figure 6.3: The heats of formation of $\mathrm{C}_{84}$ isomers relative to ground-state isomer $22\left(\mathrm{D}_{2}\right)$ in units of $\mathrm{eV} /$ molecule. 


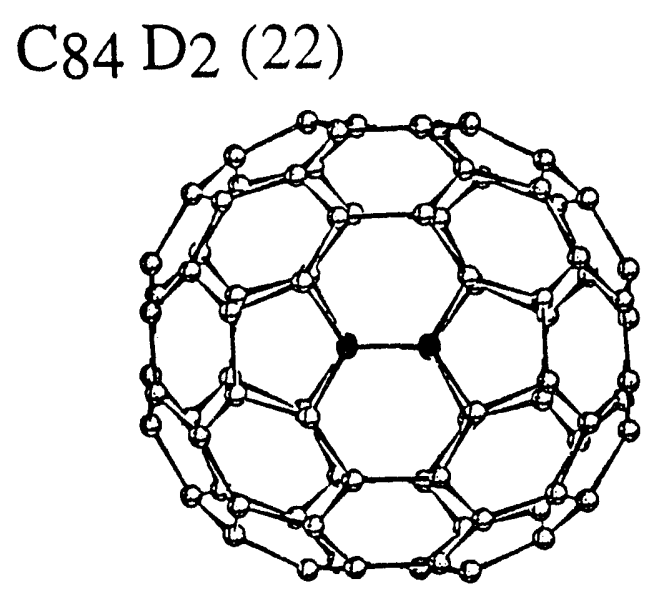

C84 D2d (23)

C84 D2 (21)
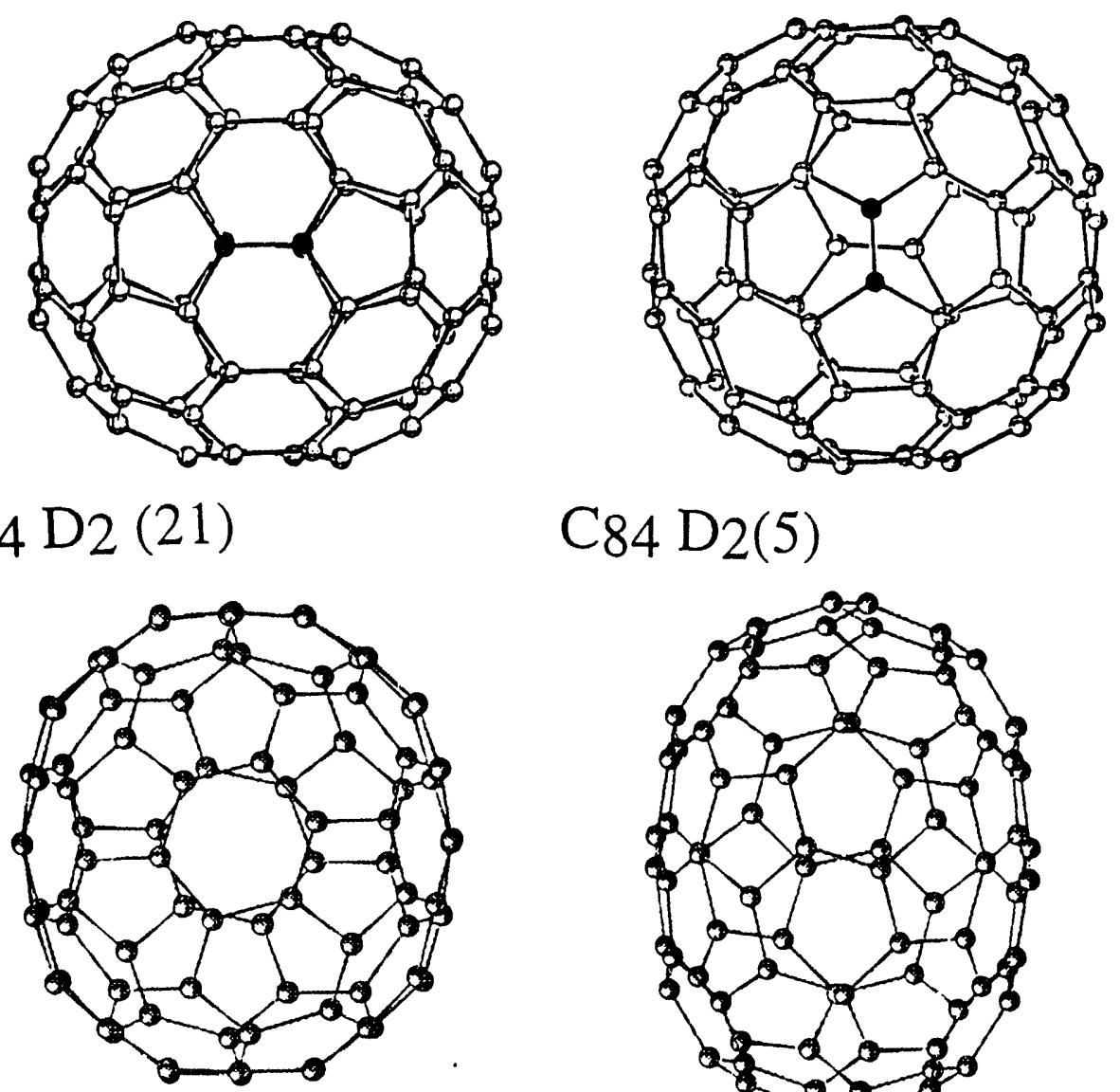

C84 D2(5)

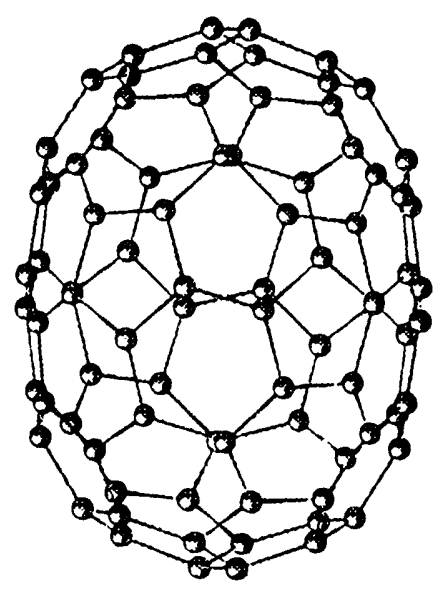

C84 D2 (1)

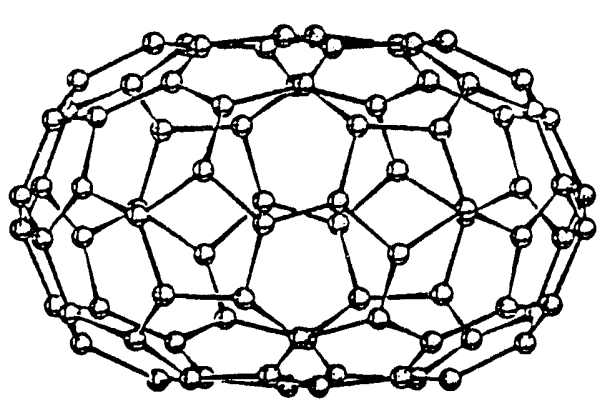

Figure 6.4: Ball and stick models of $D_{2 d}$ and four $D_{2}$ isomers. 


\section{Stabilities of Fullerenes}

The heats of formation of the ground-state fullerenes as a function of cluster size are plotted in Fig. 6.5. We found that the formation energies of small cages grow very rapidly as the cluster size is increased. This behaviour can be related to the rapidly decreasing number of adjacent pentagons as the cluster size increases. On the other hand, the energies of large fullerenes, which have no adjacent pentagons, are found to increase at a much slower rate. The most interesting feature is that $\mathrm{C}_{60}$, $\mathrm{C}_{70}$ and $\mathrm{C}_{84}$, which are the magic numbers observed in the experiments, are found to be more stable than their neighbors.

Although the extraordinary abundance of $\mathrm{C}_{60}$ and $\mathrm{C}_{70}$ has been known for several years, the underlying origin of this phenomenon is still not well understood. Recently, other minor abundance peaks have also been observed for $\mathrm{C}_{76}, \mathrm{C}_{78}, \mathrm{C}_{84}$, $\mathrm{C}_{90}$, and $\mathrm{C}_{96}$, but not for every even-numbered clusters in this range. It seems a complete explanation of the experimental observation has to involve kinetic factors in addition to thermodynamic stability. Otherwise all fullerenes larger than $\mathrm{C}_{70}$ would be more abundant than $\mathrm{C}_{60}$ according to theis heats of formation. From our present systematic study, we found that the abundance peaks of the mass spectra can be correlated with the fragmentation stabilities and the electronic HOMO-LUMO gaps of the fullerenes.

Since the exact fragmentation process of carbon fullerenes is still unclear, we limit our consideration to a process with successive $\mathrm{C}_{2}$ loss as suggested by O'Brien et al. $[114,115]$. The fragmentation energy in this process is defined as:

$$
E_{f r a g}(N)=E_{c o h}(N)-E_{c o h}(N-2)-E_{c o h}(2) .
$$




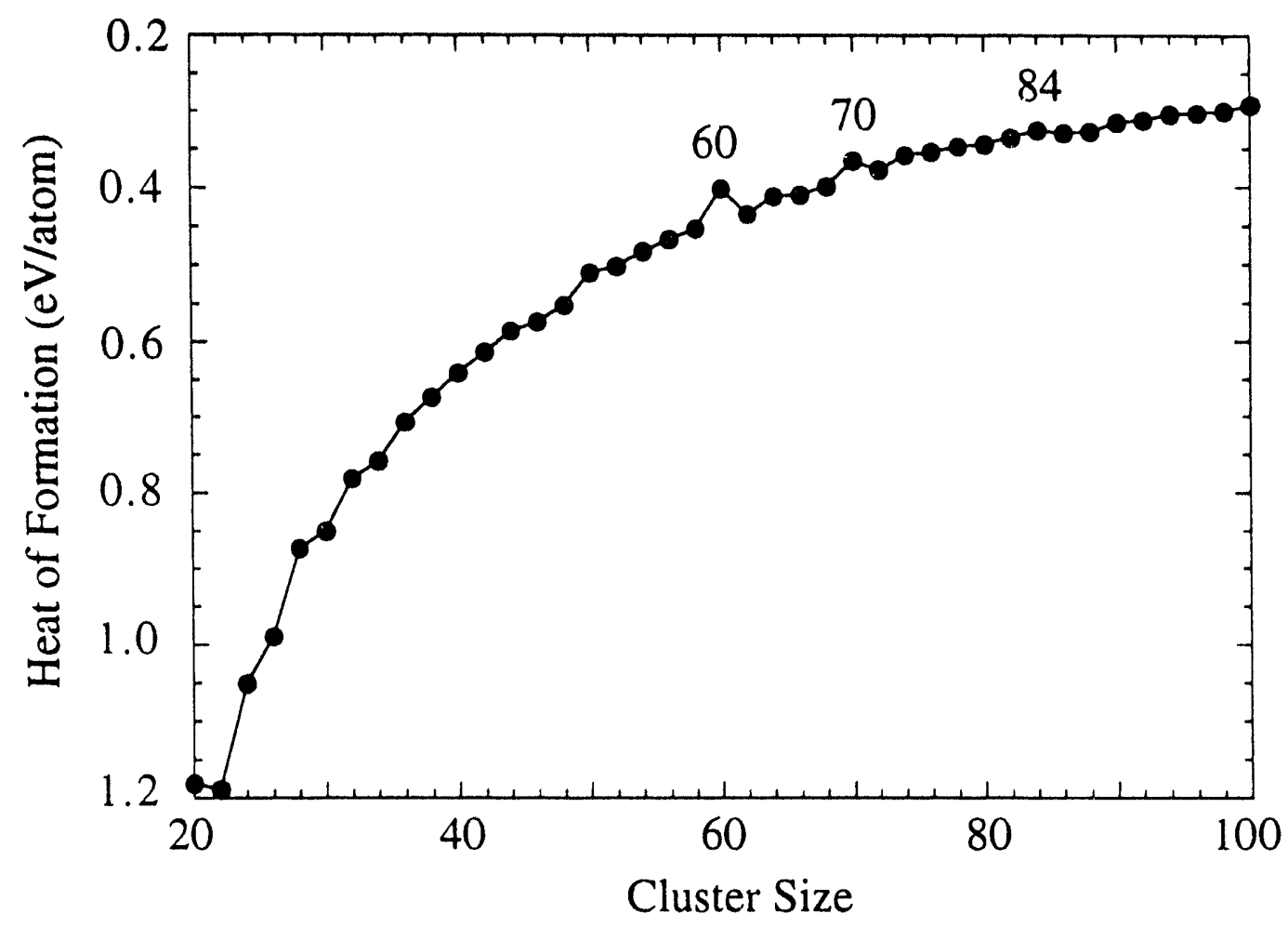

Figure 6.5: The heat of formation of carbon clusters relative to bulk graphite as a function of cluster size $\mathrm{N}$. 
here $\mathrm{E}_{\text {coh }}(\mathrm{N})$ is the cohesive energy of a $\mathrm{C}_{N}$ cluster and $\mathrm{E}_{f r a g}(\mathrm{~N})$ is the energy needed for $C_{N}$ to fragment into $C_{2}$ and $C_{N-2}$. Therefore clusters with larger fragmentation energy will be more stable against fragmentation. In Fig. 6.6(a), the fragmentation energy as a function of cluster size obtained from our study is plotted. It is clearly shown that $\mathrm{C}_{60}$ and $\mathrm{C}_{70}$ have large fragmentation energies with respect to their neighboring fullerenes. This may explain why $\mathrm{C}_{60}$ and $\mathrm{C}_{70}$ are superstable. However it is not clear from the fragmentation energy why $\mathrm{C}_{74}, \mathrm{C}_{80}, \mathrm{C}_{86}$ and $\mathrm{C}_{88}$ do not show abundance peaks comparable with $\mathrm{C}_{84}$.

In the work of Manolopoulos et al. [96], they suggested using the HOMO-LUMO energy separation as criterion for the kinetic stability of fullerenes. While it is still not clear to us how the HOMO-LUMO gap can control fullerene formation at high temperature, we believe that it is the key parameter controlling the chemical reactivity of the fullerene during the extraction and isolation process. A big HOMO-LUMO energy separation makes it more difficult to extract electrons from the low-lying HOMO or add electrons to the high-lying LUMO. So it is easy for the clusters with a small HOMO-LUMO energy gap to react with the solvent and other encountered chemicals. A bigger HOMO-LUMO gap might also make the cluster more stable towards further accretion of extra $\mathrm{C}$ atoms. In Fig. 6.6(b), our results for the HOMO-LUMO gap are plotted as a function of cluster size. It is clearly shown that the $\mathrm{C}_{60}$ and $\mathrm{C}_{70}$ not only have very large fragmentation energies, but also have very large HOMO-LUMO gaps. These results are strongly correlated to the extraordinary abundance of these two fullerenes. It is interesting to see that $\mathrm{C}_{74}, \mathrm{C}_{80}, \mathrm{C}_{86}$ and $\mathrm{C}_{88}$, which are absent in the mass spectra, all have very small HOMO-LUMO gaps. Due to the chemical reactivity discussed above, these fullerene should be less stable during the isolation 
process.

Finally, we would like to point it that an explanation of experimental mass spectra based solely on the consideration of HOMO-LUMO energy separation would not be satisfactory. An obvious example is $\mathrm{C}_{72}$ which has a very large HOMO-LUMO energy separation but it is not seen in mass spectra. Because from the fragmentation energy, $\mathrm{C}_{72}$ strongly favors losing a dimer to become $\mathrm{C}_{70}$ and it may not exist in the early formation stage. Our results shows that in order to explain the mass spectra of larger fullerenes, it is necessary to consider both the HOMO-LUMO gaps and the fragmentation energies simultaneously. The HOMO-LUMO gap measure the stability of a cluster against further chemical reaction, while the fragmentation energy measures the stability of the cluster against further fragmentation. The only exception in our calculation is $\mathrm{C}_{64}$. It has quite high peaks in both stabilities, but it is not observed in experiments. We suspect that $\mathrm{C}_{64}$ is too close to the superstable $\mathrm{C}_{60}$ and it may be unstable towards a fragmentation with a loss of $\mathrm{C}_{4}$.

\section{Summary}

The energetically most favorable fullerene structures have been determined by an efficient and accurate cage network generation scheme for all even-numbered carbon clusters from $\mathrm{C}_{20}$ to $\mathrm{C}_{100}$. We found that the governing rule for the selection of energetically favorable cage structures is the generalized isolated-pentagon rule in which the ground-state isomer tend to separate the pentagons as far apart as possible. The isomers with large HOMO-LUMO gaps will be more stable against chemical reaction and consequently will be easier to isolate. For most of the fullerenes, the ground-state isomers prefer low symmetry structures and usually there are several 


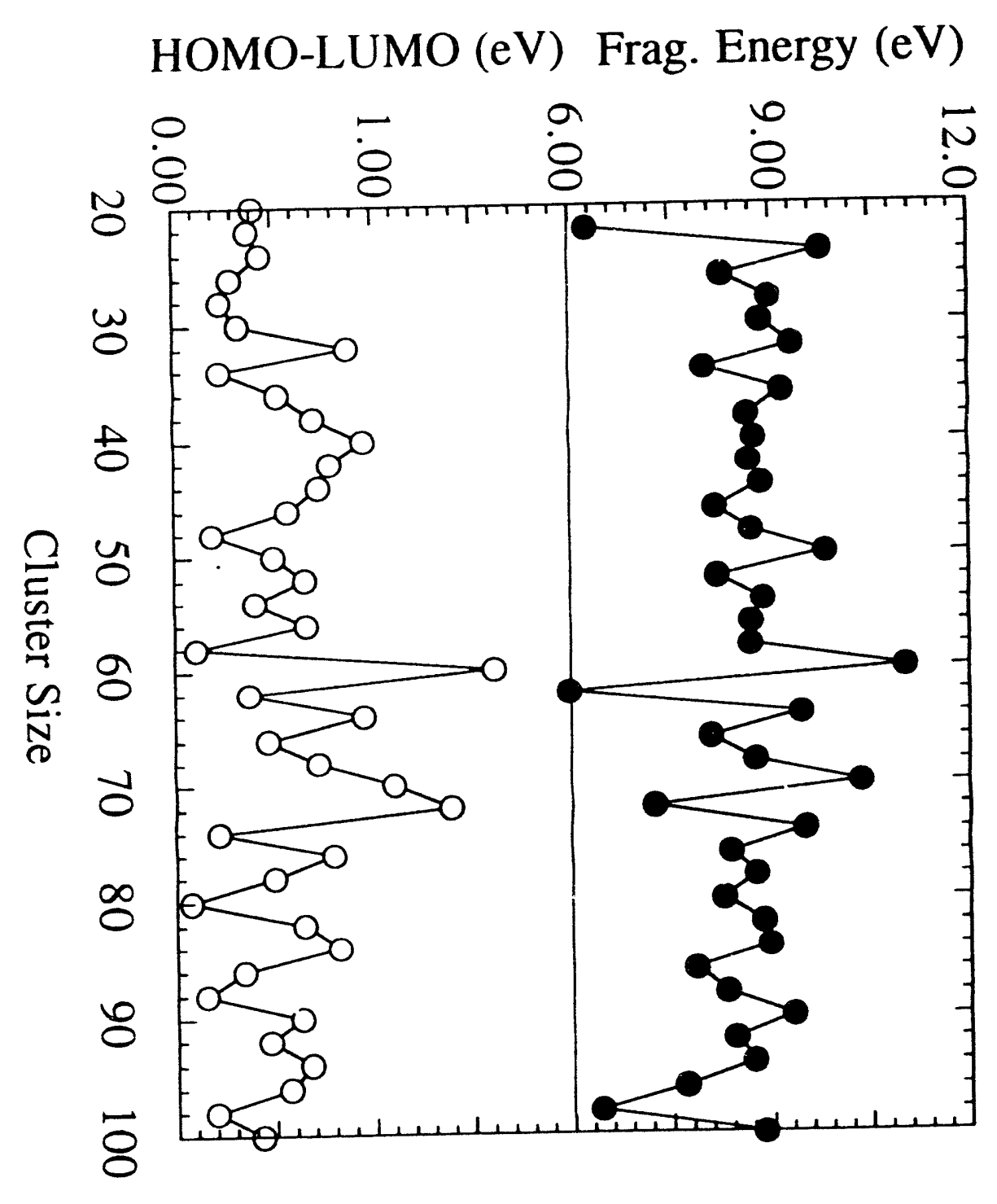


isomers with energies very close to that of ground-state isomer. This may lead to the possibility that several isomers can coexist in synthesis for a given cluster size. From this systematic study, we found that the superstable carbon clusters not only have high fragmentation stability but also have a large HOMO-LUMO energy separation. On the contrary, the fullerenes which are hard to obtain in experiments have a tendency towards fragmentation or further chemical reaction. 


\section{CHAPTER 7. THREE DIMENSIONAL CARBON NETWORKS}

\section{Introduction}

The recent discovery and ability to produce fullerenes in macroscopic quantities highlights the new phase of carbon which has cage structures with coordination number 3. If we view a fullerene molecule as composition of polygons of $\mathrm{C}$-atoms joined together by nearest neighbor bonds, the geometrical structure of fullerenes can be summarized in one single equation:

$$
\sum_{p}(6-p) M_{p}=12
$$

where $M_{p}$ is the number of p-polygons (e.g. $M_{6}$ is the number of hexagons). Equation 7.1 is the consequence of two and only two conditions: (1) the structure satisfies the Euler equation for an object topologically equivalent to a sphere, which says $F+V$ $=\mathrm{E}+2$, where $\mathrm{F}$ is the number of faces $\left(F=\sum_{p} M_{p}\right), \mathrm{V}$ is the number of vertices ( $F=$ no. of carbon atoms), and $E$ is the number of edges $(E=$ no. of nearest neighbor bonds); (2) all carbon atoms are 3 -fold coordinated (i.e. $E=3 \mathrm{~V} / 2$ ). This equation imposes no restriction on the number of hexagons, which is not surprising since in the hexagonal arrangement, the carbon atoms are "naturally" 3 -fold coordinated. Note that Equation 7.1 can be satisfied by many different combinations of polygons, but if we restrict ourselves to one type of polygon other than hexagons, we have 


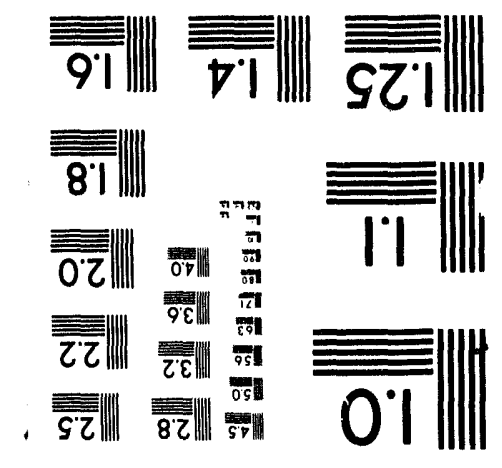



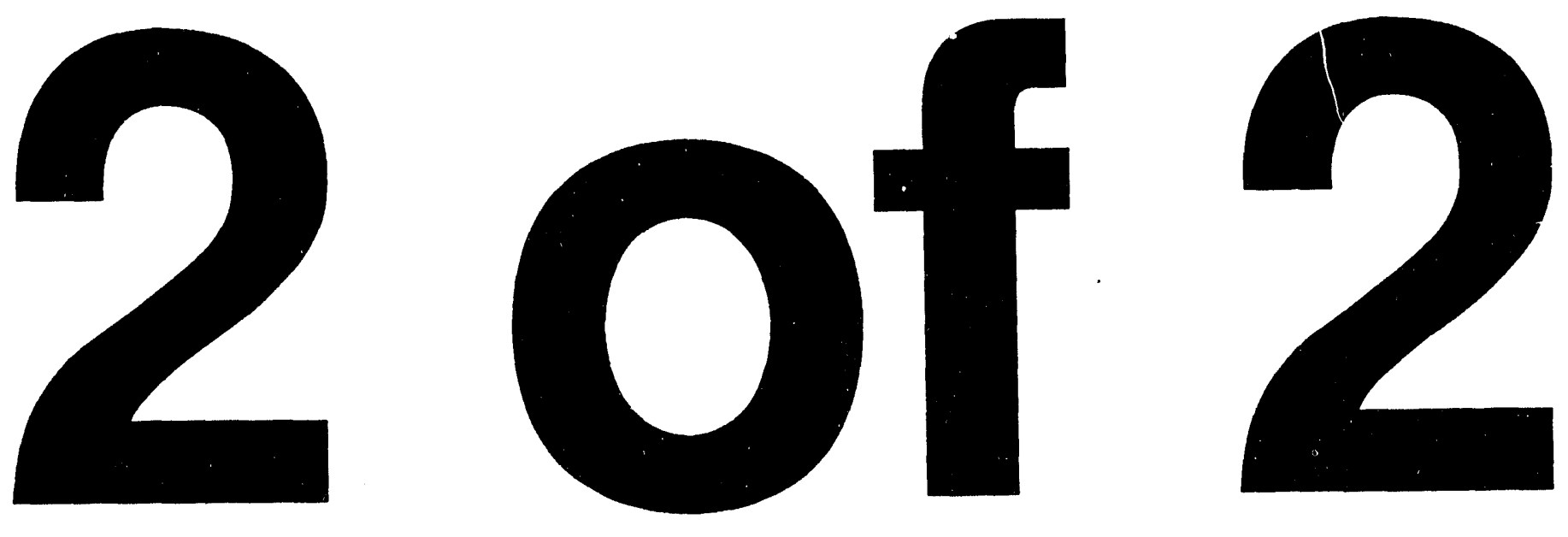
only one choice: 12 pentagons. This apparently is what nature favors, as a large body of experimental and theoretical evidence suggests. The Euler equation can be generalized from a spherical surface to

$$
F+V=E+X
$$

where $X$ is the Euler Characteristic of the surface, and $X=2-2 g$, where $g$ is the "genus", or the number of holes bored through the sphere. A sphere has $g=0$, and an object topologically equivalent to a torus (donut) has $g=1$. In this case, Equation 7.1 is generalized to

$$
\sum_{p}(6-p) M_{p}=12-12 g
$$

For $\mathbf{g}=1$, where the carbon atoms are on the surface of a torus,

$$
\sum_{p}(6-p) M_{p}=0
$$

which can be satisfied by hexagons only. This interesting possibility has already been realized in the form of "carbon tubules" [53], since we can view a tube as a section of a torus of very large radius.

It is interesting to consider the case for $g>1$. A molecule with the carbon atoms sitting on a surface that is a connected sum of $n$ tori can be constructed. If we imagine that this huge molecule grows without bound in all 3 directions by adding more and more tori, a true three-dimensional (3D) network of carbon with coordination number three will be formed. This interesting possibility was first considered by Mackay and Terrone [116], who decorated periodic minimal surfaces with 3 -fold coordinated carbon atoms. Structures that are energy minimized using simple empirical potentials are discussed in two recent papers, and the resulting structures are called "negative- 
curvature-fullerenes" by Vanderbilt and Tersoff [117] and "Schwarzites" by Lenosky et al. [118].

It is evident from Equation 7.3 that once $g>1$, it is necessary that the system contains p-membered rings with $p>6$. So if we view fullerenes and Schwarzites as defective graphite, the defects in the fullerenes are the pentagons, which induce the positive curvature and the defects in Schwarzite are heptagons, octagons or higher member rings that induce the negative curvatures in the carbon sheets.

\section{Search Scheme}

The fullerene search scheme described in the last chapter can be extended to three dimensional periodic systems to search for low-energy networks for certain types of periodic surface. The simplest case is the simple cubic lattice and one structure, P216, proposed by Phillips et al. [119] contains 216 atoms per unit cell with sixand seven-membered rings only. If we look carefully, the carbon network spanned in the three dimensional space is very similar to the Fermi surface of the simple cubic lattice. The equation of this surface is:

$$
E=b(\cos (a x)+\cos (a y)+\cos (a z))
$$

For a simple cubic lattice, each lattice point has six nearest neighbors which correspond to $\mathrm{g}=3$. Then from Equation 7.3, the number of heptagons is 24 if there are no other higher polygons. Then for 216 atoms, it is easy to get the total number of faces, 80 hexagon and 24 heptagons, from Equation 7.2. Initially, these 104 points were randomly distributed in the unit cell and allowed to interact with each via a 
repulsive potential $1 / \mathrm{r}^{6}$. There is also an external potential:

$$
U=-E^{2}, \quad E=b(\cos (a x)+\cos (a y)+\cos (a z))
$$

to confine these points on the $\mathrm{P}$ surface according to Equation 7.5.

\section{Structural, Electronic and Vibrational Properties}

The resultant network was then converted to an atomic network and we got exactly 80 hexagons and 24 heptagons as we expected. However, the new network NP216 is not the same as P216 generated by Phillips et al. [119] and our new structure has a larger binding energy and lower symmetry. The network NP216 is shown in Fig. 7.1 along with network P216 in Fig. 7.2 for purposes of comparison. One interesting observation is that our new model has paired heptagons while the heptagons in the P216 network are all isolated, which seems contrary to the isolated-pentagon rule for fullerenes. This was also noticed by Townsend et al. [120] and it may due to the geometrical difference between fullerenes and Schwarzites. For Schwarzites, the network can be roughly divided into "ball" and "joint" regions with positive and negative curvatures respectively. To reduce the strain energy, the heptagons (providing negative curvature) are better placed at these negative curvature regions (necks of the joints). Then 24 heptagons are restricted in a relatively small region and they fuse together. For fullerene, there is only the "ball" region and the pentagons can be distributed everywhere on the surface. For the P216 structure, we notice that some of the heptagons are misplaced at the "ball" region and that causes more strain energy. However, as the number of atoms per unit cell increases, the area of the "joints" becomes large while the number of heptagons is still the same. We anticipate that 
the "isolated-heptagons" structures will be favoured.

The binding energies of various networks are listed in Table 7.1 and results of other calculations are included for comparison. The gaps and densities of various networks are listed in Table 7.2 and here the gap is defined as the energy difference between the highest occupied orbital and lowest unoccupied orbital at $k=0$ for the fully relaxed structures. Since these carbon networks have large unit cells and relatively small curvatures, a small dispersion in the band structure is expected [119]. As for the case of NP216, we have sampled up to $300 \mathrm{k}$-points in the reduced Brillouin zone and the gap is narrowed by only $0.1 \mathrm{eV}$. The bulk modulus of the NP216 network is $1.25 \mathrm{Mba}$ in comparison to regular metals; Fe 1.68 Mbar, Cu 1.37 Mbar and Ti 1.05 Mbar. By considering the density of NP216, which is only about one seventh of the iron, this carbon network is very hard material.

Another interesting question we would ask ourself is: is the value 216 special? In the fullerene family, $\mathrm{C}_{60}$ is not special in the sense of binding energy since every fullerene larger than 68 has a larger binding energy [47]. But if we consider the proposed growth mechanism $[114,115]$, fullerenes can grow by adding two carbon atoms each time or can shrink by losing two carbon atoms, then only the stability of a fullerene relative to its neighbours really matters. In our previous study [47], we did find that $\mathrm{C}_{60}, \mathrm{C}_{70}$ and $\mathrm{C}_{84}$, the three most abundant fullerene members, are more stable than their neighbors. Though the growth mechanism of the carbon network is still not clear, by analogy to the fullerene, we searched the possible structures of networks with 214 atoms and 218 atoms per unit cell for the $P$ surface. We found that 216 is indeed a magic number relative to its neighbors. (see Table 7.1).

There was speculation that these hypothetical networks may already exist in the 


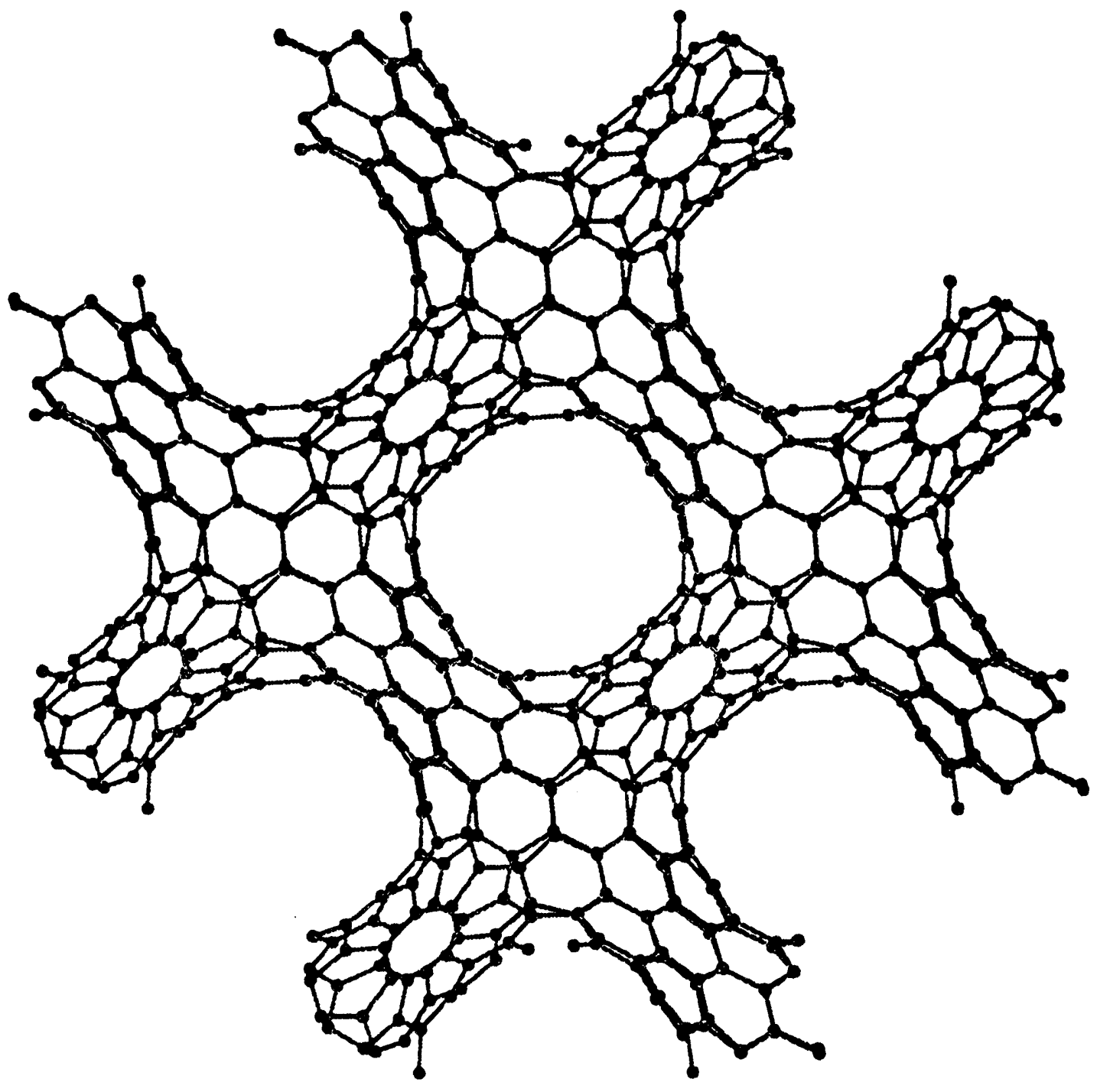

Figure 7.1: The ball and stick model of new generated NP216 network. The unit cell is expanded to six nearest neighbour in simple cubic lattice and the total number of atoms in the figure is 864 . 


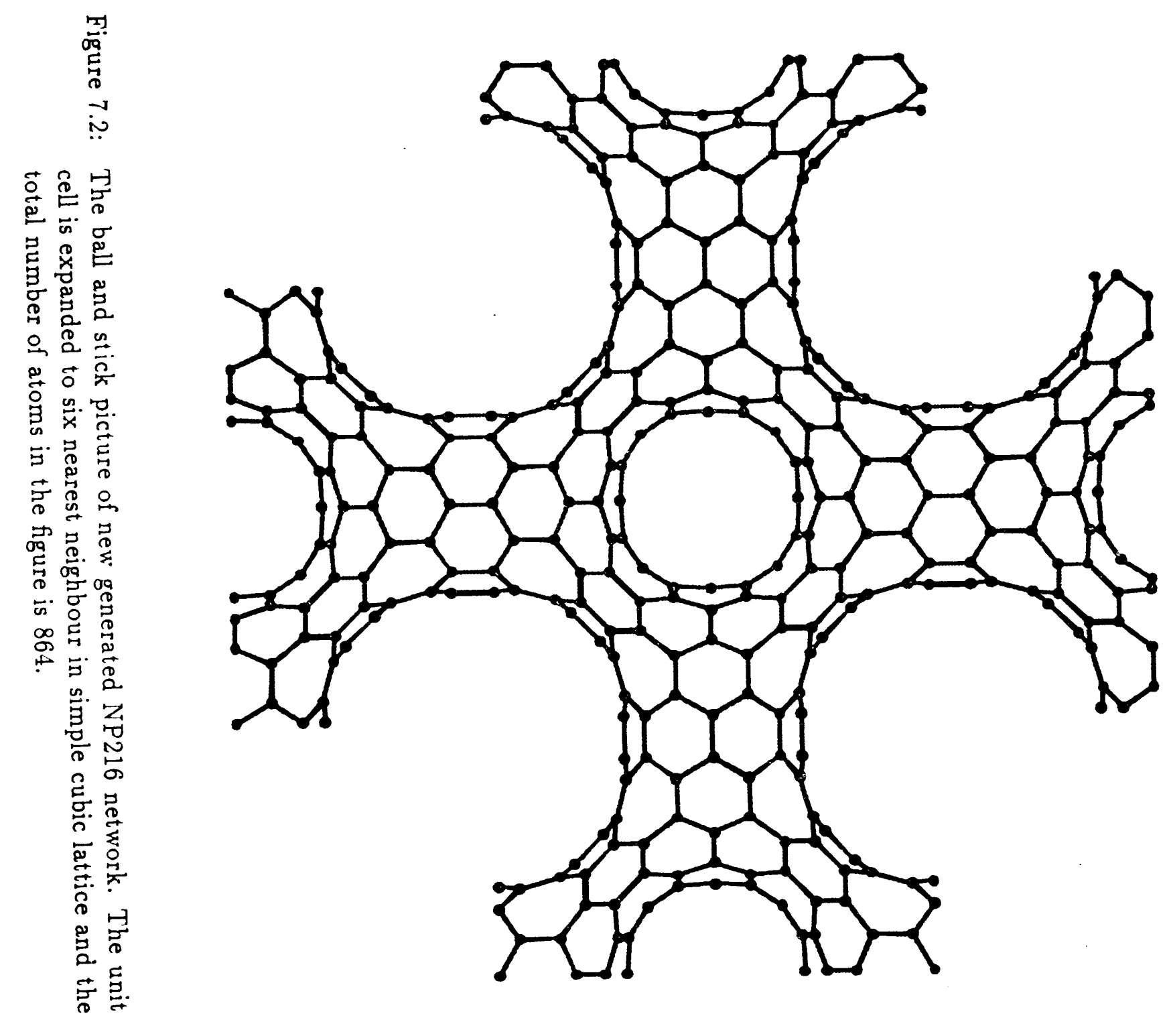


Table 7.1: Binding energies of three-dimensional carbon networks relative to graphite in the unit of $\mathrm{eV}$ per atom.

\begin{tabular}{cccc}
\hline Network & Tight-Binding & Ref. 14 & Ref. 5 \\
\hline P216 & 0.210 & 0.212 & 0.197 \\
D216 & 0.214 & 0.216 & 0.182 \\
P192 & 0.189 & 0.193 & 0.186 \\
D168 & 0.248 & 0.249 & 0.218 \\
NP216 & 0.181 & $\ldots$ & $\ldots$ \\
NP218 & 0.212 & $\ldots$ & $\ldots$ \\
NP214 & 0.194 & $\ldots$ & $\ldots$ \\
C 60 & 0.401 & $\ldots$ & $\ldots$ \\
\hline \hline
\end{tabular}

Table 7.2: Gaps $(\mathrm{eV})$ and densities $\left(\mathrm{g} / \mathrm{cm}^{-3}\right)$ of three-dimensional carbon networks.

\begin{tabular}{ccc}
\hline Network & Gap & Density \\
\hline P216 & 1.08 & 1.03 \\
D216 & 0.95 & 1.15 \\
P192 & 1.01 & 1.17 \\
D168 & 0.99 & 1.28 \\
NP216 & 0.56 & 1.11 \\
NP218 & 0.16 & 1.10 \\
NP214 & 0.02 & 1.14 \\
\hline \hline
\end{tabular}


carbon soot [120], then the difficulty is how to detect the existence of these novel materials. Due to the low symmetries of these Schwartzite, the Raman and infrared spectra will be very complicated. One appropriate experimental tool can be neutron scattering which measures the phonon density of states by picking up all vibrational modes.

We have calculated the phonon density of states by solving the dynamic matrix and it is plotted in Fig. 7.3. For comparison, the phonon density of states of graphite and diamond are also calculated. To verify the reliability of our calculations of the phonon density of states, we plotted the experimental results for diamond (Fig. 7.3(b)) [121]. As we can see the agreement between our calculation and the experimental measurement is excellent. Since the coordination number of NP216 is three, the same as that of graphite, we will focus on the difference in the phonon density of states of these two structures. In general, the spectrum of the NP216 network is shifted to the lower end by about $6 \mathrm{meV}$. Since neutron scattering has quite high resolution around 50-100 meV, the cleat shift of the dip (marked as A in Fig. 7.3(a)) could be detected. Another usual carbon form existing in soot is amorphous carbon. But for amorphous carbon the phonon density states spectrum has no dip [122], so it is very easy to be distinguished from NP216 network.

\section{Summary}

We have calculated the energies, electronic band gaps and densities of various three-dimensional carbon networks. By using a face-dual network scheme, we also propose a new P-type network which has the largest binding energy ever found. The band gap and the bulk modulus of this new network indicate a possible brand 


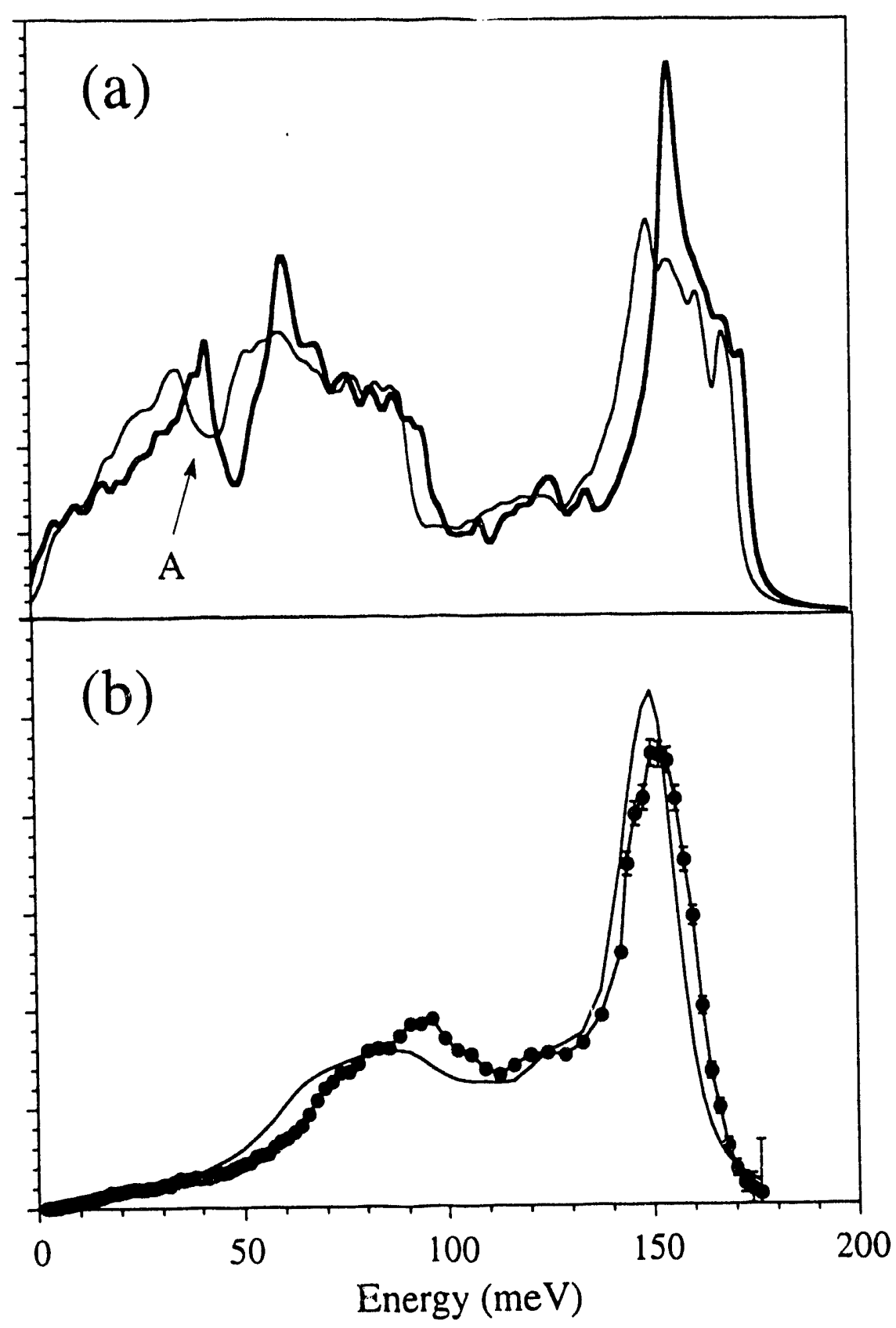

Figure 7.3: (a) The phonon density of states of NP216 network (thin line) and graphite (thick line). (b) The theoretical calculation (line) and experimental measurement (dots with corresponding error bar) of phonon density of states of diamond. 
new semiconductor material. More importantly, we have calculated the vibrational density of states of this new network and it exhibits several distinguishable feature compared with graphite and amorphous carbon. So it may provide a useful clue for experimental search of this kind of novel carbon form. 


\section{CHAPTER 8. THERMAL DISINTEGRATION OF FULLERENES}

\section{Introduction}

In this Chapter, we present a systematic study of the stability of the carbon fullerenes against thermal disintegration. This is a first step towards establishing a "phase diagram" for the fullerenes as a function of cluster size and temperature. Since the fullerenes are produced at very high temperatures, such as arc burning or laser vaporization of graphite, this knowledge is very useful for controlling the buckyball synthesis process. On the other hand, fullerenes possess a cage like structure which spreads all carbon atoms over a closed surface. It is a system with both three and two dimensional characters. Therefore, understanding the process of thermal disintegration of this unique system is also of interest from a basic scientific point of view. In our present simulations, we are studying systems at fairly high temperatures. To include the effects of the electronic states at finite temperatures, we used the Fermi-Dirac distribution function (FDDF) to describe the occupation of the electronic states in the energy and force calculations in our tight-binding molecular dynamics (TBMD) simulations. Recently, the FDDF has been used to simulate the metallic

system in first-principles molecular dynamics (FPMD) simulations [89, 90, 91]. This implementation not only avoids the instability due to the change of occupancies near the Fermi level but also includes the electronic entropy. Details of the method have 
been discussed in Chapter 5. In this Chapter, we study the effect of the electronic temperature on the thermal disintegration of fullerenes.

\section{Details of Simulations}

Constant-temperature molecular dynamics simulation is performed using simple velocity scaling for temperature control. The starting configurations are the groundstate fullerene structures obtained from our previous work $[48,49]$. We study only the even-numbered clusters since odd-numbered clusters are observed to be much less abundant in the experimental mass spectra. Starting with the cluster in the groundstate fullerene structure, we gradually heat up the system at a rate of $500 \mathrm{~K}$ per 2000 steps $\left(0.7 \times 10^{-15}\right.$ second per step $)$. When the system is close to the fragmentation point, we reduce the heating rate to $100 \mathrm{~K}$ per 2000 steps in order to have a more accurate estimation of the fragmentation temperature. The potential energy of the system is monitored as a function of temperature, the results of a typical run is shown in Fig. 8.1. During the heating up process, there is a sudden jump in the potential energy at a certain temperature. By monitoring a graphic display of the atomic motions during the simulation, we verified that this corresponds to the onset of bond-breaking in the fullerene cage structure. This temperature is then recorded as the fragmentation temperature of the carbon fullerene. For $\mathrm{C}_{60}$, we also repeated the simulation using stochastic temperature control and obtained almost the same fragmentation temperature (within $100 \mathrm{~K}$ ) as the simple velocity scaling method.

In our previous TBMD simulations $[46,47,48,49]$, the electrons always occupy

the lowest energy states. In the other words, the electron temperature $\mathrm{T}_{e l}$ is set to zero. This treatment is acceptable when we are searching for the ground-state 


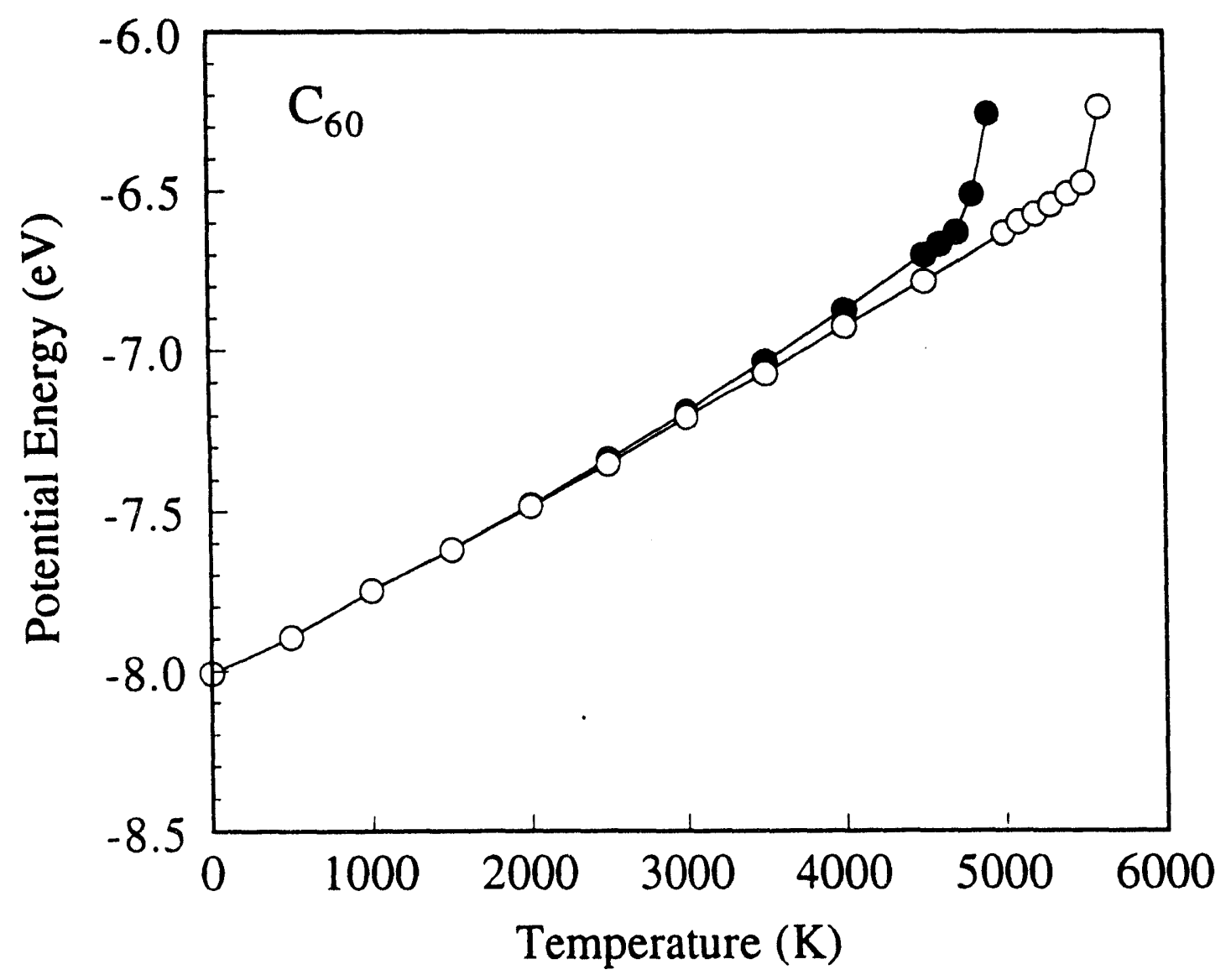

Figure 8.1: The potential energy (eV/atom) of $\mathrm{C}_{60}$ is plotted as function of temperature. Where the solid dots are the results with F-D distribution and the open dots are the results without F-D distribution. 
geometries when the temperature of the system is finally cooled down to zero. For carbon clusters with relatively large HOMO-LUMO gaps we expect this to be an accurate description at low temperatures. But in the present study, the clusters are warmed up to several thousand degrees. In the high temperature regime, the F-D distribution is important because of the higher electronic temperature and narrower HOMO-LUMO gap caused by thermal vibrations. For $\mathrm{C}_{60}$, the HOMO-LUMO gap drops from $1.6 \mathrm{eV}$ at $\mathrm{T}=0$ to $0.2 \mathrm{eV}$ at the fragmentation temperature which is about $5000 \mathrm{~K}\left(k_{B} T \sim 0.43 \mathrm{eV}\right)$.

\section{Results and Discussion}

The results of the melting temperature as a function of cluster size ranging from $\mathrm{C}_{20}$ to $\mathrm{C}_{90}$ is plotted in Fig. 8.2(a). The heat of formation of these fullerenes relative to graphite is also plotted for the purpose of comparison (Fig. 8.2(b)). As a general trend, we find that the melting temperature increases almost linearly with cluster size in the range $\mathrm{C}_{20}$ to $\mathrm{C}_{58}$. This trend is quite different from that of the formation energy in which the slope decreases rapidly as the cage size increased. When the cluster size passes 60 , the melting temperature of the larger fullerenes $(n \geq 60)$ is almost a constant, although energetically their stability keeps on increasing. The deviation of the melting temperature curve from the heat of formation curve is easy to understand since the former measures the weakest bond of the cage, while the later is a measure of the average bonding in the cluster. Choosing $\mathrm{C}_{90}$ as an example, even though it has a larger binding energy than $\mathrm{C}_{60}$ as a whole molecule, it has certain bonds weaker than others due to its low-symmetry $\left(D_{2}\right)$ structure. These weak bonds are not stronger than that of $\mathrm{C}_{60}$ and that is why the melting temperatures of $\mathrm{C}_{90}$ 
and $\mathrm{C}_{60}$ are almost the same. Finally, it is worth noting that a sharp kink is found at $\mathrm{C}_{60}$ in both energy and melting temperature plots, indicating its enhanced stability relative to its neighbours.

The criterion we used to determine the fragmentation point in this study may have overestimated the fragmentation temperature due to the rather short simulation times practicable in TBMD simulations. From our simulation, the time for redistributing the added energy among the different degrees of freedom is estimated to be about 5000 to 6000 steps. Our heating rate of $100 \mathrm{~K}$ per 2000 steps near the disintegration region may therefore overestimate the disintegration temperature by about $200 \mathrm{~K}$ (The faster heating rate of $500 \mathrm{~K}$ per 2000 steps far below the critical temperature will not have any significant effects on the disintegration temperature as long as the system is sufficiently thermalized at temperatures just below the disintegration temperature). We have also performed longer time simulations for the thermal disintegration process of $\mathrm{C}_{60}$. We found that if we perform the simulation at $200 \mathrm{~K}$ lower than the disintegration temperature determined from the short simulation time (i.e., 2000 steps or 1.4 ps), the buckyball will break after 14000 steps (9.8 ps) of simulation. However, if we lower the temperature of the system by another $100 \mathrm{~K}$, the $\mathrm{C}_{60}$ molecule is found to be stable up to $50 \mathrm{ps}$ of simulation time. This result is consistent with the above analysis based on the energy redistribution process.

It should also be kept in mind that the present empirical TB model is fitted to the binding energy of LDA results which generally overestimates the binding energy of graphite by about $10 \%$ in comparison with the experimental value. Thus there exists a corresponding systematic overestimate of the melting temperature if one wants to make a direct comparison with experimental results. Also, in the real 

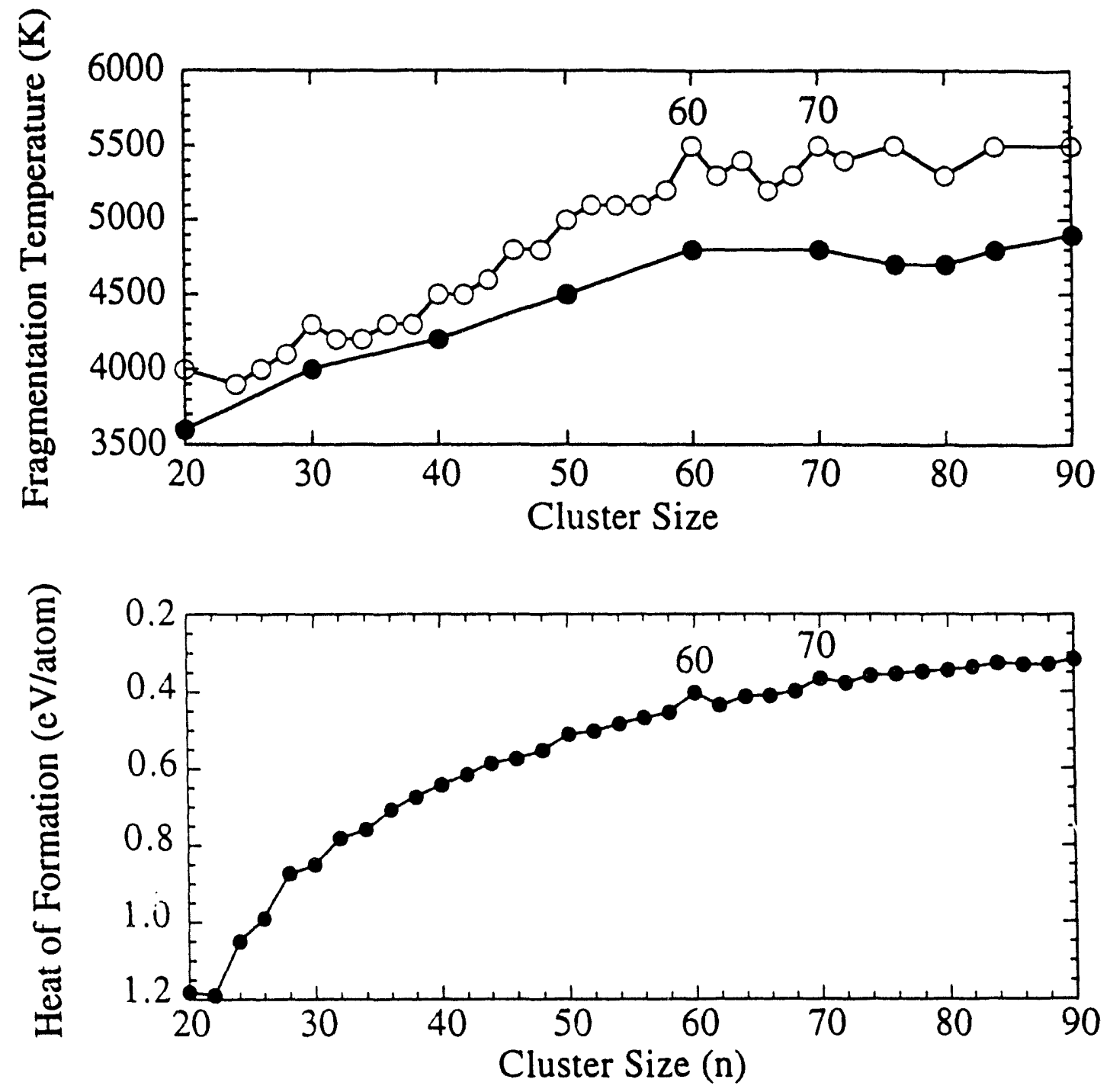

Figure 8.2: Fragmentation temperature of fullerene is plotted as function of cluster size. The solid dots are the results with the F-D distribution and the open dots are the results without the F-D distribution. For purposes of comparison, the heat of formation of fullerenes is plotted in the lower portion. (Note: the heat of formation is relative to graphite in units of $\mathrm{eV}$ per atom). 
experimental condition, the disintegration process can be influenced by collisions with other objects, such as atoms, dimers and cluster fragments.

A schematic illustration of the fullerene fragmentation process is plotted in Fig. 8.3. In a typical disintegration process, after the onset of bond breaking, the atoms with dangling bonds stick out from the cage surface. These off-surface atoms vibrate much more violently than the rest of the network and the whole cage structure is finally be unravelled into a ring or multiple-ring structure with the breaking off of fragments like dimers.

There is no noticeable difference in the disintegration process for simulations with and without including the F-D distribution for the occupancy of electronic states except for a lowering of the fragmentation temperatures. As the electrons partially occupy the states with anti-bonding character above the Fermi level, the bonds between carbon atoms become weaker and the clusters break apart more easily. For $\mathrm{C}_{60}$, the HOMO-LUMO gap at $\mathrm{T}=0 \mathrm{~K}$ is $1.6 \mathrm{eV}$, corresponding to $18500 \mathrm{~K}$, thus the occupancy above the Fermi level is negligible. But as the temperature increases, the structural distortions due to thermal vibrations cause the HOMOLUMO gap to decrease rather quickly. The HOMO-LUMO gaps plotted in Fig. 8.4 are obtained from an average of 2000 time steps from the constant temperature simulations. We can see that HOMO-LUMO gap drops to around $0.25 \mathrm{eV}$ near the fragmentation temperature $5000 \mathrm{~K}$. In Fig. 8.1, we show the potential energy of $\mathrm{C}_{60}$ as function of temperature for the simulations with and without the FermiDirac distribution. Since $\mathrm{C}_{60}$ has a large HOMO-LUMO gap, these two curves match closely for low temperatures. But after the temperature exceeds $2500 \mathrm{~K}$, there is a significant difference between the two curves. The curve with zero electronic 
(a) $t=0 \mathrm{ps}$

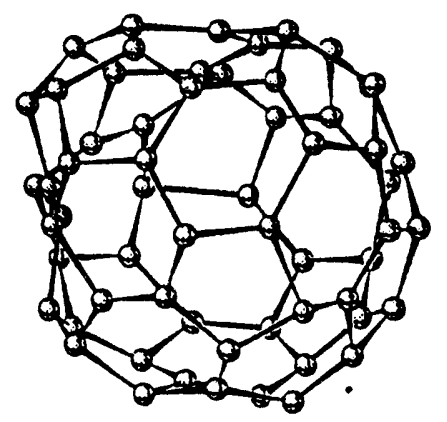

(c) $\mathrm{t}=1.40 \mathrm{ps}$

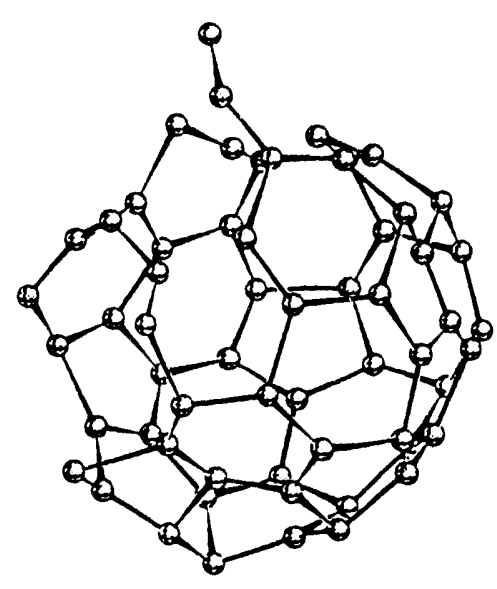

(b) $\mathrm{t}=0.07 \mathrm{ps}$

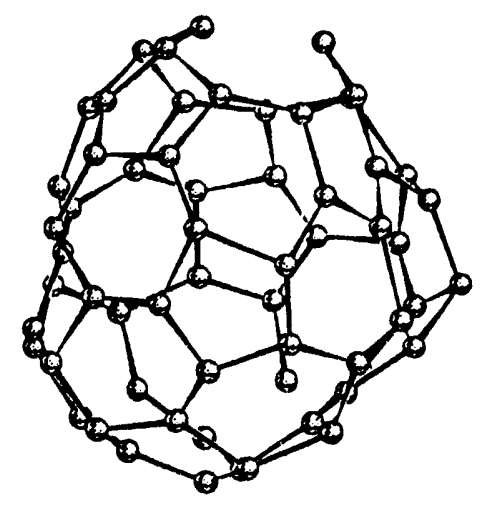

(d) $\mathrm{t}=2.50 \mathrm{ps}$

8

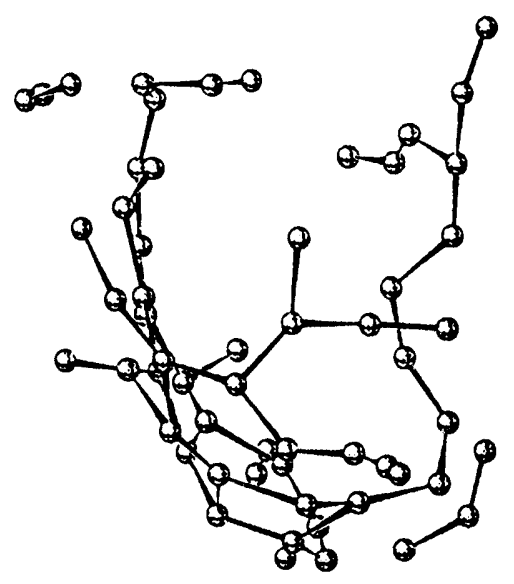

Figure 8.3: The selected snapshots of $\mathrm{C}_{60}$ fragmentation process at $\mathrm{T}=5500 \mathrm{~K}$. 
temperature is almost linear, indicating nearly harmonic thermal vibrations, while the curve with non-zero electronic temperature increases faster. Thus temperature effects for the electronic system enhances the anharmonic effects at high temperatures and this may also affect other dynamical properties, such as shifts and broadening of the vibration frequencies of the molecule. In the present study, the effect on fragmentation temperatures is less than $10 \%$ because of the relatively large HOMOLUMO gaps in mosi of the carbon clusters.

\section{Summary}

In summary, we performed tight-binding molecular dynamics simulations of the thermal disintegration of fullerene cages. We found that the fragmentation temperature is sensitive to the cluster size for small clusters but almost constant for larger clusters. With the implementation of the Fermi-Dirac distribution for the occupation of the electronic states, the fragmentation temperatures of carbon fullerenes are reduced by about $10 \%$ due to the occupation of anti-bonding states at $T_{e l} \neq$ 0 . From this study, we found that the Fermi-Dirac distribution of electronic states also enhances the anharmonicity of the carbon clusters and this might be important for studying the dynamics of systems that have smaller or no band gaps at high temperatures. 


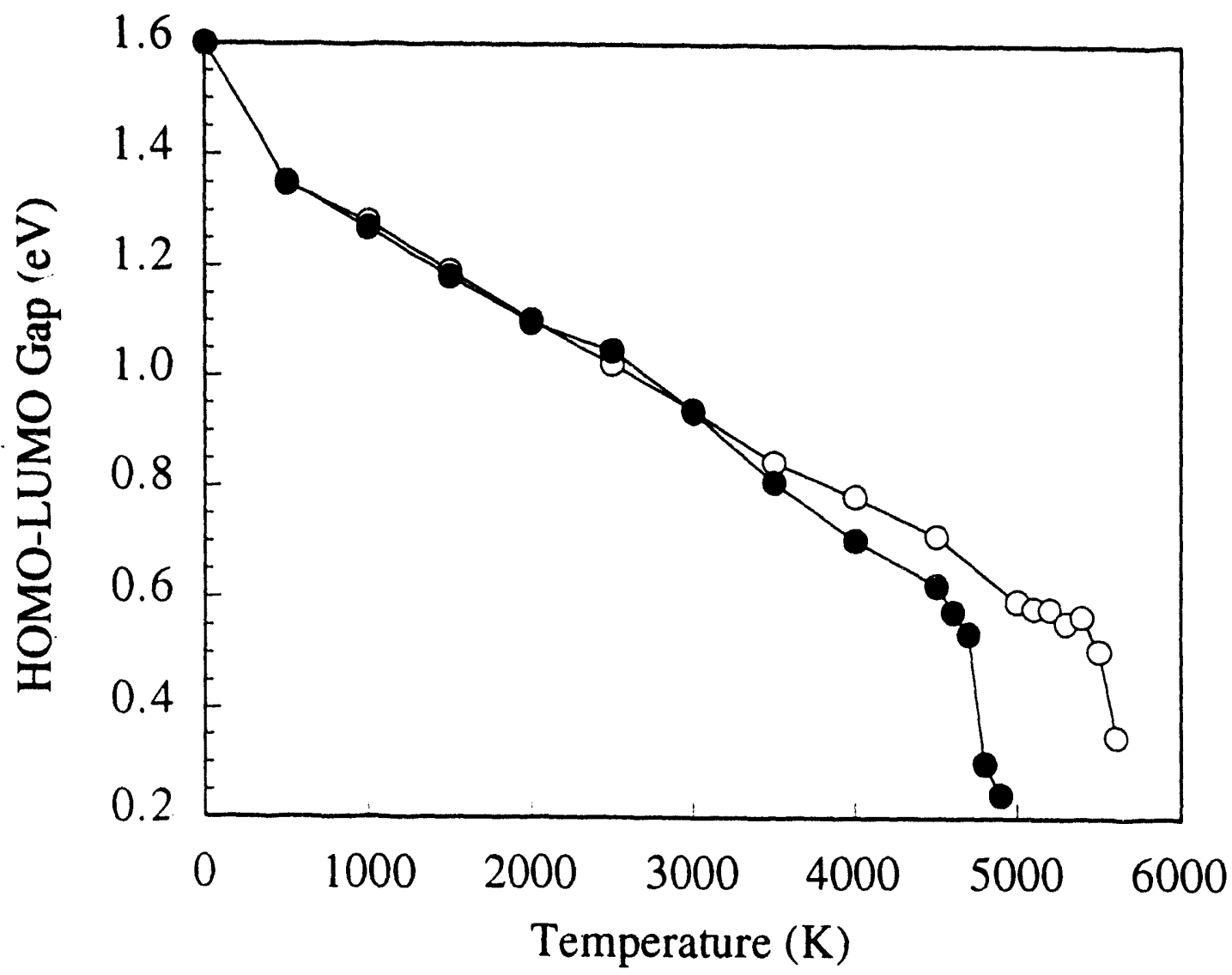

Figure 8.4: The HOMO-LUMO gap of $\mathrm{C}_{60}$ is plotted as function of temperature where the solid dots are the results with the F-D distribution and the open dots are the results without the F-D distribution. 


\section{CHAPTER 9. COLLISIONS OF FULLERENES}

\section{Introduction}

The success in producing macroscopic quantities of $\mathrm{C}_{60}$ led to a rapid surge in interest from scientists in many areas. In a short period, we learned a lot about many interesting properties and potential applications of this novel material [30]. However, the detailed fullerene formation process is still a mystery. It was realized that the high yield of $\mathrm{C}_{60}$ in the experimental synthesis is not solely due to energy stability, otherwise $\mathrm{C}_{70}$ should be more abundant than $\mathrm{C}_{60}$ since it has a larger binding energy per atom $[46,108]$. In order to explain the high yield of $\mathrm{C}_{60}$, one has to invoke kinetic factors and postulate that the $\mathrm{C}_{60}$ molecule has a high barrier towards both further growth or fragmentation under the experimental conditions $[123,50]$.

In this context, it is of interest to study the behavior of the $\mathrm{C}_{60}$ molecule under collisions. The stability of the $\mathrm{C}_{60}$ molecule under high energy collisions is also an important consideration in some potential app.ications. Furthermore, understanding the $\mathrm{C}_{60}$ collision process will help us predict the behavior of solid $\mathrm{C}_{60}$ under sudden shock compression when cluster collisions occur in the solid state.

In the earliest carbon cluster collision experiment, Callahan et al. [124] accelerated $\mathrm{C}_{60}^{2+}$ ions to collide with a polished stainless steel surface. They found that the buckyball structure can survive up to a collision energy of $120 \mathrm{eV}$ per molecule. 
Experiments were also performed for surface-scattering of $\mathrm{C}_{60}$ ions against a graphite surface $[125,126]$. No impact-induced fragmentation was observed for impact energy as high as $200 \mathrm{eV}$ per molecule. More recently, collisions between carbon clusters were studied in experiments [128]. When the collision energy is increased to $400 \mathrm{eV}$ per molecule, heavy products, such as $C_{90}^{+}$and $\mathrm{C}_{120}^{+}$, were observed in addition to fragments smaller than $\mathrm{C}_{60}$.

Theoretical simulations of collisions of neutral $\mathrm{C}_{60}$ molecules with a H-terminated diamond (111) surface has been reported by Mowrey et al. [129]. They found that while the collisions are highly inelastic, the $\mathrm{C}_{60}$ molecules retain their cage structures after bouncing back from the diamond surface even though they are severely deformed. Collisions between carbon clusters have also been studied by Ballone and Milani [130] and by Seifert and Schmidt [131].

In this Chapter, we present a molecular-dynamics (MD) simulation study of the behavior of the $C_{60}$ molecule when it collides with another $C_{60}$ molecule at high kinetic energies.

\section{Simulations of Fullerene Collisions}

Simulations of collisions between $\mathrm{C}_{60}$ molecules are performed at three temperatures: $0 \mathrm{~K}, 2000 \mathrm{~K}$ and $3000 \mathrm{~K}$. The time step in our simulation is $7 \times 10^{-16}$ second and the total energy is conserved to within $10^{-5} \mathrm{eV} /$ atom during 1500 steps for each trajectory. For each temperature, the results of head in collisions were observed over a wide range of collision energies. At zero temperature, several special orientations of colliding molecules have been investigated: (a) pentagon to pentagon, (b) bond to bond (perpendicular), (c) bond to bond (parallel) and (d) hexagon to hexagon 
which are shown in Fig. 9.1. Collisions between $\mathrm{C}_{30}$ clusters were also studied for comparison with the results of $\mathrm{C}_{60}$. The $\mathrm{C}_{60}$ and $\mathrm{C}_{30}$ molecules used in our studies are the ground-state isomers with $\mathrm{I}_{h}$ and $\mathrm{C}_{2 v}$ symmetry respectively.

For high temperature collisions, the orientations of $\mathrm{C}_{60}$ molecules and the velocity of individual atom are random. Simulation of single collisions is not enough to reflect the significance of their statistical nature. Here, we have utilized parallel computers to simulate the different trajectories simultaneously. The system is first warmed up to the desired temperature and allowed to equilibrate for sufficient time. Starting from randomly chosen configurations, we simulate the trajectories of different collisions on different nodes of parallel computer. Since there is no information exchange between nodes during the simulations, the program is fully parallel. For each specific collision energy and temperature, sixteen trajectories were simulated to get the average value and standard error.

In the simulation of zero temperature collisions, the fully relaxed clusters are initially separated by a distance beyond the interaction range of our tight-binding potential and allowed to approach each other with equal speeds (determined by the collision energy) so that the center of mass remains unchanged. For the high temperature collisions, the procedure is the same except that the clusters are warmed up before starting the collision process. All the collisions are simulated with zero impact parameter corresponding to head-on collisions.

\section{Results and Discussions}

For all the cases we have studied, there are qualitatively three distinct regimes as the collision energy increases: At low collision energies, the clusters deform upon 
(a)
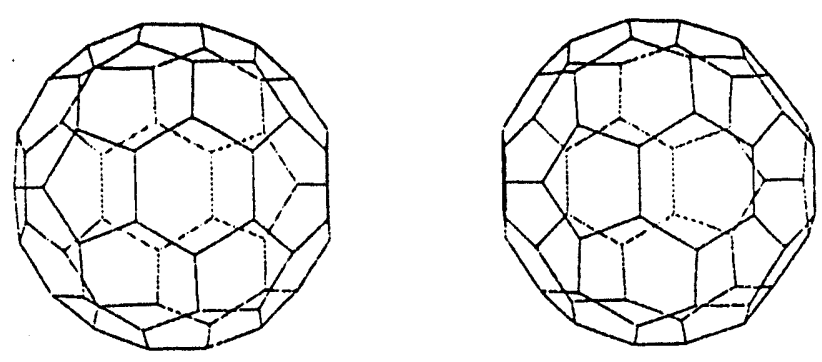

(b)
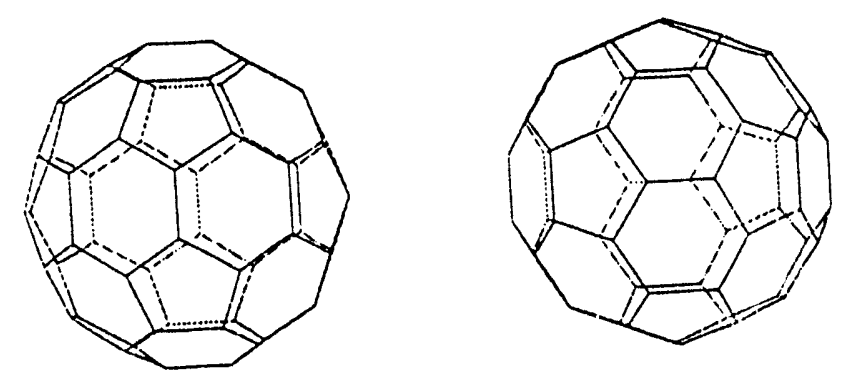

(c)
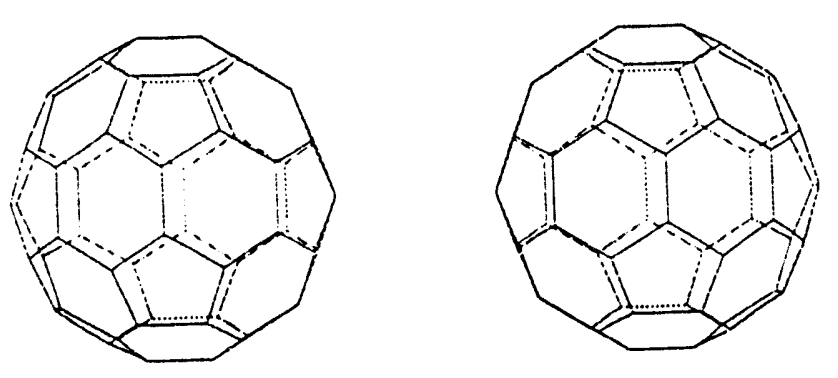

(d)
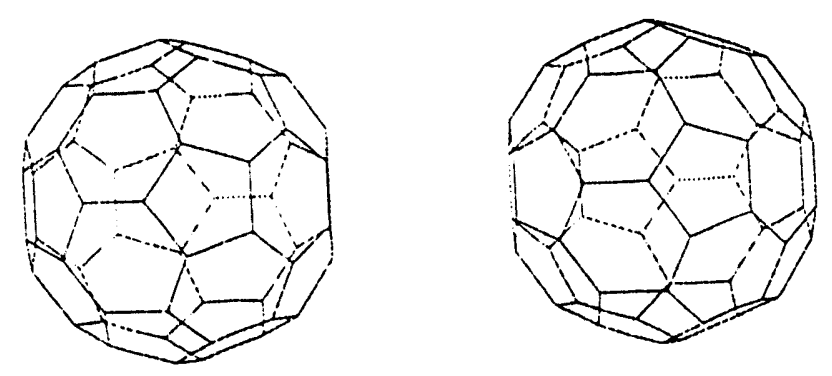

Figure 9.1: The schematic orientations of collisions of two $\mathrm{C}_{60}$ balls: (a)The pentagon to pentagon. (b)bond to bond (perpendicular). (c) bond to bond (parallel). (d) hexagon to hexagon. 
contact and bounce back with their cage structures intact. After collision, a fraction of the translational kinetic energy of the cluster is converted to intra-molecular vibrations, notably in the breathing vibrational mode. At higher collision energies, some carbon bonds are broken and there are atoms with dangling bonds. After impact, if atoms from different molecules form bonds with each other, then the molecules will stick (fuse) together. In most cases, the new cluster is not a perfect fullerene and usually contains dangling bonds. These "poor" clusters are energetically quite unstable and it should be quite easy for them to grow in subsequent collisions with other clusters or small grains existing in the carbon gas phase in the process of soot formation. If the kinetic energy is even larger, the collision will reach the third regime in which the clusters will break up into smaller pieces. The most commonly observed fragments are carbon dimers, trimers, linear chains and multiple rings. The schematic views of these three stages are shown in the Fig. 9.2.

The critical collision energies for the occurrence of fusion and fragmentation are listed in Table 9.1 and Table 9.2. For a given collision energy, we perform sixteen collisions for the high temperature clusters. The critical energies indicate the energy at which fusion (or fragmentation) occurs in at least one of the sixteen trials. Since thermal vibrations weaken the carbon bonding of the cage, we observe a decrease in the critical energies as the temperature increases.

From Table 9.1 and Table 9.2 , we can see that $\mathrm{C}_{60}$ is much more stable against fusion and fragmentation compared with $\mathrm{C}_{30}$. This is particularly evident for the high temperature range from $2000 \mathrm{~K}$ to $3000 \mathrm{~K}$ which is relevant to the fullerene formation process. The high stability of the $\mathrm{C}_{60}$ molecule at high temperatures may be related to the high yield of $\mathrm{C}_{60}$ in the experimental formation process. However, a more 
(a)
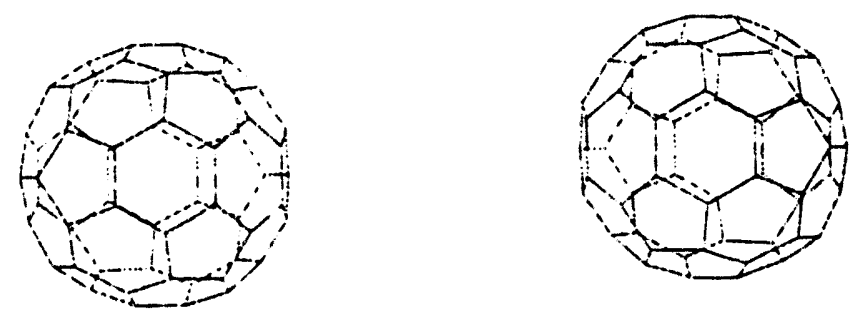

(b)

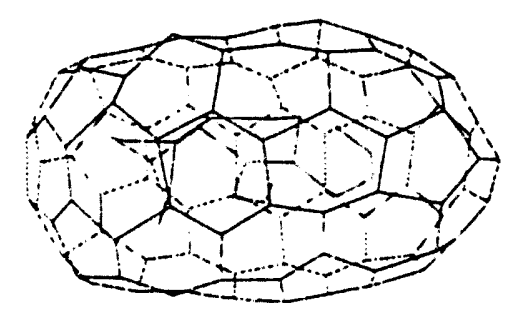

(c)

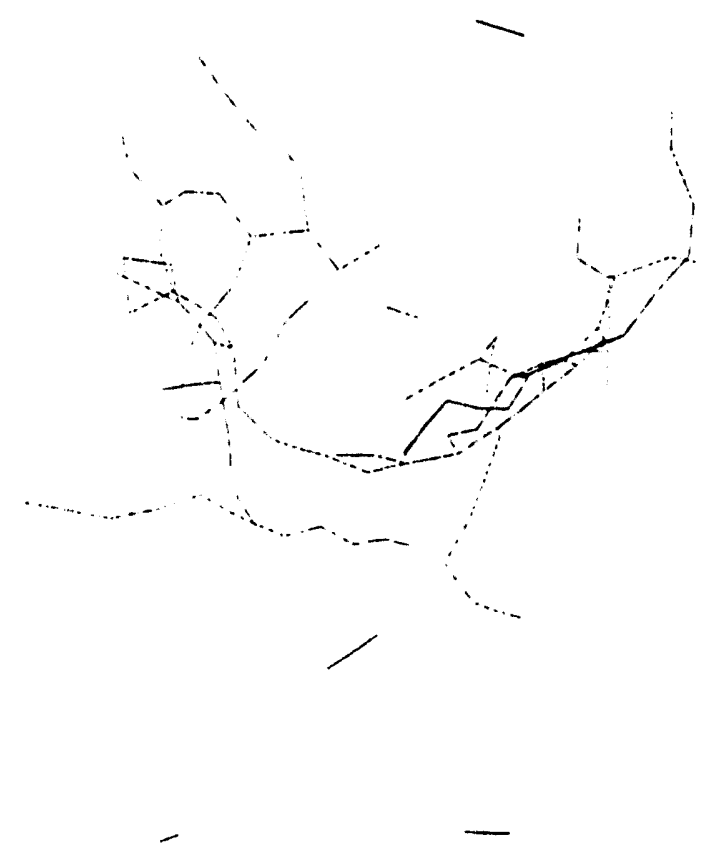

Figure 9.2: The schematic illustrations of three different collision stages for $\mathrm{C}_{60}$ (all these are sideviews of collision trajectories): (a) Bouncing (initial $\mathrm{T}=$ $0 \mathrm{~K}$, collision energy $=30 \mathrm{eV}$.) (b) Fusion, (initial $\mathrm{T}=2000 \mathrm{~K}$, collision energy $=72 \mathrm{eV}$.) (c) Fragmentation (initial $\mathrm{T}=0 \mathrm{~K}$, collision energy $=$ $300 \mathrm{eV}$.) 
detailed study of collisions between different carbon clusters and small carbon radicals is needed to make a final conclusion.

For the case of collisions of $\mathrm{C}_{60}$ molecules at $2000 \mathrm{~K}$, we made a detailed analysis of the energy transfer in the collision process as a function of collision energy in the low-energy regime. In this regime, when the molecules bounce back with their cage structures intact, it is easy to monitor the change in the translational kinetic energy of the system defined by

$$
\begin{gathered}
\Delta K=K_{c m}^{i n i t i a l}-K_{c m}^{\text {final }} \\
K_{c m}=N \frac{1}{2} m V_{c m}^{2}
\end{gathered}
$$

where $\mathrm{N}$ is the number of atoms for one molecule, $\mathrm{V}_{\mathrm{cm}}$ is the velocity of the center of mass of one of the molecules and $m$ is the mass of carbon atom. In Fig. 9.3(a) we plot the fractional decrease in the translation energy

$$
R_{t}=\frac{\Delta K}{K_{c m}^{\text {initial }}}
$$

as a function of the collision energy. We note that the $R_{t}$ stays fairly constant around $25 \%$ for low-energy collisions and increases as the collision energy approaches the critical fusion energy. The loss in translational energy $\Delta K$ is transferred to the internal degrees of freedom of the molecules. In the low-energy regime, the cage structures of the molecules remain intact, thus all of $\Delta K$ goes to heating up the outgoing molecules.

In Fig. 9.4, we plot the evolution of the translational kinetic energy, the vibrational kinetic energy and the potential energy of system, and the center-of-mass separation of the two molecules during the collision process for a typical collision in 
Table 9.1: The fusion energies of carbon clusters at different temperature and orientations. The collision energy is defined as total translational kinetic energy of system in the center of mass frame in the unit of $\mathrm{eV}$.

\begin{tabular}{|c|c|c|c|}
\hline & $\mathrm{T}=0 \mathrm{~K}$ & $\mathrm{~T}=2000 \mathrm{~K}$ & $\mathrm{~T}=3000 \mathrm{~K}$ \\
\hline $60(a)$ & 102 & \multirow{4}{*}{60} & \multirow{4}{*}{54} \\
\hline $60(b)$ & 84 & & \\
\hline $60(\mathrm{c})$ & 84 & & \\
\hline $60(\mathrm{~d})$ & 84 & & \\
\hline 30 & 51 & 30 & 9 \\
\hline
\end{tabular}

Table 9.2: The fragmentation energies of carbon clusters at different temperature and orientations. The collision energy is defined as total translational kinetic energy of system in the center of mass frame in the unit of $\mathrm{eV}$.

\begin{tabular}{|c|c|c|c|}
\hline & $\mathrm{T}=0 \mathrm{~K}$ & $\mathrm{~T}=2000 \mathrm{~K}$ & $\mathrm{~T}=3000 \mathrm{~K}$ \\
\hline $60(\mathrm{a})$ & 210 & \multirow{4}{*}{114} & \multirow{4}{*}{72} \\
\hline $60(\mathrm{~b})$ & 210 & & \\
\hline $60(\mathrm{c})$ & 204 & & \\
\hline $60(\mathrm{~d})$ & 198 & & \\
\hline 30 & 78 & 54 & 48 \\
\hline
\end{tabular}




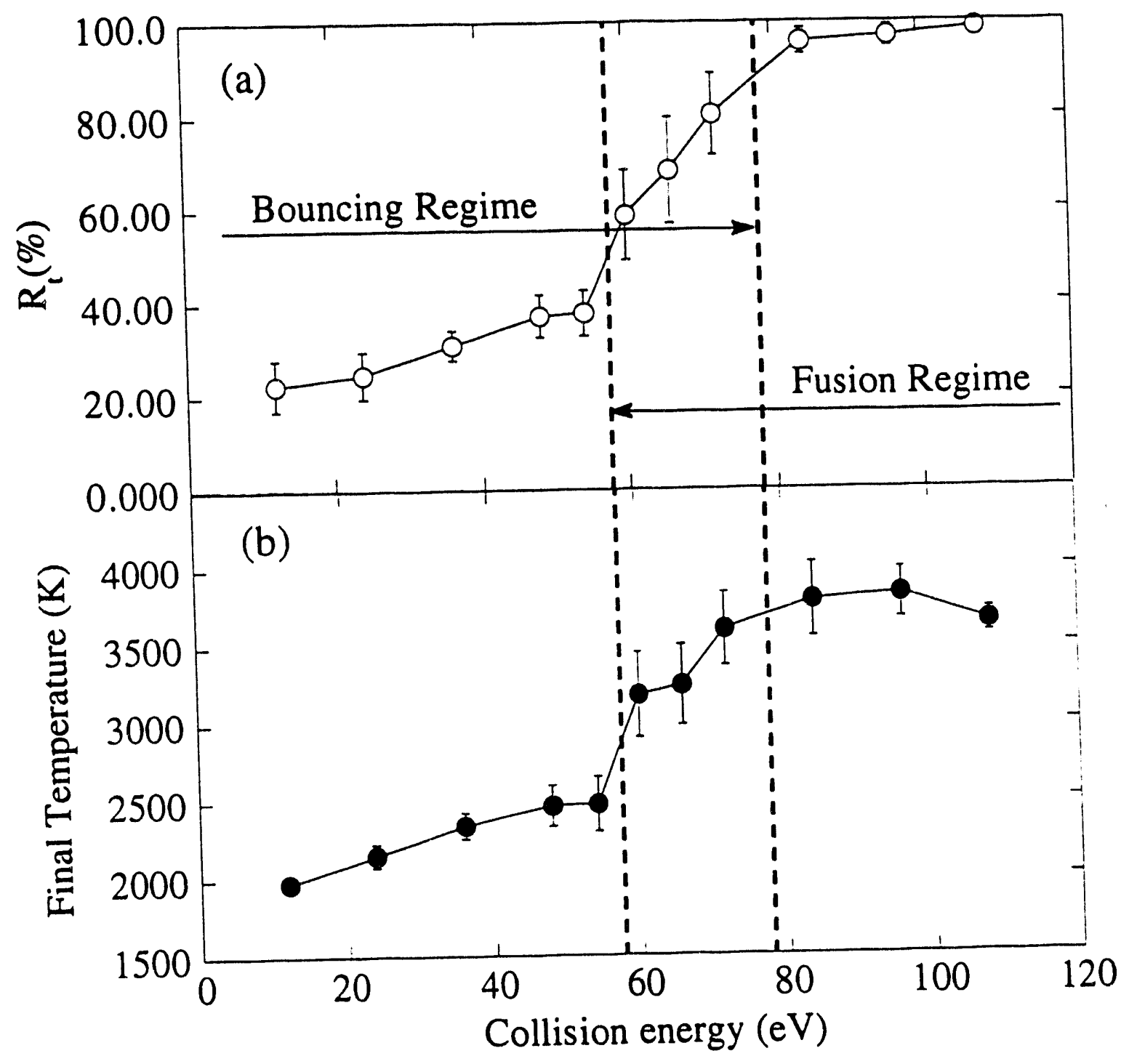

Figure 9.3: (a) The energy transfer ratio $R_{t}$ and (b) final temperature are plotted as the function of initial translational kinetic energy of center of mass for system. The error bars represent the corresponding standard deviations and the average initial temperature of clusters is $1930 \mathrm{~K}$. 
the low energy regime. The amount of vibrational kinetic energy in each molecule is obtained by

$$
K_{v i b}=\frac{1}{2} m \sum_{i}\left(\vec{v}_{i}-\vec{V}_{c m}\right)^{2}
$$

where the sum is over all atoms of a given molecule and $V_{\mathrm{cm}}$ is the center-of-mass velocity of the molecule.

In the fusion regime, all the translational kinetic energy is lost after collision, but not all of it is transformed into heat. Part of the energy is stored as chemical rearrangement energy in the new cluster. In Fig. 9.3(b), we plot the final temperature of the system after collision for both the bouncing and the fusion regime. The final temperature is obtained from the vibrational kinetic energy per degree of freedom. In the bouncing regime, the temperature of molecules after collision increases as the collision energy increases indicating that more and more heat is generated during the collision process. In the fusion regime, the final temperature of the cluster stays quite constant as the collision energy increases and even drops at the high-energy end of the fusion regime. The reason is that, as the collision energy increases, many bonds are broken in the collision process and most of the translational kinetic energy goes into potential energy to rearrange the cluster structure. In fact, when the collision is very close to the fragmentation regime, the final form of the cluster is no longer cage-like but becomes more like a sheet. This introduces a lot of dangling bonds which cannot be balanced by the increase in collision energy and the temperature of the cluster drops. For the plots in Fig. 9.3, the large error bars of these values in the transition regime are due to the occurrence of both types of processes, bouncing back and fusion. Among the trajectories for a given collision energy, the translational energy will be completely lost when two molecules stick together, but the molecules 
will still retain some of their translational kinetic energies when they bounce back. However $R_{t}$ is not $100 \%$, even though the two molecules have fused into one. This is because after the fusion, the groups of atoms from the colliding molecules have a collective mode of vibration with respect to the center of mass of the whole system, and the corresponding vibrational energy is treated as translational kinetic energy by Equation 9.2. This can be regarded as an artifact of the definition of $R_{t}$ (Equation 9.3). In the low-energy regime, the major factor is the impact parameter which will greatly affect the energy transfer and final temperature. A complete study with variable impact parameters is desired to make reasonable comparison with experimental results.

At zero temperature, we found that the outcomes depend not only on the collision energy but also on the orientations of the clusters. We found that buckyballs stick easier under bond-to-bond collision and the energy barrier is higher for face-to-face collisions. We also observed several interesting cases for ze-o-temperature collisions which are shown in the Fig. 9.5.

(a) "Russian-doll": In orientation A (pentagon to pentagon), the resultant configuration of the collision with $0.9 \mathrm{eV}$ per atom consists of one $\mathrm{C}_{20}$ cage trapped in a $\mathrm{C}_{100}$ cage (Fig. 9.5a). This kind of structure resembles the Bucky tube [53] with one carbon tube enclosed by another. Recently, truly 'Russian-doll' molecules, one fullerene enclosed in another fullerene, were also observed experimentally as a result of intense electron-beam irradiation [127]. Our simulations indicate another possibility that this kind of novel molecule may be obtained from collisions of buckyballs.

(b) "Peanut": With type-D orientation (hexagon to hexagon) and collision energy at $0.8 \mathrm{eV}$ per atom, two $\mathrm{C}_{60}$ will fuse and become a big cluster with 120 


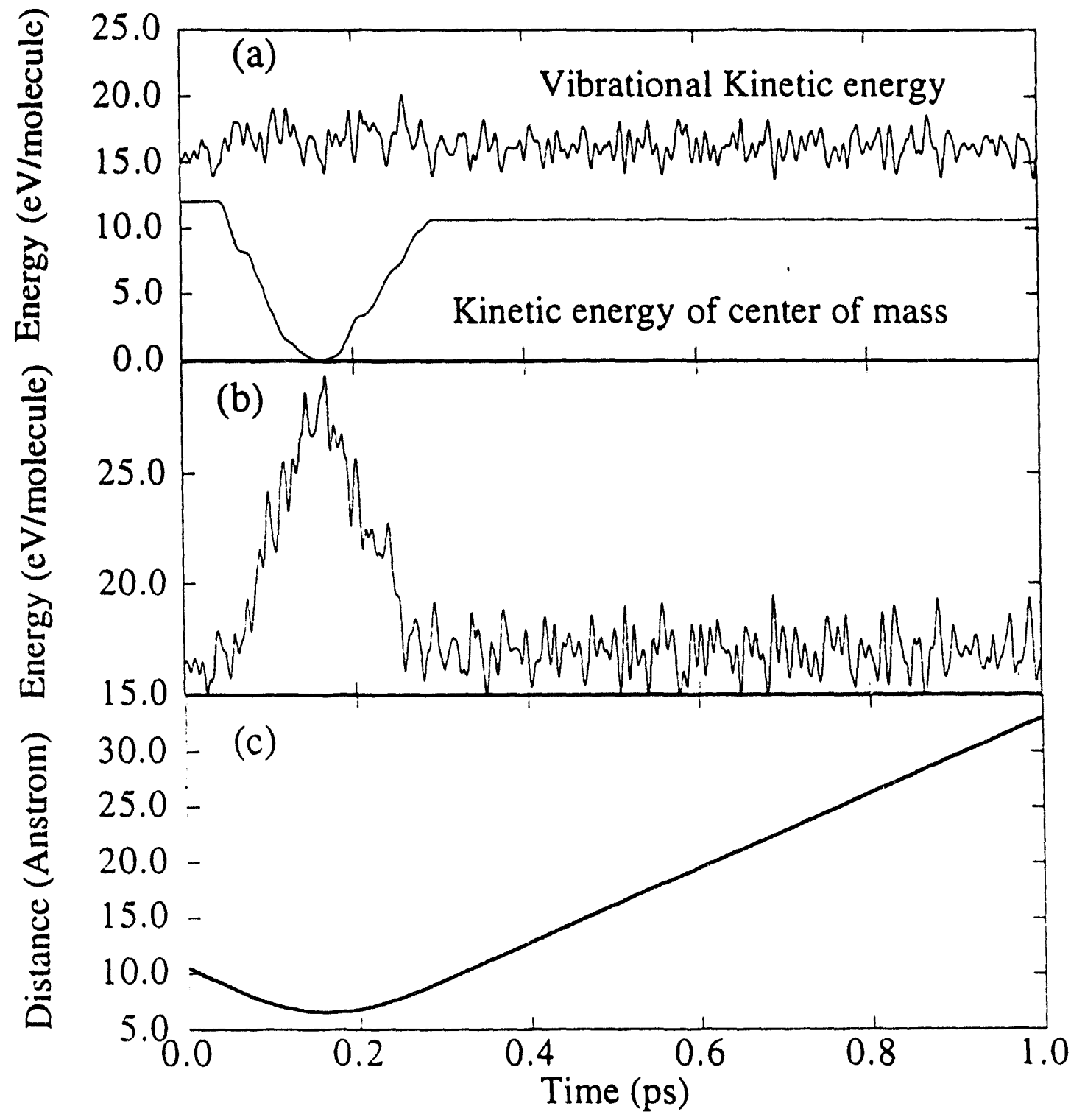

Figure 9.4: (a) The translational kinetic energy of center of mass, vibrational kinetic energy, (b) potential energy (relative to fully relaxed $\mathrm{C}_{60}$ ), and (c) the distance between the centers of mass of two buckyballs plotted as a function of time. The initial temperature of the clusters is $2000 \mathrm{~K}$ and the initial translational kinetic energy of the center of mass is $12 \mathrm{eV}$ per molecule. 
atoms in a peanut-like shape ( Fig. 9.5(c)). The neck part of this "peanut" contains six seven-member rings and consequently 6 extra pentagons (total 18 pentagons) to satisfy the Euler's theorem. These heptagons provide the negative curvature $[116,117,118,53,132]$ instead of the positive curvature provided by pentagons in fullerenes. However the binding energy of this resultant cage (after full relax) is far less than that of the spherical $\mathrm{C}_{120}$ isomers although it is more stable than two $\mathrm{C}_{60}$ cages by $2.6 \mathrm{eV}$.

(c) Baby cages: If we increase the collision energy to $1.0 \mathrm{eV}$ per atoms, the products of the collision are one $\mathrm{C}_{40}$ cage and two $\mathrm{C}_{40}$ caps (Fig. 9.5b). The baby cage coming out of a collision is the ground-state isomer for $\mathrm{C}_{40}$ (see Fig. 6.1). The two $\mathrm{C}_{40}$ caps have dangling bonds at the edges and they will easily stick with other carbon fragments.

While these special orientation collisions are rare in the gas phase, because they require specific alignments of the molecules, there are possibilities that they can be achieved in the low temperature solid phase. For $\mathrm{C}_{60}$ solid, there is an orientational phase transition around $250 \mathrm{~K}$ [133]. At room temperature, pure solid $\mathrm{C}_{60}$ is in a $f c c$ structure and each buckyball can spin freely. But at low temperature or with doping, the rotations are suppressed and the orientation of each ball is pinned. Then we can apply a sudden compression in a preferred direction to produce collisions between buckyballs with a fixed impact orientation. The results of our simulation provide valuable informations to choose these specific orientations and the collision energies necessary to obtain these novel molecules. 
(a)

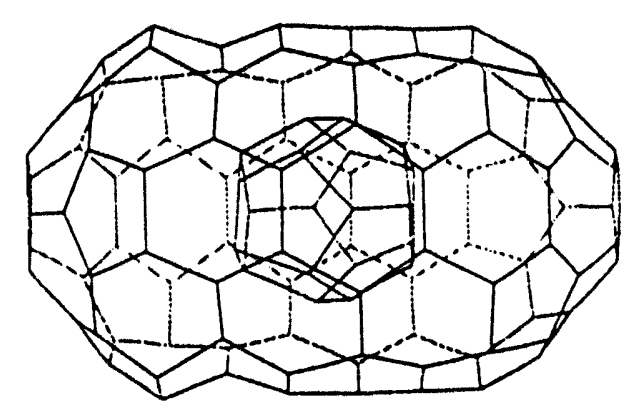

(b)

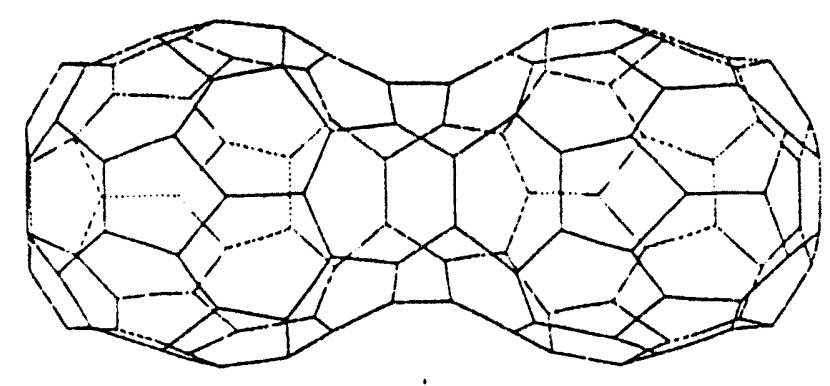

(c)
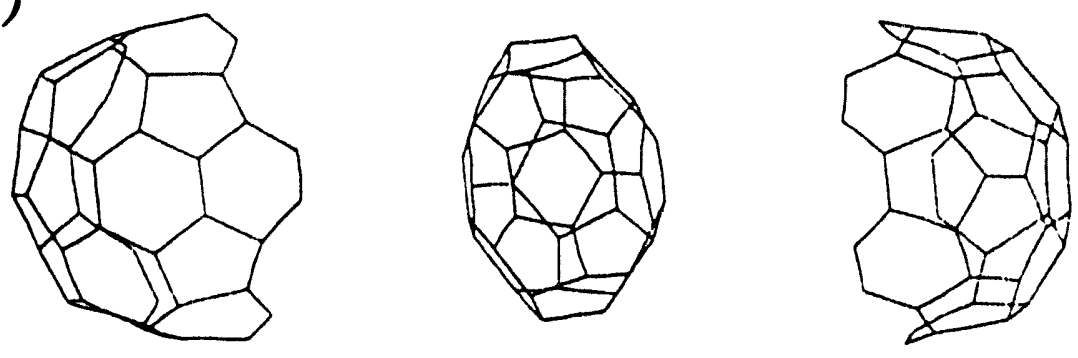

Figure 9.5: The line draws of the resultant typical molecules produced by collisions between two $\mathrm{C}_{60}$ : (a) "Russian Doll", (b) "peanut" and (c) "baby-cage". (all these are the sideviews of collision trajectories). 


\section{Summary}

In the clustering process and thermal equilibrium, collisions between clusters play a very important role and it may be one of the key process for understanding the abundance of various clusters. We have simulated the collision between $\mathrm{C}_{60}$ molecules by tight-binding molecular-dynamics. We found that the clusters will bounce back at low energies, stick together at higher energies and break up at very high collision energies. The most possible channel for the depletion of small clusters at high densities is sticking. Since the newly formed clusters have dangling bonds, they are more likely to stick with other clusters and accumulate to become soot. As the temperature goes higher, the clusters stick more easily. The buckyball $\mathrm{C}_{60}$ molecule is found to have much higher survival energy than that of $\mathrm{C}_{30}$. A complete picture requires further investigations of collisions between clusters and small carbon fragments in the gas phase. Also, in reality, most collisions are not head-on collisions so experiments will obtain higher critical energies for fusion and fragmentation than the numbers reported here. Under certain collision energies and orientations, we also observe several very interesting novel molecules coming out of the collisions. These may provide some useful clues for the synthesis of novel molecules by sudden directional compression of solid $\mathrm{C}_{60}$ at low temperature. 


\section{BIBLIOGRAPHY}

(1) J. C. Jamieson, Science 140, 72 (1963).

[2] B. S. Hickman, J. Mater. Sci. 4, 554 (1969).

[3] D. T. Keating and S. J. LaPlaca, J. Phys. Chem. Solids, 35, 879 (1974).

[4] S. C. Moss, D. T. Keating, and J. D. Axe, in Phase Transitions, edited by L. E. Cross (Pergamon, New York, 1973), p.179.

[5] J. D. Axe, D. T. Keating, and S. C. Moss, Phys. Rew. Lett. 35, 530 (1975).

[6] J. M. Sanchez and D. De Fontaine, Phys. Rev. Lett. 35, 227 (1975).

[7] W. Lin, H. Spalt, and B. W. Batterman, Phys. Rev. B 13, 5158 (1976).

[8] Y. Noda, Y. Yamada, and S. M. Shapiro, Phys. Rev. B 40, 5995 (1989).

[9] K. M. Ho, C. L. Fu, and B. N. Harmon, Phys. Rev. B. 29, 1575 (1984).

[10] P.-A. Lindgard and O. G. Mouritsen, Phys. Rev. Lett. 5158 (1976).

[11] Y. Noda, Y. Yamada, and S. M. Shapiro, Phys. Rev. B 40, 5995 (1989).

[12] P.-A. Lindgard and O. G. Mouritsen, Phys. Rev. Lett. 57, 2458 (1986).

[13] Y. Y. Ye, Y. Chen, K. M. Ho, B. N. Harmon, and P. A. Lindgard, Phys. Rew. Lett. 58, 1769 (1987).

[14] V. P. Dmitriev, S. B. Rochal, Yu. M. Gufa, and P. Toledano, Phys. Rev. Lett. 60, 1958 (1988).

[15] J. A. Krumhansl and R. J. Gooding, Phys. Rev. B 34, 3047 (1989). 
(16) F. Willaime and C. Massobrio, Phys. Rev. Lett. 63, 2:44 (1989), Phys. Rev. B 43, 11653 (1991).

[17] Y. Yamada and K. Fuchizaki, Phys. Rev. B 42, 9420 (1990).

[18] C. Stassis, J. Zarestky, and N. Wakabayashi, Phys. Rev. Lett. 41, 1726 (1978);

C. Stassis and J. Zarestky, Solid State Commun. 52, 7 (1984).

(19) A. Heiming, W. Petry, J. Trampenau, M. Alba, and C. Herzig, and G. Vogl, Phys. Rev. B. 40, 11425 (1989).

[20] A. Heiming, W. Petry, J. Trampenau, M. Alba, C. Herzig, H. R. Schober, and G. Vogl, Phys. Rev. B. 43, 10948 (1991).

(21) F. Guthoff, W, Petry, C. Stassis, A. Heiming, B. Hennion, C. Herzig, and J. Trampenau, Phys. Rev. B 47, 2563 (1993).

[22] W. Petry, T. Flottmann, A. Heiming, J. Trampenau, M. Alba, and G. Vogl, Phys. Rev. B 61, 722 (1988).

[23] M. S. Daw and M. I. Baskes, Phys. Rev. Lett. 50, $128 j$ (1983); M. S. Daw and M. I. Baskes, Phys. Rev. B. 29, 6443 (1984).

(24) M. S. Daw and S. M. Foiles, J. Vac. Sci. Technol. A 4, 1412 (1986), M. S. Daw, Surf. Sci. 166, L161 (1986).

[25] S. M. Foiles, Phys. Rev. B 32, 3409 (1985).

[26] M. S. Daw and R. L. Hatcher, Solid State Commun. 56, 697 (1985), J. S. Nelson, E. C. Sowa, and M. S. Daw, Phys. Rev. Lett. 61, 1977 (1988).

[27) S. M. Foiles and M. I. Baskes, and M. S. Daw, Phys. Rev. B 33, 7983 (1986).

[28] J. Belak and I. F. Stowers, NATO ASI Proceedings, Fundamentals of Friction, edited by I. L. Singer and H. M. Pollock (Kluwer, Dordrecht, 1992) p. 511.

[29] E. A. Rohlfing, D. M. Cox, A. J. Kaldor, J. Chem. Phys. 81, 3322 (1984).

[30] R. E. Smalley, Acc. Chem. Res. 25, 98 (1992).

[31] H. W. Kroto, J. R. Heath, S. C. O'Brien, R. F. Curl, and R. E. Smalley, Nature (London) 318, 162 (1985).

[32] W. Kratschmer, L. D. Lamb, K. Fostiropoulos, and D. R. Hoffman, Nature (London) 347, 354 (1990). 
[33] R. Taylor, J. P. Hare, A. K. Abdule-Sada, and H. W. Kroto, J. Chem. Soc., Chem. Commun., 20, 1423 (1990).

(34) W. Kratchmer, K. Fostiropoulos, and D. R. Huffman, Chem. Phys. Lett. 170, 167 (1990).

[35] A. F. Hebard, M. J. Rosseinsky, R. C. Haddon, D. W. Murphy, S. H. Glarum, T. T. M. Palstra, A. P. Ramirez, and A. R. Kortan, Nature (London) 350, 600 (1991).

[36] P. M. Allemand, K. C. Khemani, A. Koch, F. Wudl, K. Holczer, S. Donovcm, G. Gruner, and J. D. Thompson, Science 253, 301 (1991).

[37] Y. Chai, T. Guo, C. Jin, R. E. Smalley, J. Phys. Chem. 95, 7564 (1991).

[38] P. N. Keating, Phys. Rev. 145, 637 (1966).

[39] J. Tersoff, Phys. Rev. Lett. 61, 2879 (1988).

[40] C. H. Xu, C. Z. Wang, C. T. Chan, and K. M. Ho, Condens. Matter, 4, 6047 (1992).

[41] F. Diederich, R. Ettl, Y. Rubin, R. L. Whetten, R. Beck, M. M. Alvarez, S. Anz, D. Sensharma, F. Wudl, K. C. Khemani, A. Koch, Science 252, 548 (1991).

[42] R. Ettl, I. Chao, F. Diederich, and R. L. Whetten, Nature 353, 149 (1991).

[43] F. Diederich, R. L. Whetten, C. Thilgen, R. Ettl, I. Chao, M. M. Alvarez, Science 254, 1768 (1991).

[44] K. Kikuchi, N. Nakahara, T. Wakabayashi, M. Honda, H. Matsumiya, T. Moriwaki, S. Suzuki, H. Shiromaru, K. Saito, K. Yamauchi, I. Ikemoto, and Y. Achiba, Chem. Phys. Lett. 188, 177 (1992).

(45] K. Kikuchi, N. Nakahara, T. Wakabayashi, S. Suzuki, H. Shiromaru, Y. Miyake, K. Saito, I Ikemoto, M. Kainosho, Y. Achiba, Nature 357, 142 (1992).

[46] B. L. Zhang, C. Z. Wang, K. M. Ho, Chem. Phys. Lett. 193, 225 (1992).

[47] B. L. Zhang, C. H. Xu, C. Z. Wang, C. T. Chan, and K. M. Ho, Phys. Rev. B 46, 7333 (1992).

[48] B. L. Zhang, C. Z. Wang, K. M. Ho, C. H. Xu, and C. T. Chan, J. Chem. Phys. 97, 5007 (1992). 
[49] B. L. Zhang, C. Z. Wang, K. M. Ho, C. H. Xu, and C. T. Chan, J. Chem. Phys. 98, 3095 (1993).

[50] B. L. Zhang, C. Z. Wang, and K. M. Ho, Phys. Rev. B, 47, 1643 (1992).

[51] B. L. Zhang, C. Z. Wang, C. T. Chan, and K. M. Ho, Phys. Rev. B, (submitted).

[52] B. L. Zhang, C. Z, Wang, C. T. Chan, and K. M. Ho, J. Phys. Chem. 97, 3134 (1993).

[53] S. Iijima, Nature (London) 354, 56 (1991), and S. Iijima, Nature (London) 356, 776 (1992).

[54] A. L. Mackay and H. Terrones, Nature (London) 352, 762 (1991).

[55] D. Vanderbilt and J. Tersoff, Phys. Rev. lett. 68, 511 (1992).

[56] T. Lenosky, X. Gonze, M. Teter, and V. Elser, Nature (London) 355, 333 (1992).

[57] B. L. Zhang, C. T. Chan, C. Z. Wang, and K. M. Ho. (unpublished).

[58] E. Clementi and C. Roetti, Atomic Data and Nuclear Data Tables (Academic, New York, 1974), Vol. 14. Nos. 3 and 4.

[59] E. S. Fisher and C. J. Renken, J. Nucl. Mater. 4, 311 (1961).

[60] S. H. Liu, C. Stassis, and K. M. Ho, Phys. Rev. B 24, 5093 (1981).

[61] C. Stassis, J. Zaretsky, D. Arch, O. D. McMaster, and B. N. Harmon, Phys. Rev. B. 18, 2632 (1978)

[62] C. W. Gear, The numerical integration of ordinary differential equations of various orders, Report ANL 7126, Argonne National Laboratory, (1966); C. W. Gear, Numerical initial value problems in ordinary differential equations, Prentice-Hall, Englewood Cliffs, NJ (1971).

[63] A. Nordsiek, Math. Comput. 16, 22 (1962).

[64] L. V. Woodcock, Chem. Phys. Lett. 10, 257 (1971).

[65] H. C. Anderson, J. Chem. Phys. 72, 2384 (1980).

[66] W. G. Hoover, A. J. C. Ladd, and B. Moran, Phys. Rev. Lett. 48, 1818 (1982); D. J. Evans, J. Chem. Phys. 78, 3297 (1983). 
67] J. M. Haile and S. Gupta, J. Chem. Phys. 79, 3067 (1983).

[68] S. Nosé, J. Chem. Phys. 81, 511 (1984).

69] S. Nosé, Molec. Phys. 52, 255 (1984).

[70] M. Parrinello and A. Rahman, J. Appl. Phys. 52, 7182 (1981).

[71] J. Goldak, L. T. Lloyd, and C. S. Barrett, Phys. Rev. 144, 478 (1966).

[72] J. M. Holender, Phys. Rev. B 41, 8054 (1990).

[73] S. R. Philpot, J. F. Lutske, D. Wolf, and S. Yip, Phys. Rev. B 40, 2831 (1989).

[74] W. G. Burgers, Physica 1, 561 (1934).

[75] N. W. Ashcroft, N. D. Mermin, Solid State Physics (Holt, Rinehart and Winston, New York 1976), p.792.

76] N. Boccara and G. Sarma, Physics 1, 219 (1965).

(77) J. R. Morris and R. J. Gooding, Phys. Rev. B 43,

[78] R. E. Watson and M. Weinert, Phys. Rev. B 30, 1641 (1984).

(79) D. E. Turner (to be published).

[80] C. Stassis and W. Petry, (private communication).

[81] D. J. Chadi, Phys. Rev. Lett. 41, 1062 (1978); Phys. Rev. B 29, 785 (1984); Phys. Rev. Lett. 59, 1691 (1988).

[82] W. A. Harrison, Electronic Structure and Properties of Solids, W. H. Freeman and Company, San Francisco (1980).

83 D. Tomanek and M. A. Schluter, Phys. Rev. Lett. 56. 1055 (1986); Phys. Rev. B 36, 1208 (1987).

84 D. G. Allen and E. J. Mele, Phys. Rev. Lett. 53, 826 (1984); O. L. Alerhand, D. G. Allen, and E. J. Mele, Phys. Rev. Lett.

55, 2700 (1985).

85 F. S. Khan and J. Q. Broughton, Phys. Rev. B 39, 3688 (1989).

86 M. Menon and R. E. Allen, Phys. Rev. B 33, 7099 (1986); Phys. Rev. B 38, 6196 (1988). 
[87] K. Raghavachari and J. S. Binkley, J. Chem. Phys. 87, 2191 (1987).

[88] J. L. Martin, (private communication)

[89] G. Fernando, G.-X. Qian, M. Winert, and J. D. Davenport, Phys. Rev. B 40, 7985 (1989); G.-X. Qian, M. Weinert, G. Fernando, and J. D. Davenport, Phys. Rev. Lett. 64, 1146 (1990).

[90] M. Pederson and K. Jackson, Phys. Rev. B 43, 7312 (1991).

[91] R. M. Wentzcovitch, J. L. Martin, and P. B. Allen, Phys. Rev. B 45, 11372 (1992).

[92] N. D. Mermin, Phys. Rev. 137, A1441 (1965).

[93] P. W. Fowler, J. E. Cremona and J. I. Steer, Theor. Chim. Acta 73, 1 (1988).

[94] T. G. Schmalz, W. A. Seitz, D. J. Klein and G. E. Hite, J. Am. Chem. Soc. 110,1113 (1988).

[95] D. Bakowies and W. Thiel, J. Am. Chem. Soc. 113, 3704 (1991).

[96] D. E. Manolopoulos, J. C. May and S. E. Down, Chem. Phys. Lett. 181, 105 (1991).

[97] P. W. Fowler, J. Chem. Soc. Faraday Commun. 87, 1945 (1991).

[98] D. E. Manolopoulos, J. Chem. Soc. Faraday Commun. 87, 2861 (1991).

[99] P. W. Fowler, R. C. Batten and D. E. Manolopoulos, J. Chem. Soc. Faraday Commun. 87, 3103 (1991).

[100] D. E. Manolopoulos, P. W. Fowler, J. Chem. Phys. 96, 7603 (1992).

[101] J. R. Chelikowsky, Phys. Rev. Lett. 67, 2970 (1991).

[102] C. Z. Wang, C. H. Xu, C. T. Chan, and K. M. Ho, Phys. Rev. B

[103] A. J. Stone and D. J. Wales, Chem. Phys. Lett. 99, 4899 (1977).

[104] R. W. Hasse and V. V. Avilov, Phys. Rev. A, 44, 4506 (1991).

[105] J. B. Weinrach, K. L. Carter, D. W. Bennett, and H. K. McDowell, J. Chem. Ed., 67, 995 (1990).

[106] H. W. Kroto, Nature 329, 529 (1987). 
[107] C. Kittel, Introduction io Solid State Physics, (John Wiley \& Sons, New York, 1986), p 205.

[108] X. Q. Wang, C. Z. Wang, B. L. Zhang, and K. M. Ho. Phys. Rev. Lett. 69, 69 (1992).

[109] T. Wakabayashi and Y. Achiba, Chem. Phys. Lett. 190, 465 (1992).

[110] X. Q. Wang, C. Z. Wang, B. L. Zhang, and K. M. Ho. Chem. Phys. Lett. 200, 26 (1992).

[111] K. Raghavachari, Chem. Phys. Lett. 190, 397 (1992).

[112] X. Q. Wang, C. Z. Wang, B. L. Zhang, and K. M. Ho, Chem. Phys. Lett. to be published.

[113] Y. Achiba, (private communication).

[114] S. C. O'Brien, J. R. Heath, R. F. Curl, and R. E. Smalley, J. Chem. Phys. 88, 220 (1988).

[115] J. R. Heath Fullerenes-Synthesis, Properties, and Chemistry of Large Carbon Clusters; G. S. Hammond and V. J. Kuck Eds.; ACS Symposium Series 481; American Chemical Society, Washington, DC, 1992 Chapter 1, pp 1-21.

[116] A. L. Mackay and H. Terrones, Nature (London) 352, 762 (1991).

(117) D. Vanderbilt and J. Tersoff, Phys. Rev. Lett. 68, 511 (1992).

[118] T. Lenosky, X. Gonze, M. Teter, and V. Elser, Nature (London) 355, 333 (1992).

[119] R. Phillips, D. A. Drabold, T. Lenosky, G. B. Adams, and O. F. Sankey, Phys. Rev. E 1941 (1992).

[120] S. J. Townsend, T. J. Lenosky, D. A. Muller, C. S. Nichols, and V. Elser, Phy. Rev. Lett. 69, 921 (1992).

[121] W. A. Kamitakahara, (private communication).

[122| C. Z. Wang (to be published).

[123] S. C. O'Brien, J. R. Heath, R. F. Curl, and R. E. Smalley, J. Chem. Phys. 88, 220 (1988). 
[124] S. W. McElvany, M. M. Ross, J. H. Callahan, Mater. Res. Soc. Proc. 206, 697 (1991).

[125] R. D. Beck, S. J. Pamela, M. M. Alvarez, F. Diederich, and R. L. Whetten, J. Phys. Chem. 95, 8402 (1991).

[126] H. G. Busman, T. Lill, I. V. Hertel, Chem. Phys. Lett. 187, 459 (1991).

[127] D. Ugarte, Nature (London) 359, 707 (1992)

[128] E. E. B. Campbell, A. Hielscher, R. Ehlich, V. Schyja, and I. V. Hertel, Nuclear Physics Concepts in the Study Atomic Cluster Physics, Lecture Notes in Physics, (Springer, 1992) Vol. 404, p 185.

[129] R. C. Mowrey, D. W. Brenner, B. I. Dunlap, J. W. Mintmire, and C. T. White, J. Phys. Chem. 95, 7138 (1991).

[130] P. Ballone and P. Milani. Z. Phys. D, 19, 439 (1991).

[131] G. Seifert and R. Schmidt, (to be published).

[132] B. I. Dunlap, J. Phys. Chem. 95, 5763 (1991).

[133] P. A. Heiney, J. E. Fischer, A. R. McGhie, W. J. Romanow, A. M. Denenstein, J. McCauley Jr., A. B. Smith III, and D. E. Cox, Phys. Rev. Lett. 66, 2911 (1991). 
